\title{
History of research on C. elegans and other free-living nematodes as model organisms
}

\author{
Victor Marc Nigon ${ }^{1}$ and Marie-Anne Félix ${ }^{2 \mathcal{S}}$ \\ ${ }^{1}$ Université de Lyon 1, France \\ ${ }^{2}$ Ecole Normale Supérieure, Paris, France
}

\section{Table of Contents}

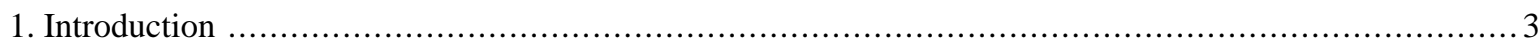

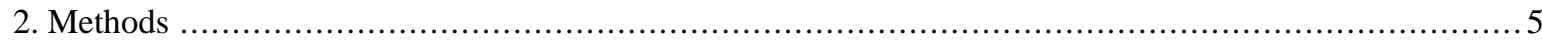

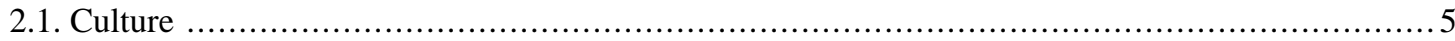

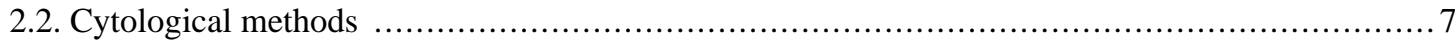

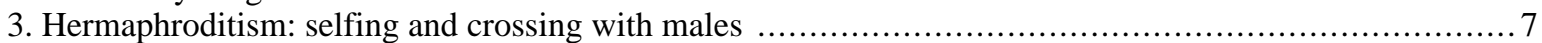

3.1. Protandric hermaphroditism as a modification of the female ..................................... 8

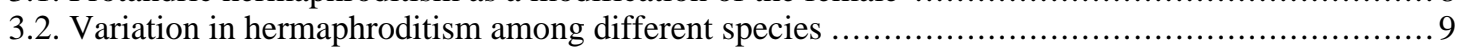

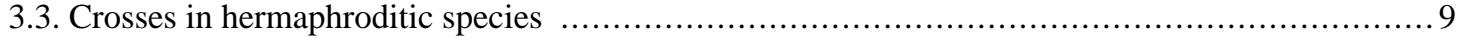

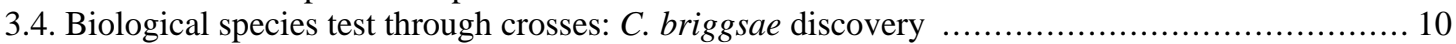

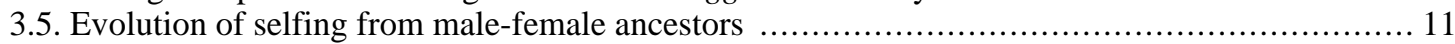

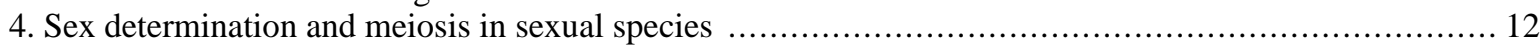

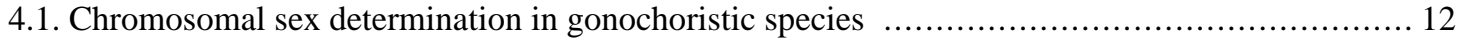

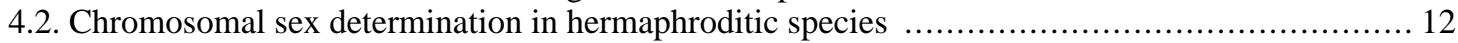

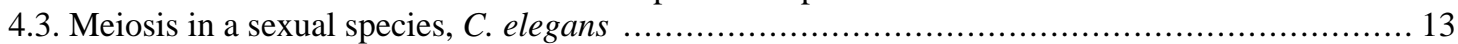

4.4. The X-chromosome-to-autosome ratio in sex determination: use of a tetraploid line ............. 15

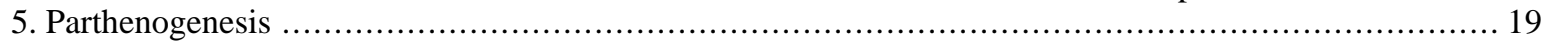

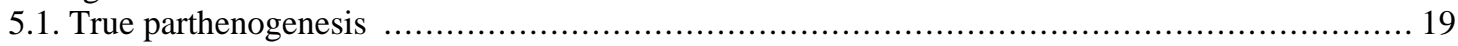

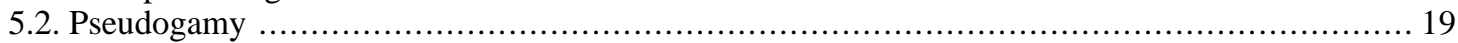

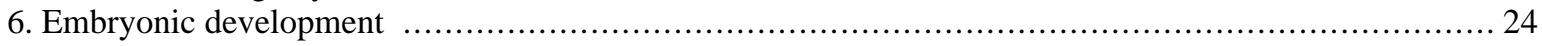

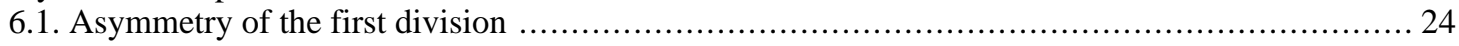

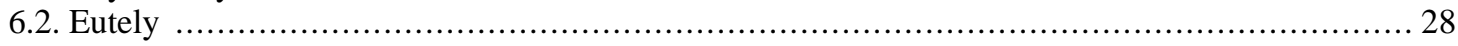

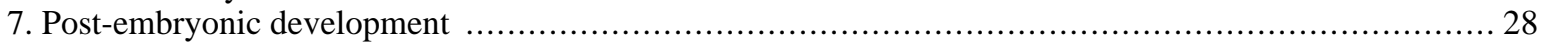

\footnotetext{
*Edited by Paul W. Sternberg. Last revised October 11, 2015. Published in its final form September 07, 2017. This chapter should be cited as: Nigon, V.M., and Félix, M.-A. History of research on C. elegans and other free-living nematodes as model organisms. (September, 07, 2017), WormBook, ed. The C. elegans Research Community, WormBook, doi/10.1895/wormbook.1.181.1, http://www.wormbook.org.

Copyright: () 2017 Victor Marc Nigon and Marie-Anne Félix. This is an open-access article distributed under the terms of the Creative Commons Attribution License, which permits unrestricted use, distribution, and reproduction in any medium, provided the original author and source are credited.

${ }^{\S}$ To whom correspondence should be addressed.Email: felix@biologie.ens.fr
} 


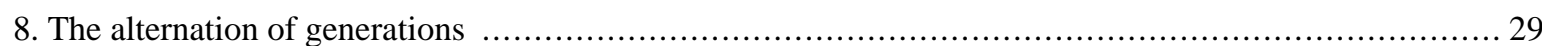

8.1. Gonochoristic free-living and hermaphroditic parasitic generations: Rhabdias bufonis .............. 30

8.2. Gonochoristic free-living and parthenogenetic parasitic generations: Strongyloides ratti ........... 30

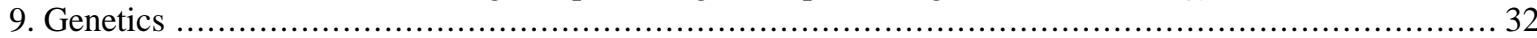

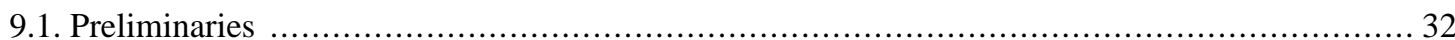

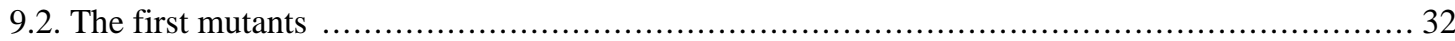

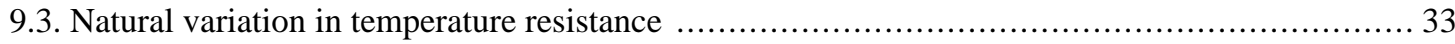

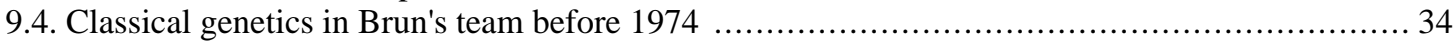

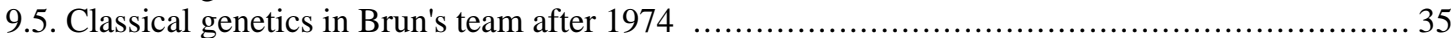

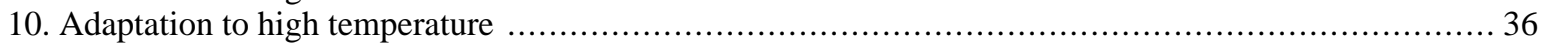

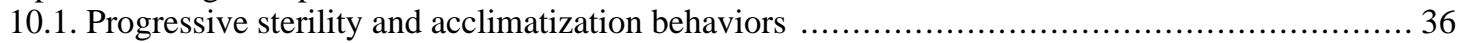

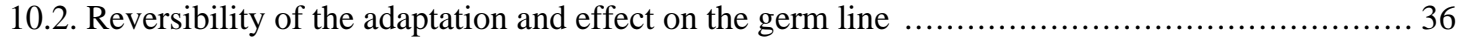

10.3. Hypotheses of non-genic factors and induction by high temperature .............................. 37

11. Effect of parental aging on fertility of the next generation .............................................. 38

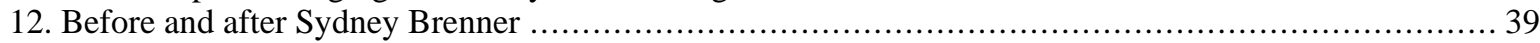

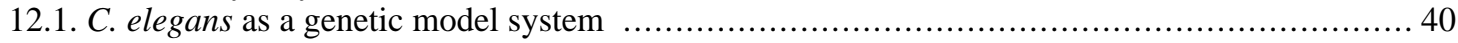

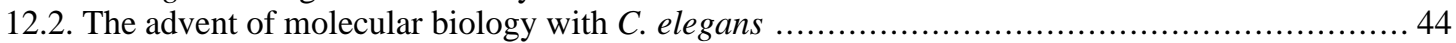

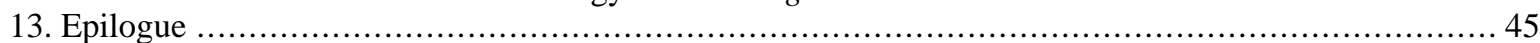

13.1. At the core of genetics: reproduction, sex determination, meiosis, and polyploidy ................. 46

13.2. Progressive multigenerational sterility and acclimatization ......................................... 47

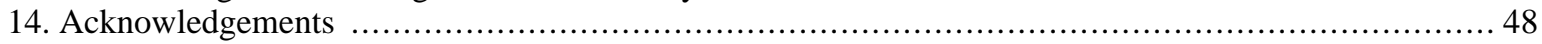

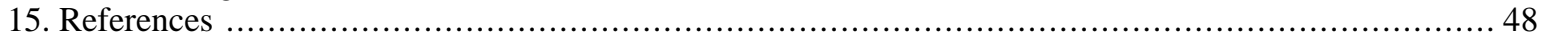

16. Appendix 1. Table 3. Main species mentioned in the text with their modern names and mode of

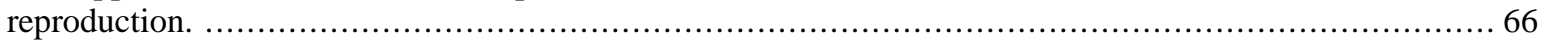

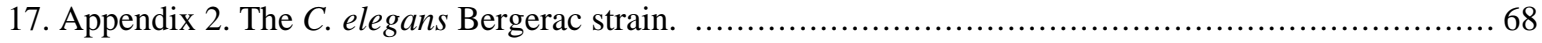

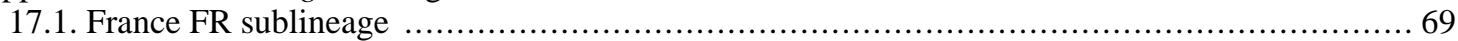

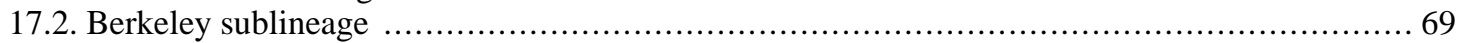

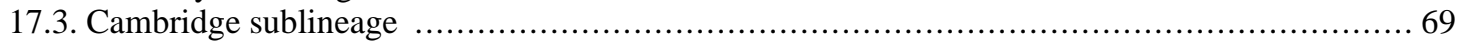

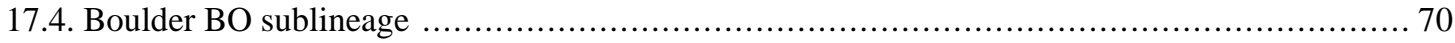

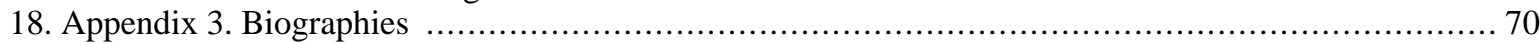

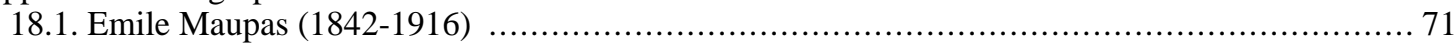

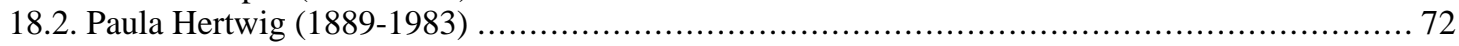

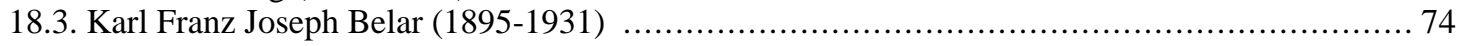

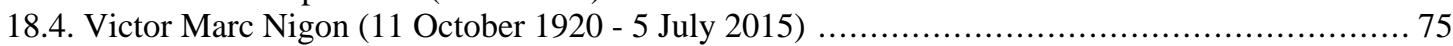

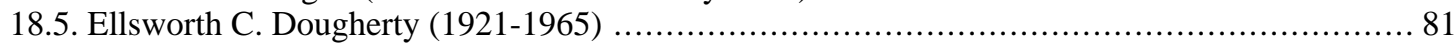

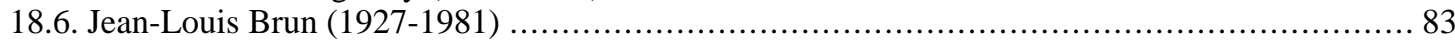

\begin{abstract}
The nematode Caenorhabditis elegans is now a major model organism in biology. The choice of Sydney Brenner to adopt this species in the mid-1960s and the success of his team in raising it to a model organism status have been told (http://www.wormbook.org/toc_wormhistory.html; Brenner, 2001; Ankeny, 2001). Here we review the pre-Brenner history of the use of free-living nematodes as models for general questions in biology. We focus on the period that started in 1899 with the first publication of Emile Maupas mentioning Rhabditis elegans and ended in 1974 with the first publications by Brenner. A common thread in this period, aided by the variety in modes of reproduction of different nematode species, is found in studies of meiosis, fertilization, heredity, and sex determination. Maupas in his 1900 opus on reproduction had already chosen C. elegans as the species of reference. Hikokura Honda determined its hermaphrodite chromosomal content in 1925. C. elegans was again isolated and chosen as a main subject by Victor Nigon in the 1940-50s. Nigon mastered crosses between $C$. elegans hermaphrodites and males, described the meiotic behavior of chromosomes in XX hermaphrodites and XO males and, using tetraploids, correctly inferred that seX was determined by $\mathrm{X}$ chromosome to autosome dosage. With Ellsworth Dougherty, Nigon isolated and studied a $C$. briggsae body size mutant and a $C$. elegans slow growth mutant. Dougherty and his team devoted most of their work to finding a defined culture medium to screen for physiological mutants, focusing on C. briggsae. With Helene Fatt, Dougherty also performed the first genetic study of natural variation in $C$. elegans, concerning the difference in heat resistance of the Bergerac and Bristol strains. Jean Brun, a student of Nigon, performed a long and remarkable experiment in acclimatization of $C$. elegans Bergerac to higher temperatures, the significance of which remains to be clarified.
\end{abstract}




\section{Introduction}

The small nematode roundworm Caenorhabditis elegans has become one of the main model organisms, allowing for major discoveries in molecular and cell biology, on par with the vinegar fly (Drosophila melanogaster) and baker's yeast (Saccharomyces cerevisiae). Historical studies of the development of C. elegans as a model organism usually start with the major figure of Sydney Brenner in the 1960s and neglect the earlier period. We were led to write about this early history for a number of reasons. First, this account helps bring to light historical developments in the study of free-living nematodes as model organisms, with their difficulties, stimulations, achievements, and cross-influences. Second, this work will also hopefully revive scientific questions that were forgotten or neglected over the last 50 years, which, in the light of today's knowledge, may lead to new research avenues. Finally, many of the early studies were published in French or German and are therefore not easily read by modern readers. Note that the first author of this chapter worked on C. elegans and other free-living nematodes in the 1940-50s, and particular emphasis is thus given to his personal account and biography (see Appendix 3, Section 18.4).

This article will focus on free-living nematodes, with particular attention to C. elegans. Yet, of note, parasitic nematodes such as Ascaris have played a key role in the history of biology. Indeed, in the period spanning 1880-1910, Ascaris was arguably the first model organism. The species A. megalocephala (now called Parascaris equorum) was then commonly found in horses, A. suum and A. lumbricoides in pigs and humans. As stated by Edouard van Beneden, "I have found with the Ascaris of the horse, a wonderful material. I am convinced that the egg of this nematode will soon become a classical object of study to investigate and illustrate the phenomena connected with fecundation." (Van Beneden, 1883; Hamoir, 1992). Gametogenesis and fertilization could be observed readily on fixed and stained material because of the transparency of reproductive organs, the large sperm size, and the small number of chromosomes (one pair in the univalens variety of the horse parasite, two in the bivalens variety). Cells, chromosomes, and centrosomes could be followed during meiosis, fertilization, and early development. Ascaris species thereby greatly contributed to the discovery of basic biological mechanisms, such as fertilization and meiosis (Van Beneden, 1883; Boveri, 1887; reviewed in Hamoir, 1992; Maderspacher, 2008), and later to studies in development, including the role of nucleus-cytoplasm interactions, embryonic axis formation, germ cell lineage (Boveri, 1892; Boveri, 1899: Boveri, 1910; zur Strassen, 1896; zur Strassen, 1959; Spemann, 1895; Muller, 1903; Bonfig, 1925), and the nervous system (Goldschmidt, 1908; Goldschmidt, 1909). Two features of Ascaris turned out to be somewhat idiosyncratic. In particular, chromatin diminution (Müller, 1996) only occurs in some nematodes such as Ascaris, but not in C. elegans. The elimination of chromosome fragments in somatic cells thus did not turn out to be a general mechanism for divergence of cell fates during development. The second feature, invariance of cell number (Martini, 1923), is more commonly found in nematodes and other organisms. The reproducibility of nematode cell lineages turned out to be of utmost practical importance in post-1974 studies of apoptosis, development, and neurobiology in C. elegans.

The success of parasitic nematode species as biological models thus set the stage for free-living nematodes, "the small nematodes" as they were called, to be adopted by a few biologists, who albeit were often not in the mainstream of research. Free-living species, in contrast to obligate parasites, can be cultured in the absence of a host through their whole lifecycle, which is one of the characteristics that has allowed C. elegans to become a major model species for genetic analysis.

The first important phase in the history of the study of free-living nematodes was devoted to their collection and morphological observation. For these millimeter-sized animals, this phase started with the advent of microscopy in the 17th century. The inventor of the microscope, Antonie van Leeuwenhoek, himself reported in 1676 observations in old wine of "small living Creatures, shaped like little Eels" (van Leeuwenhoeck, 1676)-perhaps the "vinegar eel" Turbatrix aceti. In this first phase, authors generally aimed to describe and classify species, while also providing some information on their habitats. Most studies included a comparison of many different species, such as the 1866 monograph by Schneider (1866).

A second historical phase began at the end of the 19th century, when nematodes started to be used as model species to study general biological mechanisms. Observations were often supplemented by basic experimentation. As one of the main properties of free-living species was their ability to be cultured continuously, many authors first dealt with culture methods and observations of the lifecycle. A few conducted experiments, which could span one or several generations. This phase was often the work of solitary researchers, some of amazing quality. A key figure of this phase is Emile Maupas, a professional archivist and librarian living in Algiers and working on biology as a personal interest (see Appendix 3, Section 18.1). He was the first to isolate and name Rhabditis elegans (now 
Caenorhabditis elegans) (Maupas, 1900). He described nematode species, not for the sake of systematics, but for their use in more general biological studies. Specifically, his two important articles on free-living nematodes concerned: 1) the molts and the alternative development via the dauer larvae ("enkysted larvae") (Maupas, 1899), and 2) the modes of reproduction and their variations (Maupas, 1900). Maupas had already used C. elegans in the 1899 publication, and more formally described the species in his 1900 article. In the latter study, which particularly focused on hermaphroditism, Maupas studied about 20 species. However, C. elegans was already the first and most developed example and his primary reference species. Compared to previous authors, Maupas can be distinguished by his use of a more experimental approach, albeit rudimentary. He was the first to perform crosses to try to analyze mechanisms of sex determination. After him, Eva Krüger, Paula Hertwig, Karl Belar, and Hikokura Honda each independently studied the reproduction and cytology of different rhabditid species (Krüger, 1913; Hertwig, 1920; Belar, 1923; Belar, 1924; Honda, 1925). In particular, Honda isolated C. elegans again and determined that hermaphrodites have six pairs of chromosomes. At this time, individual biologists worked on a wide variety of organisms. For several, such as Otto Bütschli, Maupas, and Belar, this additional focus included protists, especially ciliates.

A third historical phase in free-living nematode research started in the 1940s, with the birth of the first teams who used free-living nematodes as model organisms, headed by Victor M. Nigon and Ellsworth C. Dougherty (see Appendix 3, Section 18.4 and Section 18.5). Nigon was led to nematodes by the vagaries of circumstances. As a young student during the war, he needed to work at home, far from the university, and his Professor, Albert Vandel, suggested that he work on free-living nematodes. Vandel was aware of the potential utility of these species through the work of Maupas and Belar. Dougherty, who had so far mostly studied parasitic nematodes, joined Nigon in Paris then Lyon to perform experimental work. He focused on C. briggsae after having obtained a culture of this species from Margaret Briggs. The two teams of Nigon (in Lyon) and Dougherty (in California) remained in close contact during this period. Devoted to the analysis of reproduction and nutrition, respectively, they solved several basic problems. First and foremost, culture conditions and experimental methods were refined, increasing the reproducibility of experiments. Also, the laboratories began to devote more effort to focus on a smaller number of model species. Following the example set by Thomas Hunt Morgan in Drosophila, Nigon succeeded in setting up strictly controlled culture conditions using regular transfers of single animals that served as the basis for more rigorous studies. Most importantly, crosses of selfing species such as C. elegans were mastered (Nigon, 1943) and the chromosomal basis of $C$. elegans sex determination was determined using cytological studies of meiosis and making use of a tetraploid line (Nigon, 1949a; Nigon 1951a). The team leaders were conscious of the exceptional potential of free-living nematodes. Despite this obvious promise, their projects did not then succeed in convincing funding authorities and assembling specialists in complementary fields. This meant that by 1952 Nigon started to develop other scientific projects, apparently more in step with the development of molecular biology, while his student Jean-Louis Brun continued on C. elegans, on a difficult project of laboratory adaptation. Dougherty's problems in getting financial support continued, which led him to criticize funding practices in the United States (see Dougherty, 1959, p. 45).

The fourth phase in the development of free-living nematodes as model organisms started for C. elegans in 1974, with the first publications by Sydney Brenner and his collaborators. This phase is perhaps the best known. Sydney Brenner, one of the founders of molecular biology in the 1950-60s and a very experienced and well-known bacterial geneticist, decided to start a new research program in developmental biology and neurobiology (Brenner, 1988). Conscious of the limits of bacteria and of the need to bring the genetics of animals towards molecular biology, he was tempted by free-living nematodes, and was particularly inspired by Richard Goldschmidt's work (Goldschmidt, 1908; Goldschmidt, 1909) on neuronal cell invariance and nervous system connectivity in Ascaris, as well as perhaps being influenced by Dougherty whom he met in Berkeley (Brenner, 2001). Brenner experimented with many nematode species, and isolated a number of new strains, before finally settling on C. elegans around 1966 (Brenner, 1966/1981). The fame enjoyed by Brenner and the place where he worked (the Medical Research Council (MRC) Laboratory of Molecular Biology in Cambridge, UK) allowed him to succeed in obtaining the funding that his predecessors had sought in vain and he could recruit an outstanding set of colleagues to work on this new project. Brenner's knowledge of bacterial genetics and molecular biology enabled him to overcome some of the obstacles that had been challenging to Nigon and Dougherty. He thus opened up a field full of new perspectives and innovation.

The study of free-living nematodes has been built upon work conducted during each of these four phases of research. The present article will focus primarily on work with free-living nematodes during the second and third phases of their development as model organisms. We chose to emphasize experiments using C. elegans (and $C$. briggsae), with particular attention on studies of variation in modes of reproduction. Keeping track of work using a variety of free-living nematode species also helps to provide context for the progressive focus of scientists on $C$. 
elegans. A more extensive review covering the work of additional authors, especially in developmental biology, can be found in Nigon (1965), in French. The individual sections below, rather than following a historical thread across these two phases, are divided along different biological questions. Work conducted during the fourth phase has solved some of the outstanding questions from the earlier era and will be referred to as a complement at the end of each section or subsection ("In today's light"). We will end with an epilogue, which will reflect back on some of the early developments that remain unexplored today (2015) and may still open new horizons.

Regarding taxonomy, note that nematode genus and species names have varied during this historical period. For the ease of reading, we generally use the current taxonomy. A table containing old and present names is found in Appendix 1 (Section 16). For example, Caenorhabditis elegans was called Rhabditis elegans, when it was formally described by Maupas (Maupas, 1899; Maupas, 1900). The Rhabditis genus was then subdivided into subgenera by Günther Osche (1952), and these subgenera raised to the status of genera by Dougherty (1953). Many species that belonged to the Rhabditis genus before 1950 are thus now in different genera, called Caenorhabditis, Oscheius, Pellioditis, Mesorhabditis, etc. Similarly, the genus Pristionchus was previously part of Diplogaster (Sudhaus and Fürst von Lieven, 2003). See Kiontke et al. (2007) for a current phylogeny of the rhabditids, and Sudhaus (2011) for the latest systematic denominations. Phylogenetic analysis shows how parasitic and free-living nematodes are evolutionarily intermingled (Blaxter et al., 1998). Only a few strains isolated and studied before the 1970s-such as C. elegans Bergerac-were maintained in culture past this date and later frozen down. Current collections however contain new isolates of most previously studied species.

\section{Methods}

\subsection{Culture}

Until 1920, most authors employed culture methods based on those of Maupas (1900). The container was a hollow slide, a watch glass or a Petri dish, maintained in a humid chamber or under glass sealed with paraffin oil. The medium was a layer of water, thin enough to allow for oxygenation. Food was provided by pieces of decomposing meat, the nematodes feeding on the bacteria that grew on them. Some authors tried peptone solutions with variable success. Haven Metcalf was the first to employ agar media (Metcalf, 1903).

When comparing the culture methods used for free-living nematodes with those in practice in Drosophila genetics, Nigon noticed major differences (Nigon, 1943). In Drosophila crosses, progenitors were isolated by pairs and transferred every day to new containers. They thus received constant and reproducible conditions. For nematodes, transfer of isolated progenitors could be achieved more readily by replacing the liquid medium with an agar medium. Nigon thus poured an agar medium in large drops on preparation slides that were maintained in humid chambers (Figure 1). The medium was seeded with micro-organisms that served as food: first yeasts, easily obtainable anywhere, which were later replaced by defined bacterial cultures by Dougherty and Brun. The animals developed in a confined medium because those venturing outside the drop onto the glass surface were threatened by rapid desiccation. Every day the adult(s) were moved to a new drop, leaving the progeny produced during 24 hours on the previous drop. In some difficult research settings (Bergerac), the animals were followed with the naked eye, transported with a wood needle, with a possible a posteriori confirmation of their number and sex using a low-magnification compound microscope (Nigon, 1943) (Figure 1). In more comfortable laboratory conditions, all manipulations were carried out under the dissecting microscope (Nigon, 1949a). 


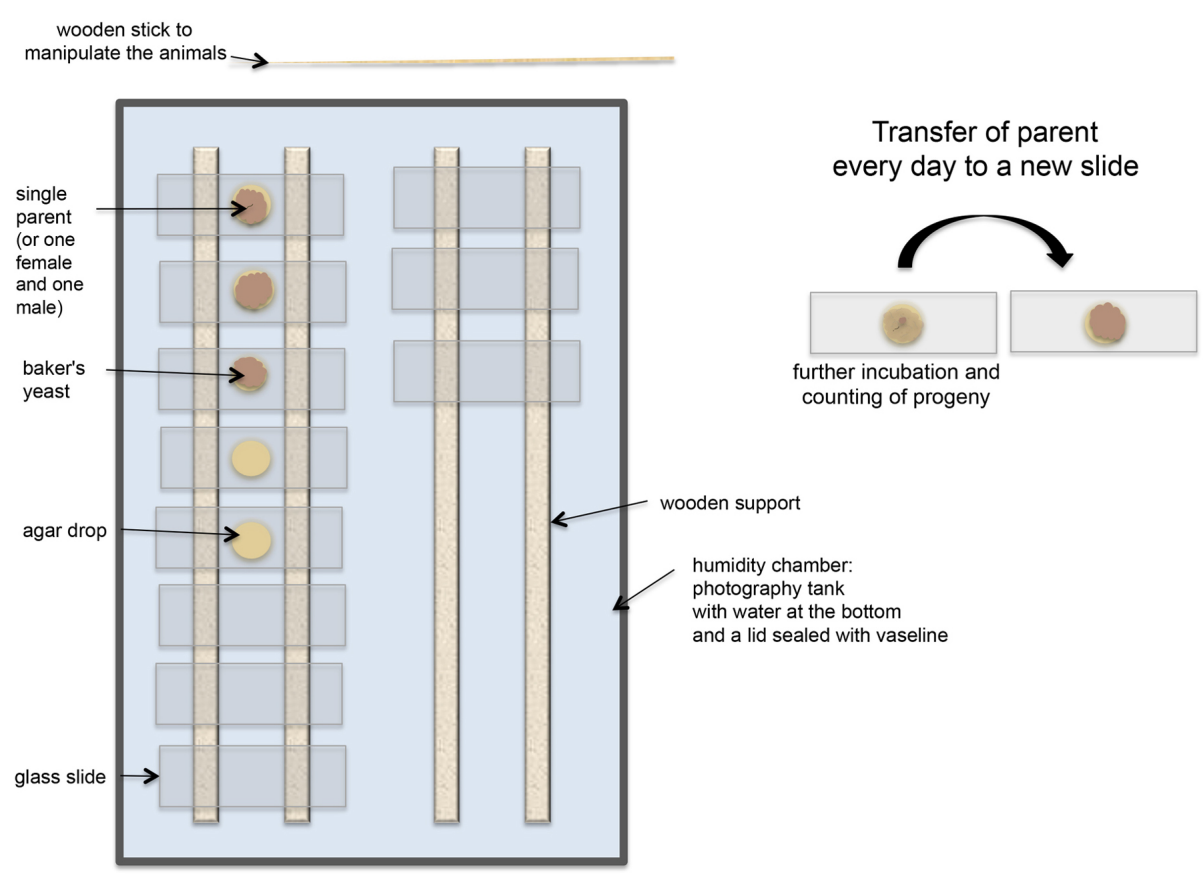

Figure 1. Organization of the nematode culture by Nigon $(1943,1949)$. The parents were transferred daily to a new slide. The drawing corresponds to the setup in Bergerac. Later in the laboratory, cultures on slides were placed vertically in tanks for histological slides.

Following procedures established for C. briggsae by Margaret Briggs in her 1946 Master thesis at Stanford University, Dougherty and Calhoun (1948b) also used an agar medium, seeded with a culture of E. coli. By treating isolated animals with antiseptic solutions, they achieved monoxenic cultures.

Dougherty and his collaborators devoted the greatest part of their activity to seeking a chemically defined culture medium. Their plan was to look for nutritional mutants, following the work of George Beadle and Edward Tatum with Neurospora (Dougherty and Calhoun, 1948b). A first step towards this goal was to succeed in employing an axenic liquid medium containing either a chick embryo or a liver extract (Dougherty, 1950; Dougherty et al., 1959), but the composition of these media still remained largely undetermined.

The next objective was thus to create a fully chemically defined medium. In their last publications on the subject (Dougherty et al., 1959; Nicholas et al., 1959), the authors described a fully defined medium (GS-25), which could sustain the development of larvae into adults, with, only once, the production of an F1 generation. The addition to this medium of $1 \%$ of a protein fraction of liver (LPF-C) allowed them to obtain up to eight successive generations. They concluded with the following sentence: "The axenic cultivation of $C$. elegans on a chemically defined medium still presents challenges. Undoubtedly, more workers are needed.” (Dougherty et al., 1959).

\section{In today's light}

Nigon succeeded in setting up strictly controlled culture conditions, using an agar medium and regular transfers of single animals, where the free-living nematodes could grow and mate (Nigon, 1943). Culture conditions and nematode transfer methods have not greatly changed since, but have tended to converge to one standard agar medium (called Nematode Growth Medium or NGM agar, Maintenance of C. elegans), now poured into plastic Petri dishes. Following Brenner (1974), C. elegans is fed monoxenically a growth-restricted Escherichia coli strain called OP50, which allows for better observation of the animals on the E. coli lawn (Maintenance of C. elegans). Strains can now be kept frozen, a major improvement in terms of strain maintenance and genotype stability.

The serious efforts called for by Dougherty et al. (1959) have been made: the nutritional requirements of $C$. elegans and C. briggsae are now understood and a chemically defined axenic culture medium has been devised. An excellent analysis of subsequent studies performed by Dougherty's and other teams is available in Nicholas (1975), including those by W.F. Hieb who had worked in Dougherty's laboratory (Hieb and Dougherty, 1966). Specifically, C. elegans was found to require some external sterol source (Hieb and Rothstein, 1968; now added as cholesterol in the agar medium, because E. coli does not provide it), as well as heme (Hieb et al., 1970; Rao et al., 2005). In 
modern standard culture conditions, the heme is provided by E. coli, but in axenic conditions, heme must be provided, together with a carrier protein (Buecher et al., 1970; Vanfleteren, 1974). A chemically defined medium could finally be devised, where the worms could proliferate, albeit more slowly (Lu and Goetsch, 1993; Szewczyk et al., 2003). This medium is rarely used, although it is famous for space shipping and C. elegans survival after the Columbia space shuttle disaster (Szewczyk et al., 2005). Nutritional requirements of C. elegans have been studied by Nancy Lu and her team in Berkeley and San Jose, California (Lu et al., 1977; Lu et al., 1983; Lu and Goetsch, 1993; Perelman and Lu, 2000; Balachandar and Lu, 2005; Zhao and Lu, 2008; Xiong and Lu, 2011). The genetic screens for nutritional mutants that Dougherty dreamt of are still to come, but other physiological studies are promising.

\subsection{Cytological methods}

Earlier authors, such as Maupas, were able to observe live animals in the microscope. To visualize chromosomes, Eva Krüger was the first in 1913 (after Boveri on Ascaris) to embed animals in paraffin, section and stain them with hematoxylin. This technique did not allow an easy analysis of the succession of cellular structures during gametogenesis of a given individual. Nigon (Nigon, 1946; Nigon, 1949a) then established a method that overcame this difficulty. A microknife was built by breaking the edge of a razor blade with forceps. This small blade was inserted into the chuck of a watchmaker tool. An individual, male or female, was transported onto a dry preparation slide into a small waterdrop. A strike of the microknife skillfully aimed at the nematode's flanks made it expel its internal organs. A drop of Carnoy fixative was immediately added, ensuring at the same time the fixation of the animal and the coagulation of the expelled substances. After this surgery, the dissected animal was stuck on the glass surface, with its internal organs spread out. The preparation was subjected to the Feulgen reaction, which colored DNA bright red. After a brief drying step, a drop of mounting medium and a coverslip were added, with no need for sectioning. Except for the Feulgen reaction, which dates from the 1930s, this method was derived from a technique that had been in common use in protistology for a long time. It is thus somewhat surprising that protistologists such as Maupas and Belar did not apply it to nematodes. Using this method, one can follow the successive steps of gametogenesis as they occur along the genital tract of a single individual (Nigon and Brun, 1955) (see Section 4.3). A few such preparations were sufficient to capture all steps of gametogenesis and fertilization. Phase contrast microscopy further helped visualization. As was already achieved by previous authors, one could follow and film the developmental process in different focal planes, on isolated eggs or on the live animal without prior dissection (Nigon et al., 1960).

Later, the same razor blade technique was applied to animals after incubation with radioactive (tritiated) thymidine for a few hours. The animals were dissected and covered with a sensitive film in the dark room. DNA synthesis could thus be followed during gametogenesis (Nonnenmacher-Godet and Dougherty, 1964).

\section{In today's light}

The razor blade technique described by Nigon is still being used for gonadal and intestinal staining, as well as for egg and early embryo isolation. Advances in microscopy have been crucial. Nomarski microscopy allowed the nucleus of each cell to be followed in a live animal and rendered possible the determination of the entire cell lineage by John Sulston et al., from egg to adult (Sulston and Horvitz, 1977; Sulston et al., 1983). 4D-microscopy movies (with different focal planes over time as in Nigon et al., 1960) are now achieved through automatic recording, which further facilitates the determination of cell lineages. The development of electron microscopy was also crucial, especially to determine the wiring of neurons (White et al., 1986). Fluorescent Hoechst staining or labeling of histones with Green Fluorescent Protein are now used to observe DNA in live animals. Specific staining of a given sequence of nucleic acids or of a protein is now possible by in situ hybridization (Single molecule fluorescent in situ hybridization (smFISH) of C. elegans worms and embryos), immunostaining (Immunohistochemistry), and/or transgenic fusions of endogenous proteins with fluorescent proteins (Reporter gene fusions).

\section{Hermaphroditism: selfing and crossing with males}

Since the late $19^{\text {th }}$ century, nematodes have been model organisms, not only to study meiosis and fertilization, but also variation in reproductive modes. Maupas' 1900 major opus (over 160 pages, in French), entitled "Modes and forms of reproduction of nematodes", focused on several types of variation relative to the standard male-female reproduction. The main emphasis was on hermaphroditic reproduction, and particularly that of $C$. elegans. Interest in the diversity of nematode reproductive modes and the underlying cellular and chromosomal processes run through the first half of the 20th century-successively through Eva Krüger, Paula Hertwig, Karl Belar, Frank Armitage Potts, and Hikokura Honda to Victor M. Nigon. A second landmark article was Nigon's 1949 publication, entitled (in 
French) "Modes of reproduction and sex determination in some free-living nematodes" (Nigon, 1949a). This 135-page article demonstrated the XX/X0 chromosomal basis of sex determination in C. elegans, and observed $\mathrm{X}$-chromosome non-disjunction during meiosis in Oscheius dolichura hermaphrodites. Less known today are his analyses of meiosis and fertilization in other free-living nematodes, including pseudogamy in Mesorhabditis belari. We review hermaphroditism in this section, chromosomal sex detemination in Section 4, and parthenogenesis (including pseudogamy) in Section 5.

\subsection{Protandric hermaphroditism as a modification of the female}

Many free-living nematodes have separate sexes (gonochorism). The gonad of females produces oocytes, that of males spermatozoa. After mating, spermatozoa are stored in the spermatheca of the female gonad, usually located between the site of oocyte maturation and the uterus. When oocytes mature, they pass one after the other through the spermatheca where they are fertilized.

A key observation of Maupas (1900) was that in nematodes, the hermaphrodite individuals display a female body morphology. In their germ line, gametogenesis begins in early adulthood with the production of sperm, which is then stored in the spermatheca (Figure 2). Oogenesis then occurs in a chain of successive maturations along the gonadal axis, as in true females. This mode of reproduction is thus called protandric hermaphroditism. As in true females, the most mature oocyte passes through the spermatheca and becomes fertilized by self-sperm. In sum, the hermaphrodites are similar to the females as far as the soma is concerned and only differ in germ line behavior. One must thus distinguish in these species between sexual differentiation of the soma and that of the germ line (Maupas, 1900; Nigon, 1949a).
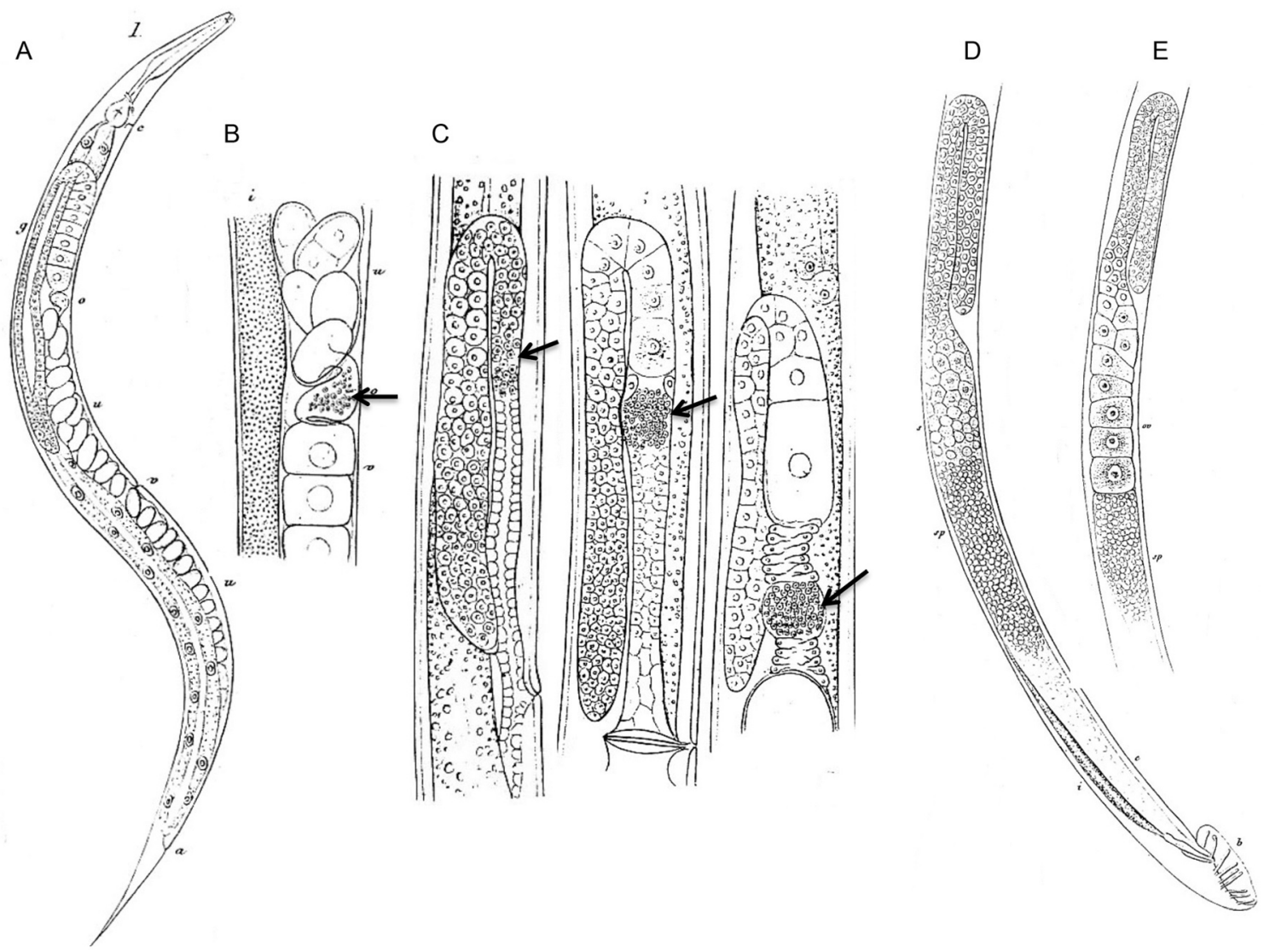

Figure 2. The first drawings of adult C. elegans hermaphrodites and males, their reproductive organs and gametes. Modified from Maupas (1900). (A) $C$. elegans hermaphrodite. $v$ : vulva; $o$ : oviduct $=$ spermatheca; $g$ : ovary; $c$ : nerve ring and excretory pore; $a$ : anus. (B) Detail of the $C$. elegans hermaphrodite gonad where oocytes are fertilized by sperm stored in the oviduct (arrow) and then pass into the uterus. $o$ : oviduct; $v$ : vitellogen; $u$ : uterus; $i$ : intestine. (C) Successive stages of spermatogenesis (arrows) in the young adult hermaphrodite, from left to right; shown here in $O$. dolichura, another hermaphroditic free-living nematode. (D) Reproductive system of the $C$. elegans male. $s$ : spermatids; $s p$ : spermatozoa; $b$ : bursa and spicules; $c$ : deferent canal; $i$ : intestine. (E) Detail of an 'aberrant' $C$. elegans male with a hermaphroditic germ line: large oocytes ('oo') can be seen in addition to small sperm cells below ('sp'). The different panels use various magnifications that are not indicated here. 
In most hermaphroditic species, male individuals are also produced at a frequency of around 0.1-1\%. These males produce sperm of identical shape as those produced by hermaphrodites. According to Maupas, however, these males (here of $C$. elegans) appear to have little sexual instinct: "I actually doubt that any male ever occurs in nature. With animals so little lustful as the males of our Rhabditis, the very special conditions of sequestration in which I maintained them must be required to induce some of them to mate.". They often show variability at the level of the male tail and spicules. Some intersex males that form oocytes in their testis have been observed (Maupas, 1900; Nigon, 1949a) (Figure 2E).

Maupas tested the ability of $C$. elegans to reproduce for many generations through self-fertilization without outcrossing. He cited Charles Darwin on the fact that outcrossing seemed favored in nature: "Nature thus tells us, in the most emphatic manner, that she abhors perpetual self-fertilisation" and "No hermaphrodite fertilises itself for a perpetuity of generations". Testing Darwin's suppositions, Maupas transferred 20 virgin C. elegans hermaphrodites per generation, during 50 generations. He observed no degeneration. (His experiment was interrupted by summer, the high temperature likely causing sterility of the line.) He concluded that outcrossing is not necessary, at least in the short term.

\subsection{Variation in hermaphroditism among different species}

Maupas' article compared reproduction of several hermaphroditic nematode species (Maupas, 1900). Different species displayed at the level of the sexual structures and of the mode of reproduction a considerable variability of modalities, sometimes within the same species:

1) Maupas found Reiterina viguieri to be the closest to the reproduction of gonochoristic species, with about $5 \%$ males. These males were active and often found mating. Among females (the term included all somatically female individuals, including hermaphrodites), about one in ten was without sperm. This species thus included three sexual forms: hermaphrodite (most common), female and male. Maupas did not systematically study the progeny of different types of females, crossed or not with males.

2) In Rhabditis marionis, Maupas observed a lower frequency of males, $0.76 \%$. Many hermaphrodites of this species laid fertilized and unfertilized eggs at the same time. Maupas hypothesized that the presence of unfertilized eggs (also observed in Rhabditis duthiersi) may be due to the fact that spermatogenesis only occurred in one of the two ovaries. This remains unclear as Maupas did not directly assess the absence of sperm with his microscope and this has not been studied since.

3) In Rhabditis guignardi, Maupas observed the smallest proportion of males (0.015\%). Hermaphrodites produced many sperm and on average 520 progeny per individual-thus, much more than other hermaphroditic species (usually 300 or less).

After Maupas, F. A. Potts (Potts, 1910), based in England, studied new hermaphroditic species, which he described succinctly (Pristionchus linstowi, Pristionchus maupasi, and Rhabditis gurneyi). By culturing P. maupasi unmated hermaphrodites on different peptone solutions in different instances and locations, Potts noticed in their progeny large variations in male frequencies, ranging between a very low value and $30 \%$. He did not control several factors, such as temperature, which may have caused variations in observed male frequencies. Potts reported that $R$. gurneyi females first produced unfertilized oocytes, then fertilized ones. He interpreted these results to mean that some individuals displayed several successive phases of spermatogenesis. Like Maupas' hypothesis above, Potts' hypothesis on $R$. gurneyi was never verified by a direct observation of spermatogenesis, and the laying of unfertilized eggs may be explained by factors other than the lack of sperm. His results, thus, remain unclear and have not been reproduced.

\subsection{Crosses in hermaphroditic species}

At the time of Maupas' work, a chromosomal basis for sex determination was not known. Maupas (1900) attempted matings between the hermaphrodites and the rare males that they produced via self-fertilization. To avoid interference by hermaphrodite sperm, Maupas used old females, presumed to have exhausted their own sperm, as inferred by the fact that they had ceased to lay developing embryos for at least 24 hours. Maupas mostly used $C$. elegans for his experiments. He set up eight cultures by mixing several hermaphrodites and several males. Two did not yield any progeny, while the six others produced 127 males and 147 hermaphrodites, thus approximately an equal number. Some resulting males were intersex, with oocytes in the testis (as shown in Figure 2E). When 
isolated, the F1 hermaphrodites gave rise mostly to hermaphrodites, with only $0.23 \%$ males. The F1 males were associated with other old hermaphrodites in five cultures (each with 4-11 males). In this second generation, no progeny were obtained. Seven other hermaphroditic species tested by Maupas in the same manner were infertile in the first generation of mating pairs. In $R$. marionis and $R$. duthiersi, the old hermaphrodites placed with males yielded only further hermaphrodites. In summary, Maupas succeeded in mating C. elegans for one generation, which indicated that outcrossing with males may increase male production. But his results could not be repeated at the next generation. He concluded that the F1 males were degenerate and sterile. However, as explained below, his culture conditions may not have been optimal for mating.

Hikokuro Honda tried to reproduce Maupas' experiments (Honda, 1925). He isolated C. elegans (likely this time in North America where he worked). He noticed that his C. elegans strain, even after having stopped producing fertile eggs for 24 hours, may still sometimes produce progeny-thus possibly explaining why Maupas' mating experiments with old $R$. marionis and $R$. duthiersi hermaphrodites did produce progeny but only hermaphrodites. In C. elegans, Honda did not obtain any males in his mating attempts. However, with $O$. dolichura he obtained males in the first generation. He correctly observed six bivalents in the oocytes and spermatids of C. elegans.

Nigon (Nigon, 943; Nigon, 1946; Nigon, 1949a) was the one who succeeded in carrying out crosses reproducibly in species with hermaphrodites and rare males-C. elegans and $O$. dolichura. Nigon isolated C. elegans from garden soil in Bergerac, France (see Appendix 2, Section 17 and Appendix 3, Section 18.4). In contrast to his predecessors, he maintained his cultures by isolating animals individually at each generation, on a drop of agar on a slide (Section 2.1, Figure 1). When obtaining a rare male from an isolated hermaphrodite, he placed it with one of its sisters. In some cases, the offspring was exclusively hermaphroditic-the males may have been sterile. In other cases, the progeny contained approximately the same number of males and hermaphrodites. Most males of the second generation were fertile (93\%) and again gave rise to both hermaphroditic and male progeny. From the third generation, almost all males were fertile. Males could thus be propagated through crosses at will.

In summary, Maupas used group cultures of several (5-20) males with as many aged hermaphrodites. His spontaneous males obtained from hermaphrodite self-fertilization were often fertile, while all males of the second generation seemed to be sterile. By contrast, in Nigon's experiments, young males were associated with young hermaphrodites. The spontaneous males were often sterile, but from the third generation, almost all were fertile.

\section{In today's light}

Why were the mating experiments successful in the hands of Nigon and not of his predecessors? Several factors may have contributed. First and perhaps most importantly, the culture and mating conditions that Nigon set up were highly controlled and allowed reproducible experiments, including crosses with single hermaphrodites and males, on an agar medium incubated in a humid chamber (Section 2.1). In contrast, Maupas was culturing large populations in depression slides, in water to which rotten meat was added. Honda also used a liquid medium, in which C. elegans males could perhaps not mate. Second, Maupas used aged hermaphrodites, which may have been too old to reproduce. The first point is likely the main factor. The control of mating of males with hermaphodites is the basis for modern C. elegans genetics (Dougherty, 1963; Brenner, 1974).

The odd three-sex situation described by Maupas in Reiterina viguieri has been found again in "Rhabditis"sp. SB347, related to Reiterina viguieri (see Section 8.2).

\subsection{Biological species test through crosses: C. briggsae discovery}

The use of crosses between males and hermaphrodites allowed the testing of cross-fertility between isolates from nature. The lack of males in the progeny of a cross between males of strain A and hermaphrodites of strain B (or reciprocally) indicated that the two strains were not cross-fertile. An absence of cross-fertility indicated the presence of two different species, provided within-strain controls were positive. Maupas had attempted such hybridization tests between morphologically diverse species, without success. Some matings between morphologically diverse species occurred with the deposition of a copulatory plug, while a few yielded arrested embryos or gonadless adults (Maupas, 1919). Conversely, using a strain that was morphologically difficult to distinguish from the Bergerac isolate, Dougherty and Nigon discovered through crosses a new species, which they named $C$. briggsae.

The new strain had been collected in 1944 by Margaret Briggs, then a student at Stanford University, using a peanut butter enrichment procedure to initiate the culture (Dougherty and Nigon, 1949; Nigon and Dougherty, 1949; 
Briggs Gochnauer and McCoy, 1954; procedure described in Dougherty and Calhoun, 1948b). This culture was passed to E. Dougherty, who first called it "R. elegans" because of its close morphological similarity (Dougherty and Calhoun, 1948b). Briggs continued to work on C. briggsae culture conditions, including its response to antibiotics (Briggs Gochnauer and McCoy, 1954). This strain is still available as DR1690 (Fodor et al., 1983; McGrath et al., 2011).

By crossing the Berkeley strain to the $C$. elegans Bergerac strain, Nigon and Dougherty found no males in the progeny. In addition, using a micro (dumpy) mutant (see Section 9.2) in the heterozygous state in males, they did not see the mutation segregate in the grandprogeny of hermaphrodites (Dougherty and Nigon, 1949; Nigon and Dougherty, 1949). Some phenotypic features of the two cultures were noticed: the Berkeley strain displayed a lighter color of intestinal granules, a smaller number of embryos in the uterus, fusion of sensory male rays 3-4, and a slower growth at $12{ }^{\circ} \mathrm{C}$. Comparing morphological measurements with those of Maupas for C. elegans, they concluded that the California strain was the new species and they named it Rhabditis briggsae (Dougherty and Nigon, 1949), now Caenorhabditis briggsae. They concluded that there may be many cryptic species that cannot be distinguished morphologically in the genus Rhabditis.

The same procedure was used by Nigon to assign a new Caenorhabditis isolate from Bristol to C. elegans. The Bristol strain was isolated in 1951 from mushroom compost by Warwick Nicholas during a nematology course organized by L. N. Staniland in Bristol (Nicholas, 1975; Hodgkin and Doniach, 1997; McGrath et al., 2011). This strain passed through the hands of nematologists such as Osche (Nicholas et al., 1959) and B. G. Chitwood (Hansen et al., 1960), and was definitively identified as C. elegans by Nigon, through positive hybridization to the Bergerac strain (Nicholas et al., 1959).

\section{In today's light}

As many more morphologically similar species of Caenorhabditis have been found, the biological species concept for species definition has been further implemented in the genus by Sudhaus and Kiontke (2007) and Félix et al. (2014). A gonochoristic Caenorhabditis species that is morphologically similar to C. elegans and C. briggsae was described in 1974 as C. remanei (Sudhaus, 1974). Dozens of new gonochoristic species have been found since, as well as a third hermaphroditic species, C. tropicalis (Kiontke et al., 2011; Félix et al., 2014). The finding of closely related species with partial hybridization properties has already allowed studies of genetic incompatibility and speciation in Caenorhabditis to start (Woodruff et al., 2010).

\subsection{Evolution of selfing from male-female ancestors}

Hermaphroditism in nematodes appeared to be independently derived several times from the gonochoristic mode via evolutionary modification of the female germline (Maupas, 1900). This suggested that the evolutionary transition from one mode to another was easy.

This idea was reinforced in later experiments by Hedwig Hirschmann on the gonochoristic Pristionchus lheritieri (then Diplogaster lheritieri) (Hirschmann, 1951). Hirschmann established parallel lines of $P$. lheritieri through inbreeding (brother $\mathrm{x}$ sister) of a strain she had isolated from nature; these lines rapidly decreased in fertility, a case of inbreeding depression. In the F4-F5 generations, she observed some self-fertile animals as well as other types of intersex individuals. With the self-fertile animals, she started a line that reproduced through self-fertile, protandrous hermaphrodites and rare males. Four replicates of inbreeding confirmed this transition in reproductive mode.

She described these selfing strains under a new species name, "Diplogaster biformis". She also repeatedly isolated from nature selfing strains with a similar morphology. She tested a selfing strain for reproductive isolation with $P$. lheritieri, and found that they did not produce cross-progeny. But it is unclear whether she used the selfing laboratory descendants of $P$. lheritieri or the morphologically similar selfing species that she had found in nature (in fact likely P. maupasi, Osche, 1954; Triantaphyllou and Hirschmann, 1964). Thus, it is unclear whether the selfing species obtained by inbreeding was reproductively isolated from its gonochoristic parent.

Yet, her inbreeding experiment suggests that the evolutionary transition from obligate outcrossing to selfing may be obtained in the laboratory, at least in some cases, when starting with natural populations of gonochoristic species. 
In today's light

These findings have not been reproduced so far, but we now know that in the gonochoristic C. remanei, hermaphroditism can be obtained through targeted mutational change in just two independent pathways (Baldi et al., 2009).

\section{Sex determination and meiosis in sexual species}

\subsection{Chromosomal sex determination in gonochoristic species}

Cultures of gonochoristic free-living nematodes generally contain the two sexes in approximately equal proportion based on an XX/XY or XX/X0 chromosomal sex determination. The cytological study of chromosomes of free-living species by Nigon showed either no difference in chromosome number between males and females (Panagrolaimus rigidus, Nigon, 1949a), suggesting a XX/XY mechanism, or one less chromosome in males (Pellioditis pellio, Hertwig, 1920; Pelodera strongyloides, Nigon, 1949a). During P. strongyloides spermatogenesis, all primary spermatocytes were observed to bear a single X and "reduction" of the X chromosome occurred at the second meiotic division (Nigon, 1949a). XX/X0 sex determination had been found previously in some parasitic nematode species, such as Angiostomum nigrovenosa (now called Rhabdias bufonis; Boveri, 1911; Schleip, 1911). Note that some male-female plant parasitic nematodes display environmental sex determination (Triantaphyllou and Hirschmann, 1964).

\subsection{Chromosomal sex determination in hermaphroditic species}

In hermaphroditic species as in gonochoristic species, mating of males with hermaphrodites gave rise to males and hermaphrodites in similar proportions (Nigon, 1949a). Cytology, which required destroying the animals, was best performed on young males before they mated. Males could, however, be examined in the following generations and were shown to carry one less chromosome than hermaphrodites (Figure 3). These results indicated that sex determination in hermaphroditic species had a chromosomal basis, with an XX/X0 system, as in the related gonochoristic species. This XX/X0 system was observed for both O. dolichura (Nigon, 1946) and C. elegans (Nigon, 1949a).
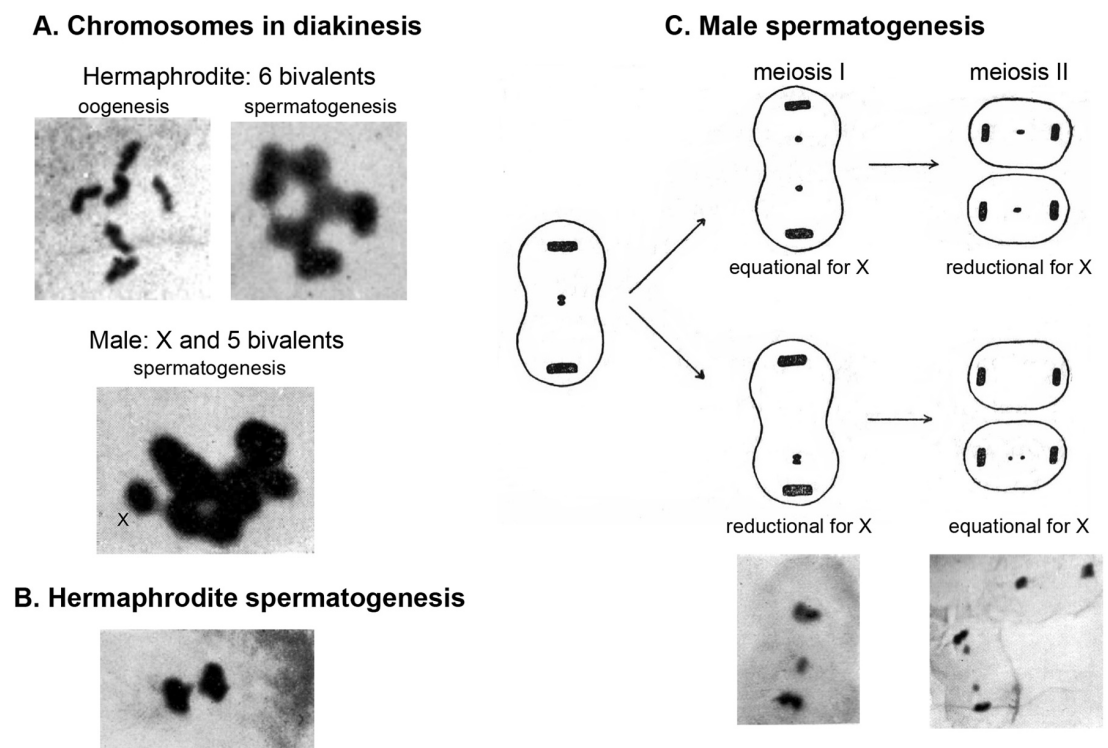

Figure 3. The sexual chromosome in C. elegans: cytological analysis. Assembled from Nigon (1949). Feulgen staining of chromosomes. (A) The chromosomal content was observed in prophase of meiosis I to be of six bivalents in hermaphrodites, and of five bivalents and one univalent (the $\mathrm{X}$ chromosome) in males. The chromosomal content was more difficult to see on pictures of spermatogenesis. (B) Delay of the X chromosome (small dot) in the first division of hermaphrodite spermatogenesis. This delay was seen more clearly in O. dolichura (not shown here). (C) Two possible behaviors of the $\mathrm{X}$ chromosome in male meiosis, with reduction at the first or second division. Note also the delay in $\mathrm{X}$ chromosome separation in anaphase. The pictures below were taken in $C$. elegans. In $O$. dolichura, the top case where the first meiotic division is equational for the $\mathrm{X}$ chromosome (reductional for the autosomes) was most frequent. 
What about the chromosomal content of the rare males coming from self-fertilization of hermaphrodites? Nigon (1949a) found it impossible to both examine chromosome number in a male and test its fertility. Fertile males were likely X0 because when crossed to hermaphrodites they gave rise to progeny of which half carried 11 chromosomes (X0) and the other half 12 chromosomes (XX). What about the sterile males that were produced by selfing hermaphrodites, and to some extent by hermaphrodites, crossed to a fertile male? Several experiments attempted to answer this question, including trying to visualize spermatogenesis in the hermaphrodites and to obtain more males in the progeny of selfed females. Both are detailed below.

A special behavior of one chromosome pair, likely the sex chromosomes, was observed during hermaphrodite spermatogenesis in O. dolichura (Nigon, 1946; Nigon, 1949a). In some spermatocytes, a standard symmetrical separation of all bivalents (homologous chromosomes) took place at anaphase, while in some others, separation of one bivalent was delayed. This delay could possibly result in this bivalent entirely segregating into one daughter cell, or being lost in the residual body discarded during sperm maturation. Assuming that the delayed bivalent corresponded to the $\mathrm{X}$ chromosome, one could thus obtain nullo-X spermatozoa. Their fusion to a normal oocyte would give an egg with a single $\mathrm{X}$, producing a male. To be sure of the identity of this delayed chromosome, a marker on this chromosome would have been useful. A similar delay of one chromosome was also observed in $C$. elegans at anaphase of meiosis I, but to a lesser extent (Figure 3C).

Several methods were employed to induce a hermaphrodite to produce a higher incidence of males: submitting the hermaphrodites to a heat shock (for example $25^{\circ} \mathrm{C}$ for several hours) or treating them with a colchicine solution (Nigon, 1949b). When these treatments were applied during the spermatogenesis of hermaphrodites, they led to various chromosomal aberrations that could be observed cytologically. The animals thus treated were often sterile, or had diminished fertility. The progeny included many irregular cases (Nigon, 1949a), including males, some of which were intersexual, a form already described by Maupas (1900), or true female individuals with no trace of spermatogenesis. It may be hypothesized that these abnormalities resulted from abnormal chromosomal contents. The absence of morphological differences or of gene markers among chromosomes was here again the obstacle to further studies.

\subsection{Meiosis in a sexual species, C. elegans}

Nigon and Brun studied chromosomal behavior in meiosis, using Feulgen staining (Section 2.2) and live material. Early meiotic stages could be conveniently studied in adult females and hermaphrodites, as successive stages are aligned along the ovary in a single animal (Nigon, 1949a; Nigon and Brun, 1955) (Figure 4). Nigon and Brun observed that at the tip of the gonad, oogonia underwent mitotic divisions. The oocyte nuclei then entered meiotic prophase I and transited from synapsis to diakinesis along the arm of the gonad (Nigon and Brun, 1955). Fertilization then triggered the two meiotic divisions and polar body formation. 


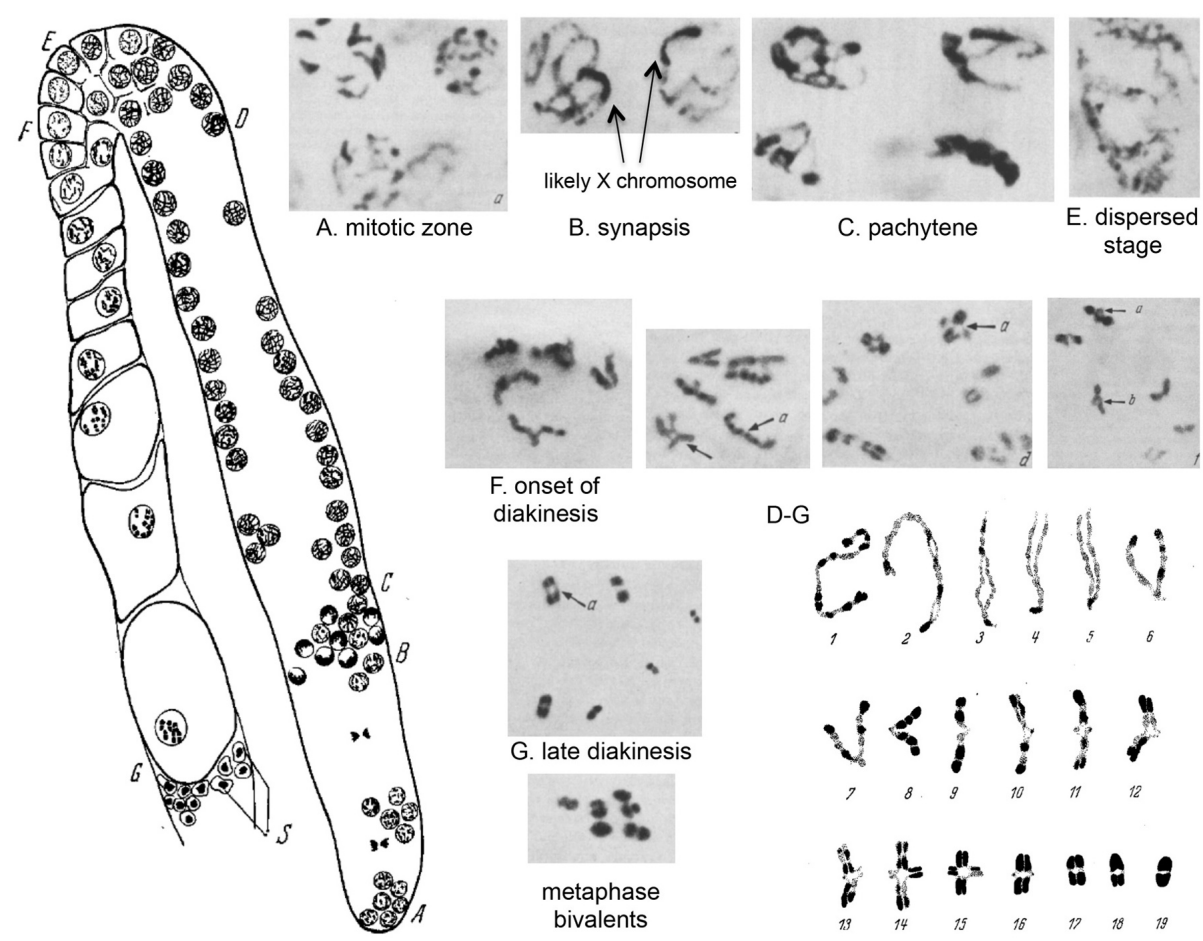

Figure 4. Oogenesis in C. elegans. Rearranged from Nigon and Brun (1955), with permission from Springer Publishing Company. Left: Drawing of an ovary arm in the adult hermaphrodite, showing the successive stages of oogenesis. (A-B) Mitotic zone. (B-C) Synapsis zone. (C-D) Pachytene zone. (D-G) From pachytene to diakinesis. (E) Dispersed (diffuse) stage. (F) Formation of V-like figures. (G) Diakinesis. S: Sperm in spermatheca. Right: Feulgen staining of chromosomes at successive stages, from left to right and top to bottom, some being labeled with a letter corresponding to the gonad zone in the left drawing. The last panel shows 18 putative successive stages for one chromosome, from pachytene to terminal diakinesis. 6-9: V shapes. 13-15: Cruciform tetrads; a shorter arm gives a Phi shape. 18-19: Bivalents.

Chromosome behavior during pairing and recombination was studied during oogenesis in C. elegans (Figure 4) (Nigon and Brun, 1955). Chromosomes were observed to synapse (pair) at the onset of meiotic prophase. One of them often appeared hypercondensed (the X chromosome). After pachytene, the chromosomes entered a dispersed stage (now called the diffuse stage), most easily seen in older adults. During diakinesis, the recombined homologs started separating, usually adopting a V-shape. The chromatids then partially separated (tetrads). Further condensation resulted in two bivalents, which then migrated to opposite poles of the first meiotic division. This scheme was somewhat different from that proposed for species with a distinct rather than diffuse centromere (Darlington, 1937).

The study of spermatogenesis in X0 males was performed by Nigon (1949a), mostly using O. dolichura. As in C. elegans, the diploid number of chromosomes was 12 in hermaphrodites, 11 in males. In the O. dolichura male, the first meiotic division was observed to be most often reductional for autosomes, but equational for the (haploid) $\mathrm{X}$ chromosome, with each chromatid migrating to a different daughter cell at meiosis I (as in Pelodera strongyloides, Section 4.1) (Figure 3C). Yet the anaphase migration of the $\mathrm{X}$ chromosomes was delayed compared to that of the autosomes-so much so that in some cases, both chromatids were found in the same spermatocyte after the first division, in which case the equational segregation occurred at the second division (Nigon, 1949a).

\section{In today's light}

Studies of meiosis in C. elegans have largely developed in the last ten years (Rog and Dernburg, 2013; Lui and Colaiacovo, 2013). The dynamics of chromosome synapsis can be followed live (Rog and Dernburg, 2015). A single crossing-over occurs for each pair of homologous chromosomes in C. elegans meiosis (Meneely et al., 2002), usually on a chromosome arm rather than in the center of the chromosome; the site of the unique crossing-over defines a long and a short chromosomal arm. Specific protein complexes associate and regulate double-stranded breaks, crossovers, short and long arms of chromosomes, and apposed homologs versus chromatids (Rog and Dernburg, 2013; Lui and Colaiacovo, 2013). The V shape (Y shape in Nabeshima et al., 2005) is transient in early diplotene, when the long arms but not the short arms have separated. When the short arm chromatids separate, it becomes a tetrad (Chi or X shape; Nabeshima et al., 2005). Further condensation, especially of the short arm, results 
in the Phi shape described above, and finally a bivalent, where the long arms are located outside and will attach to the spindle microtubules. Chromosomes in $C$. elegans are holocentric, meaning that centromeres are diffuse and the microtubules contact a large part of the chromosome (Albertson et al., 1997; Albertson and Thomson, 1993; Schvarzstein et al., 2010; Melters et al., 2012).

\subsection{The X-chromosome-to-autosome ratio in sex determination: use of a tetraploid line}

\subsubsection{A tetraploid animal line}

After heat shock of C. elegans, some of the progeny were of larger size than usual (Nigon, 1949b; Nigon, 1951a). A cytological study showed that these animals were tetraploids. They were self-fertile and their progeny were highly variable in several respects: there were individuals of diverse sizes, hermaphrodites (fertile or sterile) and males, intersexes, arrested larvae, etc.; some reverted to diploids, and in one such case, some animals resembled the $C$. briggsae micro mutant (Section 9.2); this Micro phenotype (Dumpy) could not be stabilized.

This was an unprecedented experiment. In the animal kingdom, fertile lines of induced tetraploids had not been obtained so far. A further aim was to know how long and under which conditions this polyploid form could be maintained. Starting from a population with variable phenotypes, Nigon applied a simple selection, based on size and fecundity. After about 20 generations, variability was lowered. During this first phase, unknown genome changes may have occurred. But variant forms reappeared at each generation and the maintenance of polyploids still required selection to eliminate them. Tetraploidy could be maintained for 78 generations, after which the culture was lost accidently. Nigon decided not to start again, lacking appropriate means to study this interesting variable phenomenon.

The male frequencies in the progeny of 426 tetraploid hermaphrodites displayed a bimodal distribution (Figure 5). The left side of the distribution corresponded to a class of individuals producing few males; this class was called thelygenous (producing females). The right side of the graph revealed a class of individuals with a higher and more variable male proportion; this class was called allelogenous (producing the alternative form).

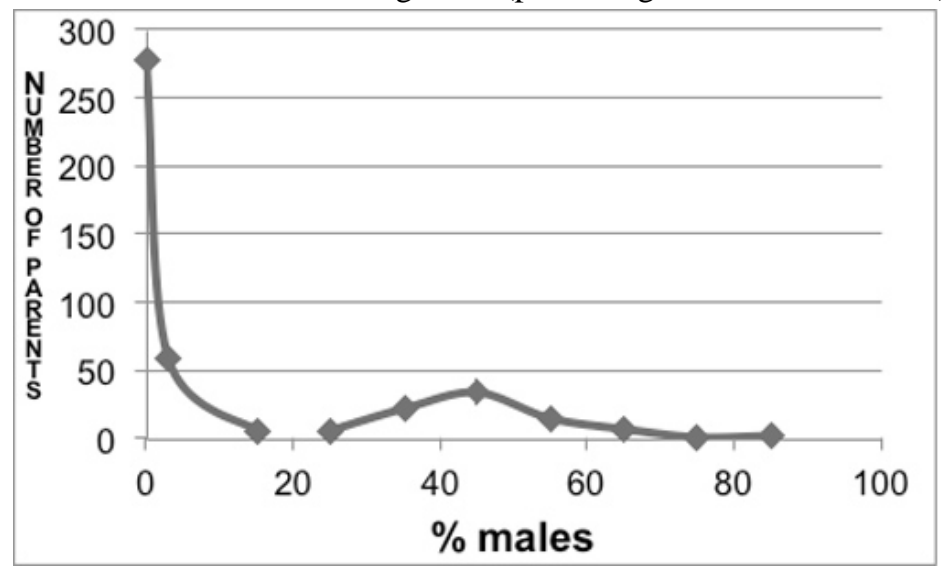

Figure 5. Distribution of male frequency in the progeny of $\mathbf{4 2 6}$ hermaphrodites of large body size in the tetraploid line. From Nigon (1951a) (removing animals with $<5$ progeny).

Further experiments showed that thelygenous parents could give rise to both thelygenous and allelogenous progeny, the latter in small proportions. Allelogenous parents could also give rise to both types, but with a higher proportion of allelogenous progeny (and of males) (Table 1). From one thelygenous individual and one allelogenous individual, two divergent lines were established. At each generation, the parents of the next generation were selected in the corresponding class, thelygenous in one line, allelogenous in the other. 30 generations of this treatment did not yield any differences in the stability of either line; the total of the counts are presented in Table 1 . Note that the allelogenous individuals also produced sterile eggs that were ignored; thus, the proportion of sexes may not correspond to that of the eggs. As a reference, the diploid line produced approximately $0.2 \%$ males. 
Table 1. Observed proportions of progeny types in thelygenous and allelogenous lines of C. elegans tetraploids. From Nigon (1951a).

\begin{tabular}{|l|l|l|l|}
\hline Line & \% Thelygenous & \% Allelogenous & Males \\
\hline Thelygenous & 92.7 & 6.7 & 0.6 \\
\hline Allelogenous & 17 & 41 & 42 \\
\hline
\end{tabular}

The average length of thelygenous hermaphrodites was $1560 \mu \mathrm{m}$, compared with $1300 \mu \mathrm{m}$ for diploids; the average length of allelogenous hermaphrodites was $1360 \mu \mathrm{m}$, and that of tetraploid males of $1150 \mu \mathrm{m}(1000 \mu \mathrm{m}$ for diploid males). Eggs had a volume of $43.10^{3} \mu \mathrm{m}^{3}$ for diploids, $62.10^{3} \mu \mathrm{m}^{3}$ for thelygenous tetraploids, and $55.10^{3}$ $\mu^{3}$ for allelogenous tetraploids. Ratios between the respective volumes of body and egg sizes were thus similar for thelygenous and allelogenous hermaphrodites, suggesting that the adult size difference may result from cell size differences. The mean brood size of thelygenous hermaphrodites was 21 -versus 260 in the diploid form. The fecundity of allelogenous hermaphrodites was further reduced, because they laid a higher proportion of dead eggs than thelygenous animals.

\subsubsection{Sex determination in the tetraploid line: hypothesis of the X-chromosome-to-autosome ratio}

A cytological analysis was performed on 74 individuals of the thelygenous and allelogenous lines (Figure 6; Table 2). Because of the arrested embryos and the small number of tested individuals, the numerical results must be treated with caution. However, the proportion of animals examined for their cytology was compatible with the proportions of thelygenous and allelogenous progeny in Table 1.

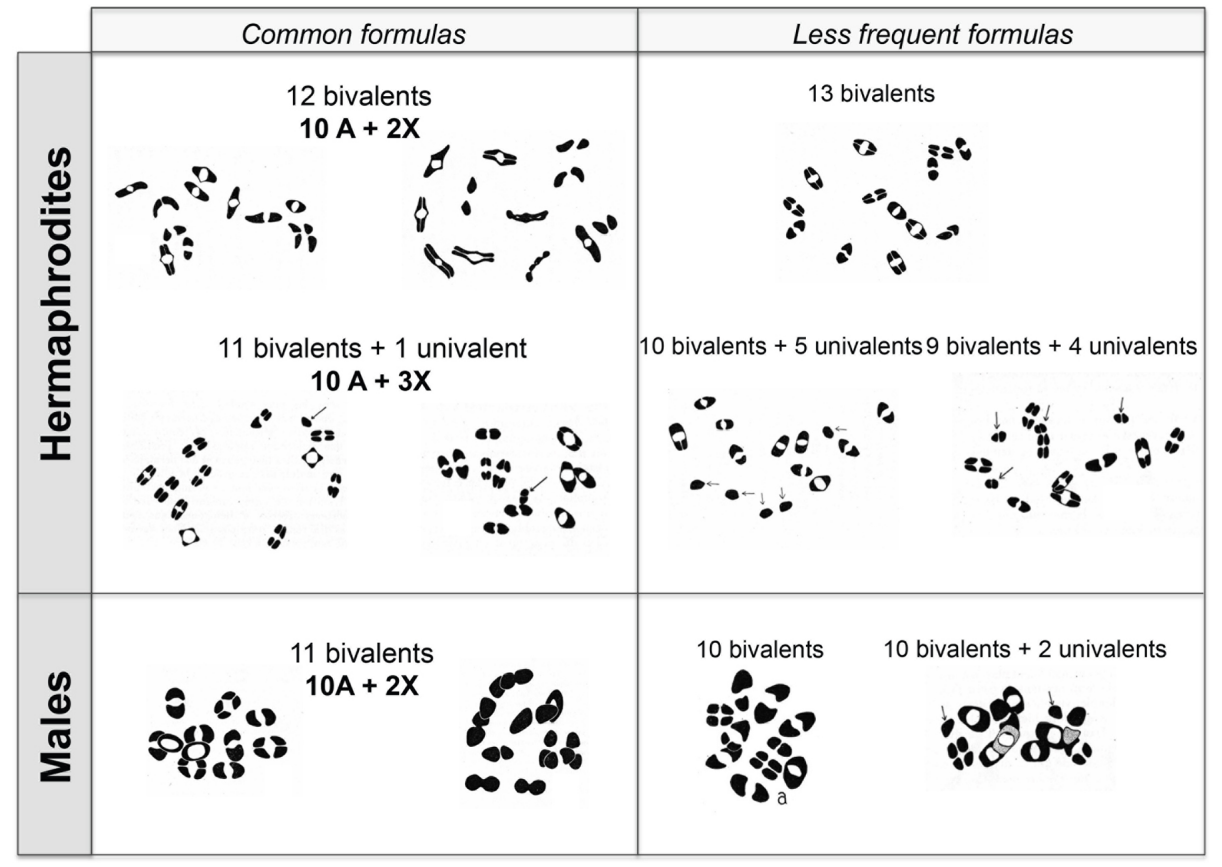

Figure 6. Chromosomal content of tetraploids. Modified from Nigon (1951a). Drawings of diakinesis stages. The most frequent 'regular' formulas are displayed on the left, the rare ones on the right. Arrows point to univalents. 
Table 2. Chromosomal counts in C. elegans tetraploid forms. $\mathrm{Al}=$ Allelogenous, $\mathrm{Th}=$ thelygenous. The most frequent chromosomal formulas are indicated in bold. From Nigon (1951a).

\begin{tabular}{|c|c|c|c|c|}
\hline $\begin{array}{l}\text { Structures in } \\
\text { diakinesis }\end{array}$ & Th line herm. & Al line herm. & Al line males & Interpretation \\
\hline 13 bivalents & 1 & & & $4 A+6 X$ \\
\hline $\begin{array}{c}12 \text { bivalents }+1 \\
\text { univalent }\end{array}$ & 2 & 1 & & $4 A+5 X$ \\
\hline 12 bivalents & 24 & 8 & & $4 A+4 X$ \\
\hline $\begin{array}{c}11 \text { bivalents }+4 \\
\text { univalents }\end{array}$ & 1 & & & $4 A+6 X$ \\
\hline $\begin{array}{l}11 \text { bivalents }+2 \\
\text { univalents }\end{array}$ & & 1 & & $4 A+4 X$ \\
\hline $\begin{array}{c}11 \text { bivalents + } 1 \\
\text { univalent }\end{array}$ & 1 & 14 & & $4 A+3 X$ \\
\hline 11 bivalents & 1 & 1 & 10 & $4 \mathrm{~A}+2 \mathrm{X}$ \\
\hline $\begin{array}{c}10 \text { bivalents }+2 \\
\text { univalents }\end{array}$ & & 1 & & \\
\hline $\begin{array}{c}10 \text { bivalents }+1 \\
\text { univalent }\end{array}$ & & & 3 & $4 A+1 X$ \\
\hline 10 bivalents & & & 3 & \\
\hline $\begin{array}{c}9 \text { bivalents }+4 \\
\text { univalents }\end{array}$ & & 1 & & \\
\hline $\begin{array}{c}9 \text { bivalents }+1 \\
\text { univalent }\end{array}$ & & & 1 & \\
\hline Total of individuals & 30 & 27 & 17 & \\
\hline
\end{tabular}

Supposing that univalents were most often unpaired $\mathrm{X}$ chromosomes, one could infer that the hermaphrodite, of female morphology, could be obtained with the standard tetraploid formula $4 \mathrm{~A}+4 \mathrm{X}$ (where A stands for the five autosomes), as well as with $4 \mathrm{~A}+3 \mathrm{X}, 4 \mathrm{~A}+5 \mathrm{X}$ and $4 \mathrm{~A}+6 \mathrm{X}$. The chromosomal formula $4 \mathrm{~A}+1 \mathrm{X}$ gave rise to males. The formula with 11 bivalents (which can be interpreted as $4 \mathrm{~A}+2 \mathrm{X}$ ) usually gave rise to males, but might also give rise to hermaphrodites, likely because the abnormality concerned another chromosome.

These results suggested that the balance of $X$ chromosomes to autosomes (X:A) determined sex, with the $X$ chromosome exerting a feminizing influence and the autosomes a masculinizing effect. In the normal hermaphrodite, the X:A ratio is 1 , in the normal male 0.5. Tetraploids allowed intermediate values: individuals with a X:A ratio of 0.75 developed as fertile hermaphrodites. Some animals developed as intersex males. The critical X:A ratio was thus between 0.5 and 0.75 , with some intersexes in the intermediate range (Nigon, 1951a).

The observations concerning the different forms of reproduction and sex determination led Nigon to discuss hypotheses concerning the mechanisms of sex determination (Nigon, 1951a). We will limit ourselves here to two main directions:

1) The activity of the $X$ chromosome seemed to play an important role. However, the cytological identification of the sexual chromosome often remained impossible. The study of tetraploids and various abnormalities induced by high temperature further suggested that the sex chromosome acted in combination with other factors, some of which were carried by autosomes.

2) The link between sex determination in the germ line and in the soma was not absolute, based on the fact that in C. elegans, one could find hermaphroditic gonads in male morphologies as well as in female morphologies. Put another way, the factors that determine the somatic morphology could be separated from those leading to the sexual differentiation of gametes. 


\section{In today's light}

We saw above that Nigon solved the problems of crosses by developing an adequate and rigorous culture method. The choice of an appropriate cytological method (Section 2.2) was the result of the same methodological rigor. Most importantly, these cytological studies allowed Nigon to identify the chromosomal basis of sex determination in C. elegans.

On X non-disjunction: Nigon observed that the spontaneous males obtained from hermaphrodites were more often sterile than the males obtained during further generations of crosses. The partial sterility of the spontaneous males may be attributed to autosomal abnormalities occurring concomitantly with X-chromosome non-disjunction. These other chromosomal abnormalities may lead to sterility and thus be naturally eliminated in the following generations of crosses. Mutants with increased X-chromosome non-disjunction (affecting chromosome pairing and synapsis) have since been found by screening for a high incidence of males (him) in the progeny (Hodgkin et al., 1979). XXX animals that are produced as a counterpoint to X0 males are dumpy (short) and likely explain the unstable short animals produced by the tetraploid line. Most mutants with a high incidence of males also affect autosomes and thereby result in a high proportion of dead, aneuploid progeny. The X chromosome has, however, a specific pairing mechanism and its non-disjunction is specifically increased in some mutants (Hodgkin et al., 1979; Phillips et al., 2005). Whether most spontaneous non-disjunction of X chromosomes in C. elegans hermaphrodites occurs in oogenesis (reduction division) (Hodgkin et al., 1979) or spermatogenesis (Meneely et al., 2002) still seems unclear. Reduction and lagging of the $\mathrm{X}$ chromosome during the first meiotic division in $C$. elegans male spermatogenesis has been observed by Shakes et al. (2009).

On sex determination: Nigon established that $C$. elegans used a XX/X0 sex determination mechanism. How is the $\mathrm{X}$ chromosome counted? One important aspect of the chromosomal basis of sex determination was Nigon's analysis of the tetraploid line. By using a combination of diploids and tetraploids in C. elegans, he correctly inferred that the sex of the individual was determined by the ratio between sex chromosomes and the other chromosomes: the autosomes were participating in sex determination, as the denominator. Nigon's inference has since been validated and further refined.

A tetraploid line was later obtained from C. elegans Bristol after heat-shock by Madl and Herman (1979). This line included autosomal and X-linked marker mutations and is available at the Caenorhabditis Genetics Center (CGC). Like Nigon, Madl and Herman found two types of hermaphrodites, which they called LFM (low frequency of males, thelygenous; 4A+4X) and HFM (high frequency of males, allelogenous; $4 \mathrm{~A}+3 \mathrm{X}$ ), with unclassifiable individuals. Madl and Herman (1979) confirmed the existence of diverse chromosomal formulas, also suggesting the presence of autosomal aneuploids. In addition, they formed triploid individuals by crossing tetraploids and diploids and found that $3 \mathrm{~A}+2 \mathrm{X}$ animals are males. They thus refined the critical ratio of $\mathrm{X}$ : A as lying between 0.67 and 0.75 .

Partial X-chromosome duplications further suggested that multiple loci on the $\mathrm{X}$ chromosome act in feminization (Madl and Herman, 1979). Several elements on the X chromosome and on the autosomes have now been identified as being involved in this counting, thus proving the validity of the X:A ratio in determining sex (Somatic sex determination).

In addition, since variability and intersexes were observed, Nigon concluded that some other factors must be at play. This variable expressivity likely corresponds to variation in activation of some components of the sex determination pathway in different tissues of an individual.

On tetraploidy: Nigon's tetraploid line corresponds to the first experimental induction and maintenance of tetraploidy in an animal (to our knowledge). The instability of the tetraploid situation in meiosis was noted by the later authors (Madl and Herman, 1979; Meneely, 1994) but it was not further studied. Possibly, some chromosomal combinations are viable but display abnormalities. This variability of the progeny of tetraploids is likely intrinsic to the situation of a meiotic prophase with four homologs, which might induce a structural variability that is difficult to stabilize. Are plant tetraploids perhaps more stable due to a difference in the mechanism of meiosis? An alternative is that self-fertilization may favor tetraploidy (Barringer, 2007) and is more common in plants.

A recent review on variations induced by polyploidy can be found in Wertheim et al. (2013). A key problem is the dosage of gene expression in the presence of abnormal contents of chromosomes. In C. elegans diploids, it is now known that if any of the autosomes is haploid, the change in dosage results in lethality (Hodgkin et al., 1979). For the $\mathrm{X}$ chromosome, specific chromosomal dosage compensation mechanisms ensure a similar level of expression in hermaphrodites and males (X-Chromosome dosage compensation). 


\section{Parthenogenesis}

Some free-living nematode species reproduce via parthenogenesis, without true fertilization. Parthenogenesis occurs in two main modes, depending on whether sperm is involved, and each mode can be further subdivided.

\subsection{True parthenogenesis}

In true parthenogenesis, no sperm is involved. Among the species studied by Maupas (1900), this mode of reproduction was found in Rhabditophanes schneideri, Plectus cirratus, and Aphelenchus agricola, as well as four new species he described: Cephalobus dubius, Cephalobus lentus, Alaimus thamugadi, and Macrolaimus crucis (Maupas, 1900). Maupas noted the absence of sperm in the ovary and the absence of males, which made it likely that these species were true parthenogens. He did not further study the cytology of meiosis.

In the animal kingdom, two subtypes of true parthenogenesis have been described, depending on the presence of chromosome pairing. In both cases, the oocyte nucleus undergoes a single maturation division and generally emits a single polar body. In the first subtype, the chromosomes are paired during oogenesis, and recombination can occur between them. In the second subtype, pairing does not occur, and the single maturation division is equational, but apparently without recombination. Both meiotic and mitotic parthenogeneses have been described in plant parasitic nematodes, such as Meloidogyne spp. (Triantaphyllou and Hirschmann, 1964). Karl Belar (see Section 18.3) described the cytology of meiosis in free-living nematodes with true parthenogenesis, using a single, undescribed, species, Rhabditis XIX (Figure 7). This species displayed diakinesis and pairing, followed by separation of sister chromatids after anaphase. Here, in contrast to usual female meiosis, focused centrosomes remained during meiosis (thus alleviating the need for a spermatozoon to provide them) (Belar, 1923; Belar, 1924).

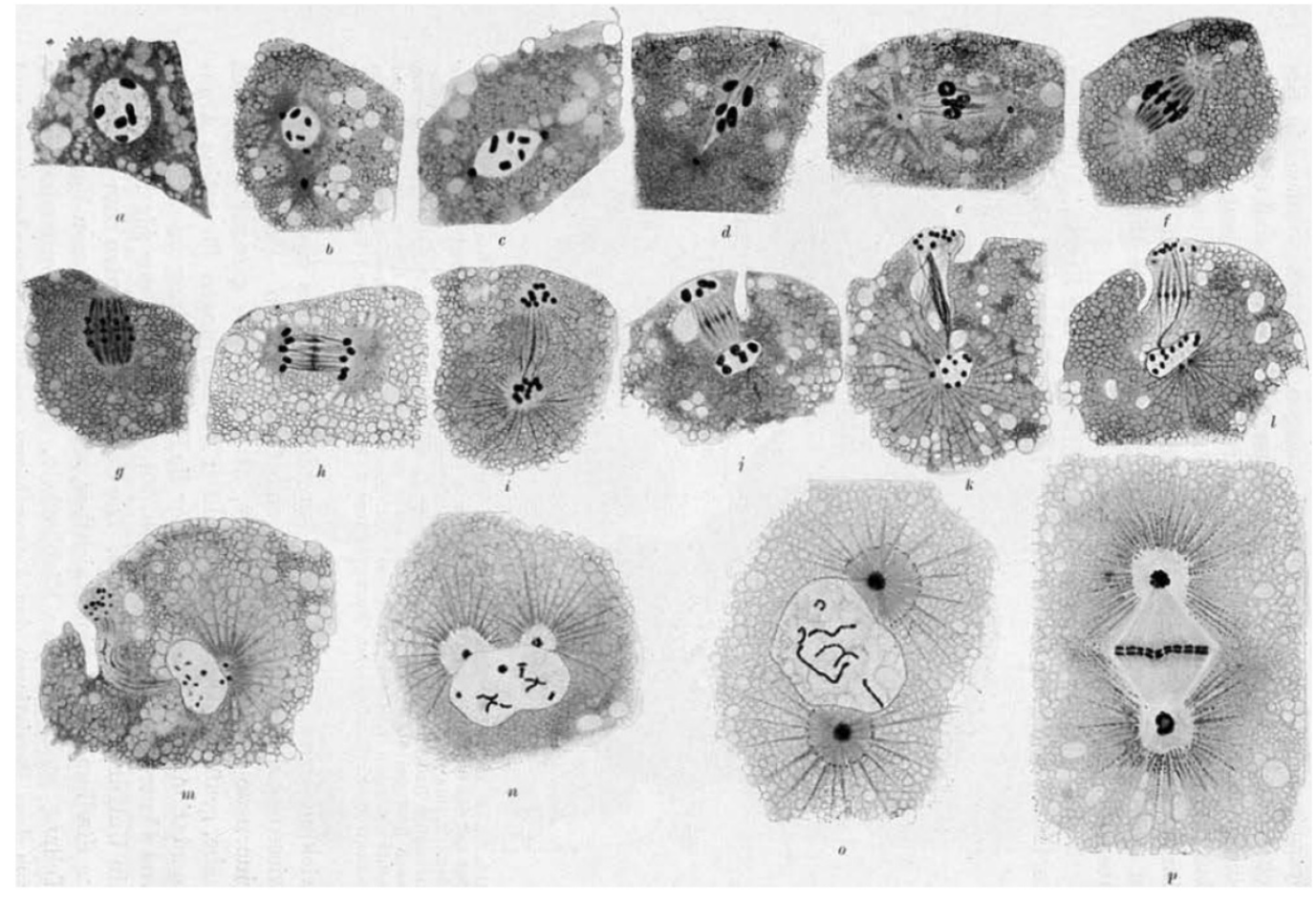

Figure 7. Meiosis and first embryonic cleavage in parthenogenetic Rhabditis XIX. From Belar (1924), with permission from Springer Publishing Company. (a-p) Successive stages are shown. Fixed eggs, hematoxylin-stained paraffin sections. The oocyte centrosome is visible in panel (b), and divides once during oogenesis (e). No polar body is formed and the corresponding chromosomes degenerate in the egg cytoplasm (m). The first embryonic cleavage spindle develops along the egg long axis (n-p). The illustrations are drawings by Belar.

\subsection{Pseudogamy}

The second major mode of parthenogenesis requires a spermatozoon to activate the oocyte. The sperm chromosomal material then degenerates without contributing to the genome of the progeny. This type of parthenogenesis, known as merospermy or pseudogamy, can be further divided into three subtypes. 


\subsubsection{Pseudogamy with hermaphroditism: Rhabditis aberrans}

The first subtype was described by Eva Krüger in a species she named Rhabditis aberrans (Krüger, 1913). This species was composed of somatic females producing sperm (apparent protandric hermaphroditism) and a particularly low proportion of males ( 0.4 males for 1000 hermaphrodites). The sperm could be seen to enter the oocyte before the onset of the oocyte maturation division and then rapidly degenerated. During the unique maturation division of the oocyte, a diploid stock of 18 chromosomes could be observed, without pairing. At anaphase, the sister chromatids of the 18 unpaired chromosomes separated and a single polar body was expelled. No second maturation division ensued. The diploid oocyte nucleus then moved towards the egg center, and the first embryonic cleavage took place. In a single case, the author observed on living material the extrusion of two polar bodies and the presence of two pronuclei. This possible true fertilization suggested an alternative between parthenogenetic and amphimictic development, as described below for Mesorhabditis (Section 5.2.3) and Strongyloides spp. (Section 8.2).

Spermatogenesis in $R$. aberrans hermaphrodites unfolded quite differently from oogenesis and overall in a standard fashion. At diakinesis, 8 bivalents and 2 univalent chromosomes (likely sex chromosomes) could be observed. On one occasion, the author observed 9 bivalents. The first division was reductional for the bivalents, and equational for the univalents. At the second division, these previously univalent chromosomes formed a bivalent and underwent a reduction division. In some cases, however, this bivalent separated with a delay and a spermatid with 8 chromosomes and one with 10 were produced. This bivalent could also be lost at metaphase. Thus, spermatogenesis appeared normal, albeit with some exceptions.

Males could result from an abnormal maturation division of the oocyte or from an amphimictic fertilization by a sperm already bearing a chromosomal abnormality, such as X0. In August 1911, Krüger obtained males at an average rate of $1.6 \%$ in a group of six cultures, which lasted for only one generation, while other cultures at the same time produced males in the usual proportions. This high male frequency may have been the consequence of a higher temperature.

Krüger agreed with Maupas that the ability to reproduce without males was likely evolutionarily derived from a situation with males and females. In natural settings, $R$. aberrans, like many free-living nematodes, went through periods of abundant food and fasting. Krüger suggested that hermaphroditism and parthenogenesis might be advantageous in such circumstances because a single individual was able to reproduce.

A pseudogamous hermaphroditic species he called Rhabditis XX was further observed by Belar (1923). In this species as well, meiosis occurred differently in spermatogenesis versus oogenesis. In this species, only partial chromosome pairing was observed during spermatogenesis.

\subsubsection{A pseudogamous mutant of Pellioditis pellio}

Paula Hertwig (see Appendix 3, Section 18.2) described the appearance of parthenogenesis as a "mutation" in a gonochoristic species, Rhabditis pellio (now Pellioditis pellio) (Hertwig, 1920). In January 1916, she isolated a male and a female of $P$. pellio from decomposing earthworms. This pair gave birth to an approximately equal number of male and female progeny. At some point, the progeny from this single pair was divided into two cultures. At a later date, the author noticed that in one culture, the population was composed of males and females in similar proportions, while, in the other, the population now contained mostly females. Five days later, she isolated a few unfertilized young females from this male-poor culture and added three males from the male-rich culture. These females produced only $12 \%$ males in their progeny, many of them with abnormal morphology. Hertwig showed that the same male individual from the male-rich culture could fertilize females of both cultures, giving rise to a 1:1 sex ratio or to a female-biased sex ratio, depending on the origin of the female. Male production continued to decrease in the male-poor culture. Finally, after an unspecified time, the females produced only female progeny. The mutant culture could then only be continued by addition of males from $R$. pellio. The conservation of this form thus required that both cultures be kept in parallel.

Cytological observations indicated that the male-poor culture reproduced via pseudogamy. Oogenesis and spermatogenesis of $P$. pellio normally took place with two standard maturation divisions, with pairing of 7 bivalents. In the pseudogamous form, the equatorial plate of the unique oocyte maturation division displayed a diploid number of 14 chromosomes, without pairing. Cytology did not suggest that the pseudogamous culture had undergone tetraploidization. The oocyte maturation division was triggered by entry of the sperm from the male. As in $R$. aberrans, the sperm did not participate in the formation of a fertilization nucleus in the pseudogamous form of $P$. pellio (Figure 8). 

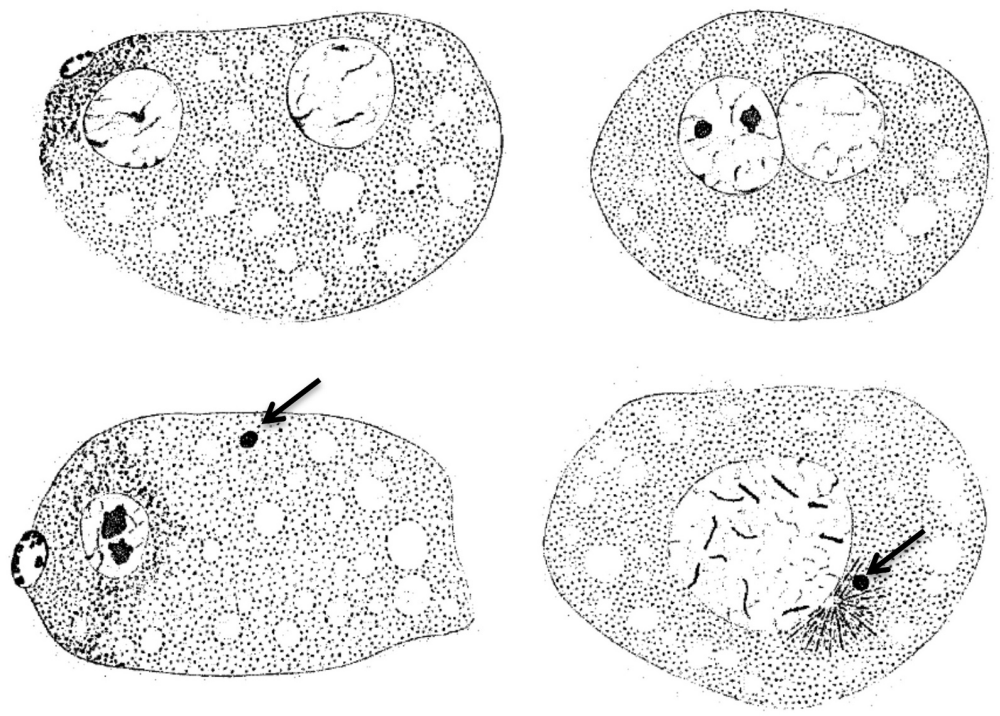

Figure 8. Wild-type (top) and pseudogamous (bottom) form of $\boldsymbol{P}$. pellio after fertilization and oocyte meiosis, at two successive stages. Drawings, from Hertwig (1920). The oocyte nucleus and polar body are to the left, the sperm nucleus to the right. While two pronuclei (female and male) are formed in the wild form, the sperm nucleus stays condensed and does not participate to the development of the pseudogamous form (arrow).

To demonstrate the absence of genetic contribution from the sperm in this strain, Hertwig performed a further experiment: she irradiated males with radium so as to inactivate their genetic material. These males remained able for several hours after irradiation to mate and provide sperm, but the irradiated sperm chromosomes were degraded in the egg. If the females came from the normal form, the eggs did not develop past the few first cleavage divisions. If the females were from the pseudogamous line, the eggs developed normally. This demonstrated that, in the latter line, the genetic material from the male was not required for development. This uncoupled the two roles of sperm, on one hand in triggering oocyte meiosis and subsequent embryonic development, and on the other hand in providing genetic material.

After collecting further nematodes in earthworms, Hertwig reported on finding pseudogamous forms of three species in nature. She gave the name Rhabditis anomala to the natural pseudogamous species, stating that it might be equivalent to R. aberrans from Eva Krüger (Hertwig, 1920; Hertwig, 1922).

\subsubsection{Pseudogamous gonochorism with partial true fertilization: Mesorhabditis spp.}

A third form of pseudogamy was discovered by Belar (Belar, 1923; Belar, 1924). In this case, the cultures contained 5 to $20 \%$ males. From live observations, Belar noticed two types of eggs. In the first type, the sperm chromosomes that had been provided by the male degenerated in the egg (Figure 9). In the vicinity of the degenerating sperm nucleus, were two centrosomes, from which asters grew. Belar used oxygen depletion to slow down movements in the cytoplasm and better visualize the growing asters and the origin of the centrosomes. The asters could thus be clearly seen as originating from the region where the sperm entered. The female pronucleus moved towards them and these asters formed the first mitotic spindle. The second type of egg underwent two oocyte maturation divisions; a male pronucleus was formed and fused with the female pronucleus, following the course of usual meiosis and fertilization. This species thus used both pseudogamous and amphimictic reproduction. In the pseudogamous form, the male sperm did not contribute genetic material, but brought the centrosome. Belar suggested that the amphimictic eggs gave rise to males. 
A

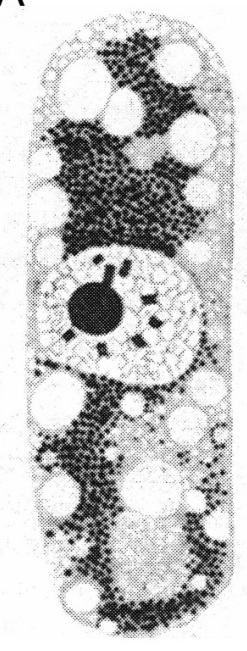

D

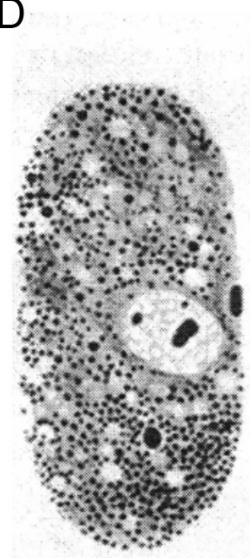

B

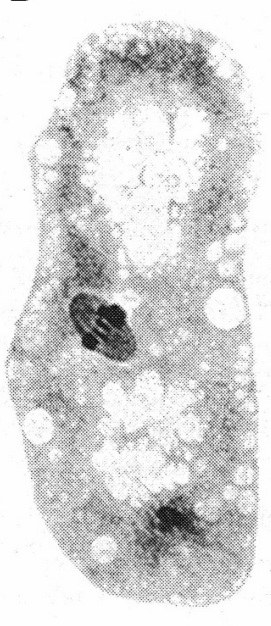

E

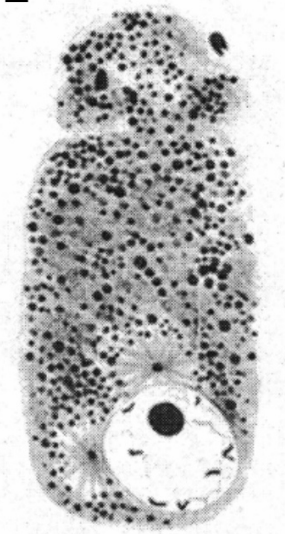

$\mathrm{C}$

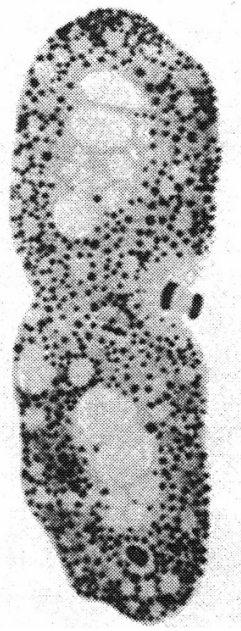

$\mathrm{F}$

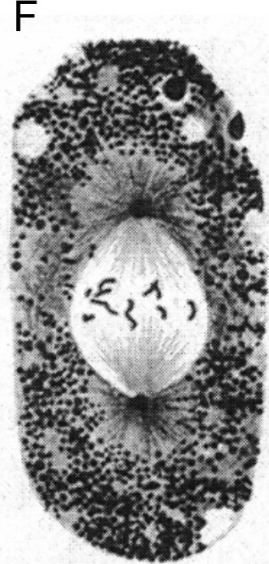

Figure 9. Pseudogamous fertilization in Mesorhabditis sp. Modified from Belar (1924), with permission from Springer Publishing Company. The top panels are rotated in the original. Fixed eggs, hematoxylin-stained paraffin sections. The pole oriented towards the vulva (posterior pole) is at the bottom. (A) Unfertilized oocyte. (B) The sperm has entered at the bottom pole, and the oocyte nucleus enters meiosis. (C) Anaphase of oocyte meiosis. (D) Oocyte nucleus reformation. During these stages, the sperm nucleus has remained condensed at the posterior pole (black oval). (E) Asters then form at the posterior pole, and the female pronucleus moves towards them. (F) First cleavage spindle.

Belar, with the help of Micoletzky, identified the species as Rhabditis monhystera Bütschli, whose males were at the time of Bütschli unknown. Nigon (1949a) studied the morphology of both sexes in a species collected from nature, where the females were morphologically similar but not identical to $R$. monhystera Bütschli. This species displayed males, like that used by Belar. Nigon thus considered that both his and Belar's culture were a distinct species from that of Bütschli, and named it Rhabditis belari (now Mesorhabditis belari).

Nigon further studied M. belari using experiments and cytological observations (Nigon, 1947; Nigon, 1949a). Spermatogenesis was difficult to follow in males because of the small cell size and the high chromosome number, yet overall appeared similar to that of a normal gonochoristic species. 10 pairs of chromosomes were observed at diakinesis. One pair contained a chromosome that was larger than all others, possibly a Y chromosome. The first division was reductional. At the second division, 10 chromosomes moved in a synchronous manner. The large chromosome was found in some spermatocytes.

In amphimictic eggs of M. belari with two pronuclei, Nigon observed that two polar bodies were emitted at the equator. The female pronucleus then appeared next to them, while the sperm nucleus appeared at one pole (Figure 10). Such eggs, with two pronuclei, were fixed under the microscope at the time of pronuclear meeting. Staining showed that each pronucleus contained a haploid set of chromosomes. These oocytes had undergone a normal maturation with reduction. 
In pseudogamous eggs instead, Nigon observed that oogenesis conformed to the equational scheme described by Belar, while the sperm genetic material degenerated. In most pseudogamous eggs, the oocyte chromosomes formed a metaphase circle of 10 chromosome pairs. The sister chromatids, already dissociated during anaphase, yielding 20 chromosomes. More rarely, a metaphase plate with 20 unpaired chromosomes formed directly (Figure 10). A single polar body was emitted. As suggested by Darlington (1937), the timing of tetrad separation seemed the key factor that distinguished pseudogamous from amphimictic oocytes.

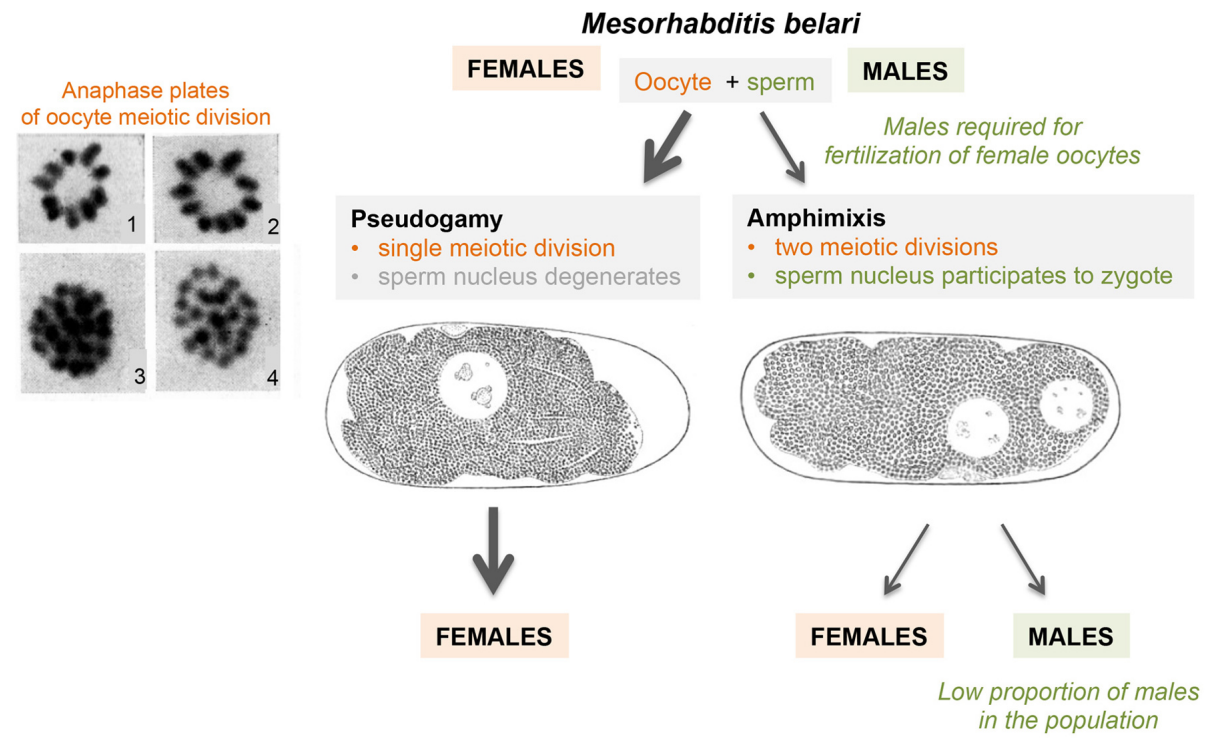

Figure 10. Modes of fertilization of Mesorhabditis belari and their relationship with sex determination. The drawings of the pseudogamous and amphimictic eggs are from Belar (1924), with permission from Springer Publishing Company. The pictures of chromosomes are from Nigon (1949a), showing two subtypes of meiotic division in pseudogamous eggs: (1) and (2) are anaphase plates where 10 paired bivalents form a circle at metaphase and are reduced in the unique maturation division, (3) and (4) are two anaphase plates of a pseudogamous egg where the metaphase plate contained 20 unpaired chromosomes.

Nigon followed fertilization in live eggs and could separate those showing one or two pronuclei. Out of 99 isolated eggs, 80 developed with a single pronucleus and 19 with two. From 25 eggs of the former class, development was followed until the adult stage, which was reached for 19 of them, giving rise to 19 females. In contrast, 10 amphimictic eggs with two pronuclei gave rise to 7 males and 3 females (which may correspond to a 1:1 ratio determined by chromosomal content). Thus, contrary to Belar's supposition, the amphimictic eggs gave rise to both males and females, whereas the parthenogenetic ones only gave rise to females. From the asymmetric size of one chromosome pair in the males, sex determination seems to follow a XX/XY scheme.

To determine the proportion of sexes, 11 male-female pairs were isolated and gave rise to a total of 631 males and 5559 females, thus a mean of $10.2 \%$ males (range 3-17\%). In another experiment, pairs were isolated and transferred every two days in order to examine variation as a function of age, and the cultures were maintained at four temperatures ranging from $13-25^{\circ} \mathrm{C}$. At all temperatures, the proportion of males decreased progressively with maternal age, from 19 to $6 \%$ at $13{ }^{\circ} \mathrm{C}$, from 13 to $3 \%$ at $17{ }^{\circ} \mathrm{C}$ and beyond. Addition of old medium decreased the male frequency from 19 to 12 . These variations in sex ratio likely corresponded to variations in the proportion of amphimictic eggs. In summary, in this species, the amphimictic or parthenogenetic nature of reproduction appeared to depend on oocyte constitution, itself influenced by age, temperature and culture medium (Nigon, 1949a; Nigon, 1952). The sperm nucleus behavior then correlated with that of the oocyte nucleus.

\section{In today's light}

The variety of reproductive modes of free-living nematodes has great potential for studies on the evolution and impact of reproductive modes. Pseudogamy is found in other organisms (Beukeboom and Vrijenhoek, 1998), but has not been studied in nematodes since the work reported above.

We are not aware of any species in present culture with the pseudogamous hermaphrodite mode of reproduction described for $R$. aberrans, except perhaps Protorhabditis sp. JB122, which develops parthenogenetically (Brauchle et al., 2009), yet the females make sperm (M.-A. Félix, unpublished observations). In 
addition, pseudogamy may simply have been overlooked in species labeled "hermaphroditic" in culture collections. The modalities of gamete maturation are widely different in oogenesis and spermatogenesis of the same hermaphroditic $R$. aberrans individual. How meiosis is regulated differently in the two types of gamete remains unknown.

The observation by Hertwig (1920) of a "mutation" of the gonochoristic P. pellio into a pseudogamous form is intriguing, especially the fact that the mutation seems to have a correlated effect on female meiosis and sperm degeneration. The transformation appeared progressively over generations, perhaps through stabilization of an abnormal chromosome content or fixation of new or pre-existing mutations in the culture. These experiments would need to be reproduced on the same species or another, either by further sampling of $P$. pellio or by using induced mutagenesis or heat-shock. Whether this phenomenon is relevant in nature also remains to be explored.

Mesorhabditis belari, with its alternative mode of egg development, is particularly intriguing. Among species presently in culture, Mesorhabditis sp. PS1179 displays this mode of reproduction, which allows one to study the regulation of pseudogamy versus true fertilization in a given individual (M. Delattre and M.-A. Félix, unpublished).

Even true parthenogenetic free-living nematodes have been little studied. True parthenogens are found in genera in the sister clade to Caenorhabditis, such as Diploscapter (Brauchle et al., 2009), the Panagrolaimus, Halicephalobus, Rhabditophanes and Cephalobus/Acrobeloides genera (Cephalobina suborder), and aquatic species such as Plectus sp. The species Diploscapter coronatus (Rhabditidae) contains a single chromosome pair, which undergoes a single meiotic division without pairing (although the polar body later divided; Hechler, 1968; Lahl et al., 2006). In Acrobeloides nanus (Cephalobidae), a second meiotic division was observed but the two daughter cells then fused. Plectus sp. ES601 (Plectidae) produces two polar bodies; whether diploidy is achieved in this species through an additional round of DNA replication is unclear (Lahl et al., 2006). A better understanding of genetic segregation in parthenogens has been only achieved in some plant parasitic nematodes, such as Meloidogyne hapla (Liu et al., 2007).

Overall, this diversity of reproductive mode in free-living nematodes that are easy to culture in the laboratory opens up exciting possibilities of studies on their cell biological regulation and evolutionary context.

\section{Embryonic development}

The basis of our knowledge on descriptive and experimental embryology in nematodes derives mostly from studies of Parascaris equorum performed between 1890 and 1910. A synthesis can be found in Nigon (1965). The clear-sightedness of these early embryological studies, on par with those performed on sea urchins or amphibians then classical materials for embryologists, left far behind the few observations made on free-living nematodes, where the early embryonic divisions, as in Ascaris, separate cells of different potentials and fates.

\subsection{Asymmetry of the first division}

Nigon et al. (1960) observed the early stages of development on living embryos in several free-living species: $C$. elegans, $M$. belari, $R$. anomala, and $R$. maupasi. Using a camera mounted on the microscope, the behavior of the egg was recorded in time lapse. The first events following sperm entry had to be studied on eggs that were still in the female uterus, because at this stage the egg shell is under formation and does not sufficiently protect the embryo. Observations become easier later in development when the eggs can be isolated. The use of an incubator case for the microscope made it possible to vary the temperature (see Figure 22). Although embryo development was analyzed until the four-cell stage, we will here mostly report on events from sperm entry until first cleavage.

In free-living nematodes, the anatomy of the ovary and uterus made it possible to distinguish in the elongated shape of the mature oocyte a proximal or external pole, towards the vulva, and a distal or internal pole, towards the distal germ line. [These poles were called anterior and posterior, respectively, in the article by Nigon et al. (1960). Because the modern reader may be used to the reverse nomenclature, we use here proximal and distal in reference to the orientation in the gonad and restrict anterior and posterior to their current use, which corresponds to the anterio-posterior axis in embryonic development (animal-vegetal).] A key question concerned the appearance of cells of different developmental fates and the control of embryonic cleavage patterns during the early embryonic divisions. Each species was seen to elaborate specific variants on a background of mostly common events. In gonochoristic and hermaphroditic species, sperm entry occurred most often at the proximal pole (the future posterior embryonic pole). We will first describe the case of $C$. elegans in some detail, then examine the observed particularities of other species more briefly. 


\subsubsection{Observations on C. elegans}

In addition to triggering the oocyte maturation divisions, sperm entry was observed to trigger formation of the fertilization membrane, and later, of the egg shell. The egg cytoplasm retracted inside the egg shell. The surface of the cytoplasm changed its aspect continuously, forming bulges and grooves. The cytoplasm moved around in all directions. Movements appeared to be driven by modifications (contraction or expansion) that originated in a superficial structure at the level of the plasma membrane ( Nigon et al., 1960).
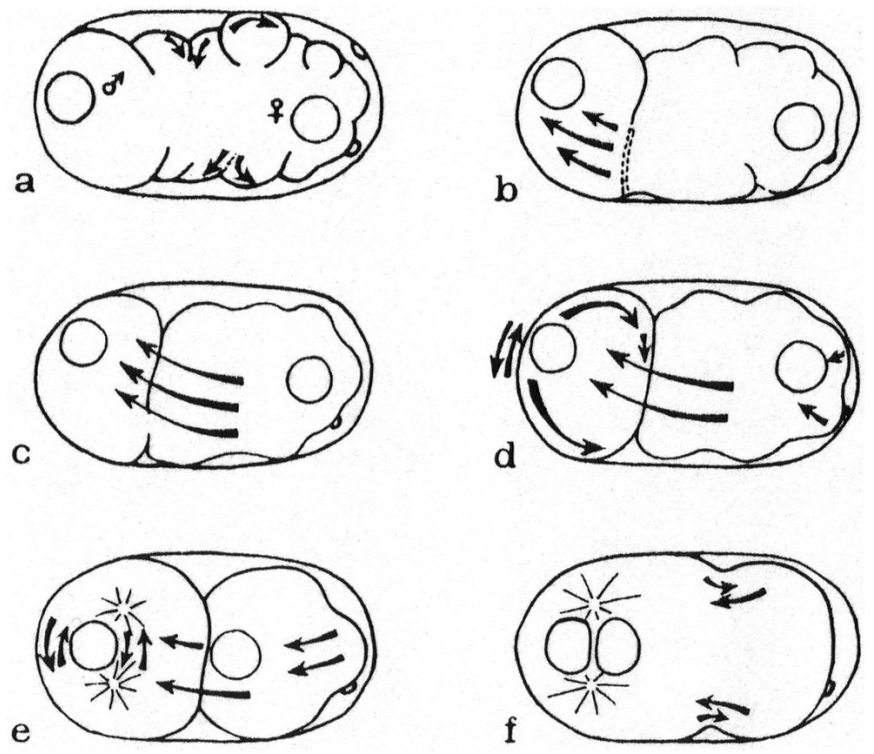

Figure 11. Local and global movements of the plasma membrane after fertilization in C. elegans. The two pronuclei and the pseudocleavage furrow are visible. The future embryonic posterior pole is on the left. Reproduced from Nigon et al. (1960).

At fertilization, the oocyte nuclear membrane was observed to break down, while the nucleus migrated slowly towards the anterior pole. The first maturation division then took place, and the egg shell started forming. About 10 minutes later, the plasma membrane became depressed at the anterior pole, where the second polar body formed. The female pronucleus appeared next to this polar body, while the male pronucleus appeared close to the posterior pole (Figure 11a). Many in-folds and protuberances were observed at the egg surface, while the cytoplasm underwent streaming. A strong constriction appeared at the posterior third of the egg, dividing the egg into two lobes. Because the egg seemed to be divided, this constriction was called a pseudomembrane (pseudocleavage; Figure 11). A constriction could be seen to start, retract, then was followed by another one. Finally, several such small contractions joined and surrounded the egg, and the pseudomembrane developed. The superficial deformations were at this time limited to the anterior part of the egg. The pseudomembrane moved towards the anterior pole, triggering a cytoplasmic stream from the anterior pole, flowing through the opening of the pseudomembrane towards the posterior pole. This flow dragged the female pronucleus towards the male pronucleus. The pseudomembrane started fading when it only encased at the anterior pole one third of the egg volume. Symmetrical figures of asters were observed to form on either side of the male pronucleus (Figure 11f). The asters then separated from each other, while the two pronuclei got closer to each other, towards the center of the egg. After nuclear envelope breakdown, the mitotic spindle formed along the egg long axis, with an asymmetric position towards the posterior pole. The asters then underwent four to nine oscillations (Figure 12a). The surface of the cytoplasm only displayed minimal movements at this time. 

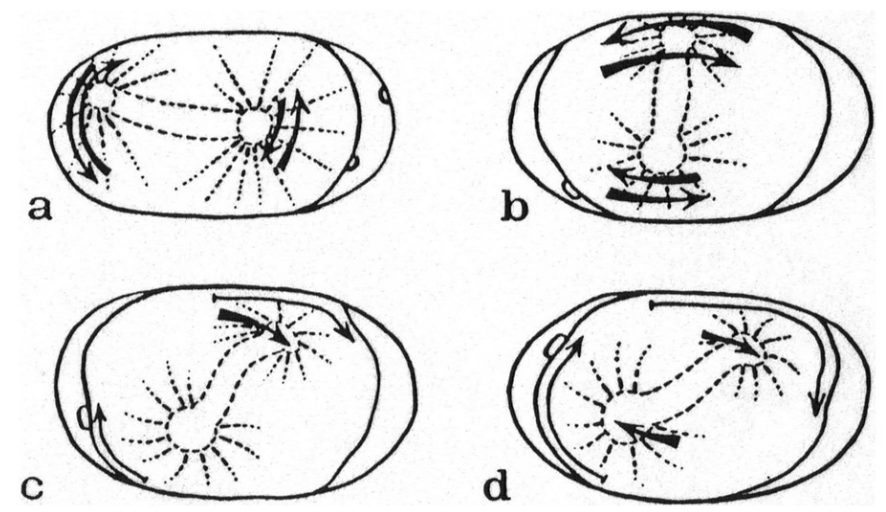

Figure 12. Mitotic spindle oscillations. (a) Oscillations of the first mitotic spindle in the fertilized egg of C. elegans. (b-d) Cortical rotation and spindle oscillations in $R$. anomala. The future embryonic posterior pole is on the left in (a), on the right in (b-d). Reproduced from Nigon et al., (1960).

Cytokinesis was observed to start with the formation of a shallow transverse furrow. This furrow formed at the spindle equator, located slightly posterior to the equator of the egg. A sharp incision progressively circled around the egg. Cytoplasmic granules in contact with the membrane were carried along with membrane elongation; using time-lapse movies, granule movement was seen to occur through successive waves. The egg division thus separated an animal cell at the anterior pole from a smaller vegetal cell at the posterior pole. The second division then occurred with a rotation of the spindle in the posterior blastomere (Figure 13).
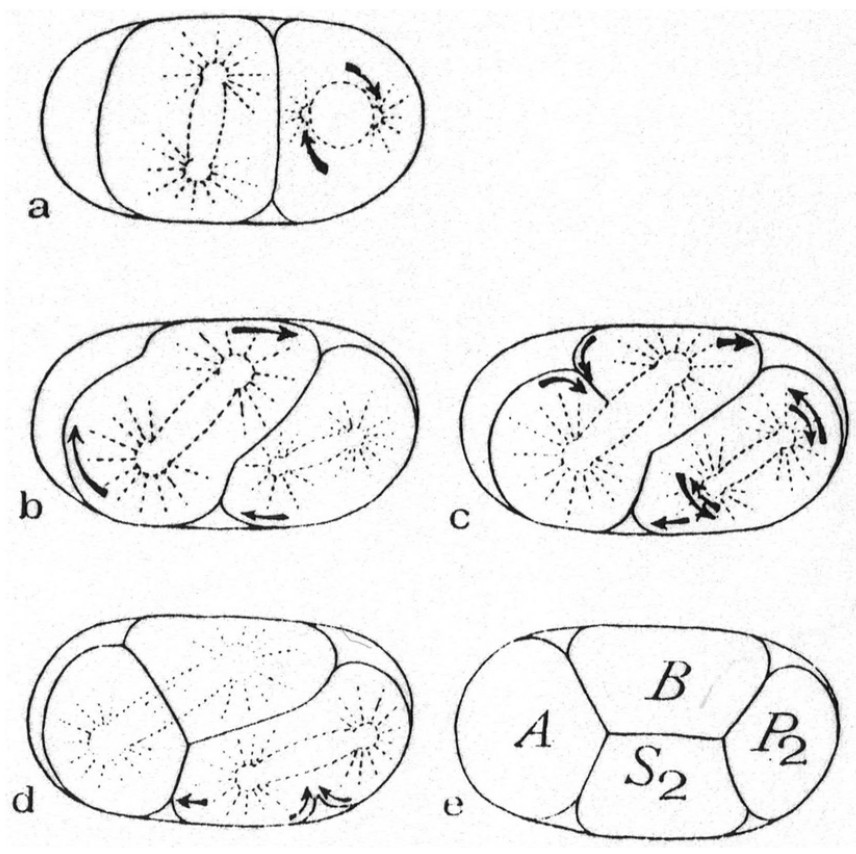

Figure 13. Second embryonic cleavage division in C. elegans. Reproduced from Nigon et al. (1960). The future embryonic posterior pole is on the right. The blastomeres A, B, S2, P2 (following Boveri's nomenclature) are now commonly called ABa, ABp, EMS and P2.

\subsubsection{Observations on Mesorhabditis belari}

In M. belari, the polar bodies were seen to be extruded in an equatorial position. The surface changes and the cytoplasmic streams occurred earlier in this species and were far more vigorous than in C. elegans (Nigon et al., 1960). The pseudo-membrane started migrating towards the anterior pole before the end of its formation, leaving an anterior lobe of very small size (Figure 14), which only faded after the division. In amphimictic eggs, the meeting of pronuclei occurred in the neighborhood of the posterior pole, when the asters appeared. Spindle oscillations were not observed. 

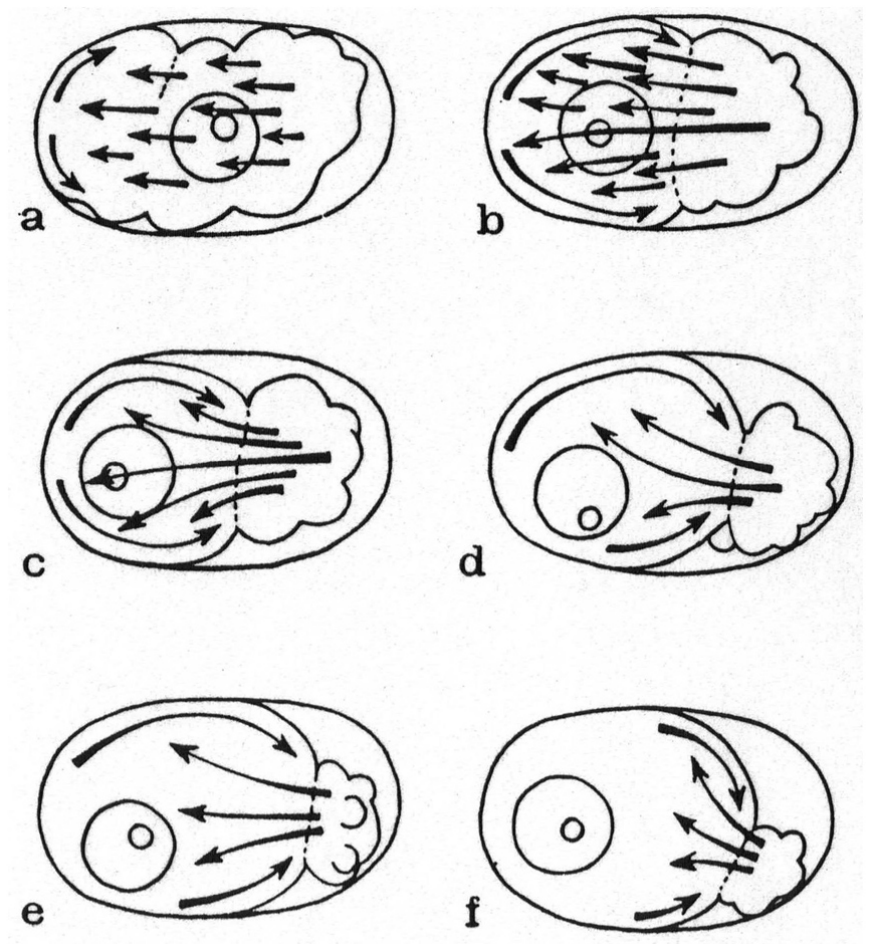

Figure 14. Movements of the plasma membrane (including the pseudocleavage furrow) after fertilization in Mesorhabditis belari. Reproduced from Nigon et al. (1960). Here anterior is to the right.

\subsubsection{Observations on Rhabditis anomala}

In this hermaphroditic pseudogamous species, the single maturation division was observed at the anterior pole, where the polar body remained visible (Nigon et al., 1960). The surface deformations and cytoplasmic streams already started before the maturation division. No pseudomembrane was formed. In a last phase before the division, movements of membrane and cytoplasm were limited to the distal pole before stopping completely. The pronucleus moved slowly towards the center of the egg, before the asters grew. During this movement, the membrane flowed, thereby carrying along the polar body towards the equator. The mitotic spindle appeared in a transverse axis. The spindle then underwent oscillations, elongated and thus aligned along the egg long axis (Figure 12b-d). Exceptionally, the spindle could form when the two asters were already positioned along the egg axis; in this case, the polar body did not move.

Another interesting observation was made on this species and a related species, $R$. maupasi. During spindle elongation, cytoplasmic regions devoid of cytoplasmic granules were formed at both poles, giving the cytoplasm a hyaline appearance. Similar observations had been made in Parascaris by Pasteels (1948), who called these zones polar caps. He noted that at their level pyroninophilic material, likely rich in RNA, was concentrated. These caps could not be seen in C. elegans, perhaps being masked in this species by the high density of granular structures in the cytoplasm.

\section{In today's light}

The first asymmetric cell division of C. elegans as studied in Nigon et al. (1960) has been a model for key discoveries in cell biology, based on forward and reverse genetic tools, visualization, and laser-aided manipulations in this large cell (Cell division; Polarity establishment, asymmetric division and segregation of fate determinants in early C. elegans embryos). The initial reorganization of the cytoskeleton triggered by sperm entry at the posterior cortex and the ensuing actin-network based cortical and cytoplasmic movements during pseudocleavage are now understood in great detail. The role of the cortex in setting up cell polarity, and therefore embryonic polarities, was correctly foreseen by Nigon et al. (1960) and Nigon (1965). The cortical movements result in the differential segregation of molecular determinants of cell fates in the two first blastomeres (Polarity establishment, asymmetric division and segregation of fate determinants in early $C$. elegans embryos). Using mutants defective in egg 
polarization (par mutants), a conserved set of cortical molecules involved in the polarity of many cell types has been discovered. Key conserved molecular players in fundamental cell biological processes such as centriole duplication were also found in C. elegans early embryos. This includes the discovery of the SAS-6 protein that forms the basis for the 9-fold symmetry of centrioles (Cell division; Gönczy, 2012; Hilbert et al., 2013). Also studied are the forces controlling the oscillations of the asters described above (Polarity establishment, asymmetric division and segregation of fate determinants in early C. elegans embryos).

In $C$. elegans and many other rhabditids, the embryonic antero-posterior axis is set by the sperm entry point, even when the sperm entry point is manipulated experimentally (Goldstein and Hird, 1996). By contrast, in Panagrellus redivivus, Acrobeloides nanus, and other nematodes of the Panagrolaimidae and Cephalobidae families, the sperm entry point does not predict the posterior pole (Goldstein et al., 1998). In this case, the direction of the oocyte in the ovary is such that the future anterior pole is towards the vulva, thus the reverse from the usual case in C. elegans (Goldstein et al., 1998). These nematode families contain several truly parthenogenetic groups, where sperm is absent and thus cannot be used for polarity establishment. The family Rhabditidae includes true parthenogenesis in the genus Diploscapter. In this case, the orientation of the embryo axis relative to the orientation of the oocyte in the gonad is random (Lahl et al., 2006); how the axis is established remains unclear. An extensive study of character evolution at different steps in early cell polarity establishment has been performed (Brauchle et al., 2009).

\subsection{Eutely}

The early cell lineage and the adult cell content of specific organs were shown to be invariant in a given species, first when mostly parasitic nematode species were studied (Martini, 1923), and later on the free-living species Anguillula aceti (now Turbatrix aceti, family Panagrolaimidae; Pai, 1928). After a first phase of fast cleavage divisions during embryonic development, cell divisions stop. Then comes a phase when cell types differentiate and organs are formed, without production of new cells. Individuals of the same species have an invariant number of cells in a given organ; this stability in cell number has been called eutely (Martini, 1923).

\section{In today's light}

That the entire somatic cell lineage is mostly invariant has been unequivocally demonstrated by following the entire cell lineage from zygote to adult (Sulston and Horvitz, 1977; Kimble and Hirsh, 1979; Sulston et al., 1983). Development is mostly reproducible in terms of cell division patterns and cell fates. The reproducibility of the cell lineage suggested to Brenner, Sulston, et al. that each cell computed its fate according to its position in the lineage rather than its spatial position in the embryo (Brenner, 2001). Yet this was soon found to be only partially the case since, by the 4-cell stage, cell fates turned out to be specified via signaling between cells. Cell fate specification is now known to occur through a combination of: 1) asymmetric divisions, as that initiated by sperm entry; and 2) intercellular signaling between cells. Reproducible patterns of cell contacts render the cell lineage basically invariant (Schnabel and Priess, 1997; Schnabel, 1997). The mechanisms behind the reproducibility of cell fates are thus relatively well understood, but the regulation of cell cycles in the different sublineages less so.

Although quite invariant in a species, the early cell divisions, the cell lineage, and the partitioning of cell fates (e.g., gut) varies among species (Sternberg and Horvitz, 1981; Sternberg and Horvitz 1982; Sulston et al., 1983; Embryological variation during nematode development; Houthoofd et al., 2008; Schulze and Schierenberg, 2011; Schiffer et al., 2014; Lin et al., 2009).

\section{Post-embryonic development}

Post-embryonic development has been the focus of the first article on nematodes by Maupas, who studied the number of molts and the dauer lifecycle, which he called "enkystment" (Maupas, 1899).

Maupas followed postembryonic development and molts in 13 species (Maupas, 1899). At hatching, all organs were formed, except for the genital apparatus, which was built progressively during post-embryonic development. During each molt, a layer of cuticle was shed and rapidly degraded. Maupas observed four molts in all species, in contrast to previous authors who had reported various numbers of molts.

Maupas also studied the alternative lifecycle through the resistance form (Maupas, 1899). If the animals were deprived of food, for example by letting them hatch inside their mother's body, he observed that "kysts" were formed at the end of the L2 stage, which could persist for several months. In the absence of food, all animals ended 
up dying except for these enkysted larvae. Maupas noticed that only the second molt could give rise to an enkysted larva. The enkysted larva was able to move and had a characteristic behavior, and Maupas inferred that this form was a migration and resistance stage. Some of these enkysted larvae adopted an original transportation mode on other animals; if the host died, they could proliferate on its decomposing corpse. Maupas observed the enkysted dauer form in C. elegans and several other free-living nematodes, but not, for example, in the Cephalobus genus. In this latter genus instead, animals were able to dessicate and "revive" if humidity was restored (Maupas, 1899).

Further variations in post-embryonic development of a given species were noted by Hedwig Hirschmann. She studied dimorphism in mouth morphology in $P$. lheritieri (a gonochoristic species) and a selfing species with rare males, likely P. maupasi (Hirschmann, 1951) (see above, Section 3.5). Hirschmann noted that in a given culture, the adult buccal cavity might adopt either the Stenostoma (or St) form or the Eurystoma (or Eu) form. Larvae all showed a St mouth until the last molt, but in their last larval stage, some individuals underwent a change in mouth morphology to the Eu form, with a wider buccal cavity and additional teeth. The proportion of the Eu form changed with the environmental conditions, increasing in low food and highly crowded conditions. Yet it decreased upon passage through the dauer form (Hirschmann, 1951). In addition, the proportion of the two forms in a given environmental condition varied among different natural isolates of a species. This dimorphism appeared thus to be influenced by the environment and perhaps as well by genetic variation in the species.

\section{In today's light}

The temporal succession of post-embryonic stages and molts are now better understood. Genetic screens for developmental mutants in the Brenner and Horvitz laboratories yielded "heterochronic" mutants, in which the temporal succession of larval stages was altered. These mutants led to the discovery of micro-RNAs, a widespread and conserved mechanism of gene regulation ( $C$. elegans microRNAs). Other aspects of post-embryonic development, such as vulva development (Vulval development), are also now textbook examples in developmental biology.

Free-living nematodes have been a model since the 1980s to study the evolution of development, based first on comparing the pattern of post-embryonic and embryonic cell lineages (Sternberg and Horvitz, 1981, Sternberg and Horvitz, 1982; Sulston et al., 1983; Embryological variation during nematode development; Houthoofd et al., 2008; Schulze and Schierenberg, 2011; Schiffer et al., 2014), then on cell ablation, genetic, and molecular studies (Evolution of development in nematodes related to C. elegans).

The alternative developmental paths-dauer entry vs. direct development-have become a key example of plasticity in animal development that is induced by some environmental parameters. Also, following genetic screens, the alternative choice could be dissected at the molecular and cellular levels (see the WormBook chapter, Dauer). The $C$. elegans dauer larva is particularly relevant to the study of the infective larva of parasitic nematodes (Crook, 2014). Note that Maupas seems to have confused two phenomena: on one hand the dauer larva, which is a third stage larva in its dauer cuticle; on the other hand, the situation where in some rhabditid species the dauer larva remains in a tube formed by the undegraded L2 cuticle. The latter occurs in many rhabditid groups, but not in Caenorhabditis where the L2 cuticle is shed and degraded (Sudhaus, 2011).

Studies of the dimorphism in Pristionchus mouth forms have recently resumed in the Pristionchus genus (Bento et al., 2010; Bose et al., 2012; Bumbarger et al., 2013; Ragsdale et al., 2013).

\section{The alternation of generations}

Some nematode species display both free-living and parasitic generations, often with different reproductive modes. These species likely derive from free-living nematodes. The alternation of generations constitutes in itself an interesting subject: with the same genome, two forms with different development, morphologies, modes of reproduction, behaviors, and lifespans coexist (Nigon and Roman, 1952). When comparing the morphology of free-living and parasitic forms of the same species, large differences could be observed (length varying from 1 to 3 fold or even 1 to 10 fold; much longer lifespan of the parasitic generation; in some cases, extreme modification of the parasitic form). We will here focus on the alternation in their reproductive mode, including gametogenesis and fertilization. 


\subsection{Gonochoristic free-living and hermaphroditic parasitic generations: Rhabdias bufonis}

The first important studies dealing with the chromosomal determination of sex and the alternation of generations were conducted by Boveri (1911) and Schleip (1911) on Rhabditis nigrovenosa (now called Rhabdias bufonis), a nematode species that parasitizes amphibians. The parasitic generation of this species is composed of protandrous hermaphrodites, who give birth to a free-living gonochoristic generation, which in turn produces eggs that develop into infective larvae. Thus, with the alternation of parasitic and free-living forms, the modes of reproduction also alternate, from hermaphroditic to gonochoristic. Given an apparent chromosomal sex determination, two problems arise: how can a hermaphrodite give rise to both females and males, and conversely how can females and males give rise to hermaphroditic "females" only?

The parasitic hermaphrodites of Rhabdias bufonis display a diploid number of 12 chromosomes in their mitotic germ line. After two normal maturation divisions, the oocyte contains a standard haploid content of 6 chromosomes. Spermatogenesis in hermaphrodites is more complex, producing spermatozoa with 6 or 5 chromosomes. How can a hermaphrodite with 12 chromosomes give rise to these two kinds of sperm? According to Boveri, spermatogenesis may entail the loss of one chromosome (as in O. dolichura, see Section 3 and Section 4.2). In contrast, according to Schleip, the maturation of spermatocytes proceeds normally, giving rise to spermatids with 6 chromosomes, and it is only during the transformation of spermatids into spermatozoa that one chromosome would be degraded in some spermatids, producing spermatozoa with 5 chromosomes. In any case, fusion of these gametes gives rise to the free-living generation: those with 12 chromosomes are females, while those with 11 are males.

Concerning the second question, the authors stated that in the free-living generation, gamete formation occurred normally, with two maturation divisions. They thus gave rise to oocytes with 6 chromosomes in the females and sperm with 5 or 6 chromosomes in the male. The development of hermaphroditic "females" only in the next generation (parasitic) would derive from the fact that spermatozoa bearing 5 chromosomes were inactive. This last conclusion was hypothetical.

\subsection{Gonochoristic free-living and parthenogenetic parasitic generations: Strongyloides ratti}

Studies on species with alternating generations continued, but few of them dealt with the determination of the succession of generations and their reproductive mode. Nigon and Roman (1952) collaborated on a first work in this field on Strongyloides ratti, a rat parasite. Roman maintained one line of Strongyloides ratti for several years in the laboratory, while Nigon performed cytological investigations. In $S$. ratti, the parasitic generation is parthenogenetic, while the free-living generation is gonochoristic (Figure 15).

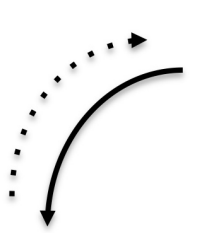

parasitic adults females $(X X)$

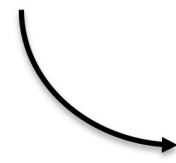

filariform

infectious

larvae

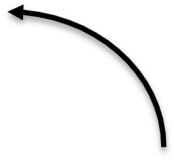

free-living adults

females $(X X)+$ males $(X 0)$

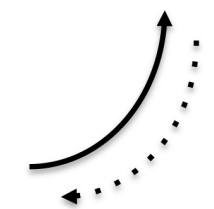

Figure 15. Schematic lifecycle and sex determination in Strongyloides ratti. After Nigon and Roman (1952). The dotted lines indicate that successive parasitic or free-living generations are possible.

In parasitic females, the mitotic zone of the ovary shows a diploid stock of 6 chromosomes, which do not pair in early meiosis. The oocytes develop in a parthenogenetic mode without fertilization, with a single maturation division. Parasitic parthenogenetic females give birth to rhabditiform larvae that escape through the host feces (stercoral generation). 
Nigon and Roman (1952) found that the development of the next generation depended on external factors:

1). If the larvae were cultured on an agar medium, only males reached the adult stage. They represented approximately $0.5 \%$ of the population. The other larvae developed into filariform infective larvae, which might infect rats when applied to their skin. Some of them would reach the intestine and further develop into parasitic females.

2). If the larvae were raised in coproculture (on feces), the production of males was not modified, but adult females were also formed in a number similar to that of males. This free-living generation was composed of two sexes. The remainder (i.e., $99 \%$ of the progeny of the parasite) developed into infective larvae.

In the free-living generation, females displayed a diploid stock of 6 chromosomes, and the males only had 5. Non-disjunction of the sex chromosome in the parasitic female parent was hypothesized but not actually observed, which was not surprising given that non-disjunction should only take place in $0.5 \%$ of the meioses.

In the oocytes of free-living females, Nigon and Roman (1952) observed 3 bivalents at diakinesis. The oocytes were fertilized by sperm from the free-living males, and a fertilization membrane was formed. The oocyte nucleus migrated to the plasma membrane, apparently preparing for its first reductional division, with homolog pairing. But at metaphase, the bivalents separated and formed an equatorial plate with 6 chromosomes. The unique maturation division splitted the 6 chromosomes, and a polar body was extruded. Thus the maturation division started like a reductional division but ended in an equational division. During this time, the sperm was condensed into a small DNA mass that could later be found in one cell of the cleaved embryo. This behavior thus corresponded to a pseudogamous parthenogenetic reproduction, as described above for M. belari (Section 5.2.3). The absence of true fertilization would explain why only females were formed in the next infective generation.

Some observations of Nigon and Roman suggested the presence of rare alternative behaviors. In one egg, they described the presence of two pronuclei, each containing 3 chromosomes; no condensed sperm was found in this egg. This configuration thus corresponded to a normal fertilization, with two haploid pronuclei. In the same animal, another egg displayed the most usual pseudogamous behavior. Thus, as in M. belari, in the same individual two modes of meiosis and fertilization could co-exist. Some eggs also appeared to contain polyploid nuclei, with up to 24 chromosomes. In these eggs, the sperm remains were identifiable, but no polar bodies were seen, suggesting the occurrence of abnormal maturation divisions. Thus, next to the known parthenogenetic development, variant behaviors could be observed in free-living females, with unclear consequences.

In free-living males, spermatogenesis occurred with many irregularities. A detailed study was not undertaken. Observations of the development of the eggs showed that in most cases, the male genome did not participate to reproduction. However, in some cases, at an unknown frequency, amphimixis could be observed, which sugested the existence of a spermatozoon with 3 chromosomes.

In conclusion, the free-living gonochoristic generation from the strain under study in uniform culture conditions reproduced mostly via pseudogamous parthenogenetic reproduction, more rarely through amphimictic reproduction, as in $M$. belari. The development of amphimictic eggs has not been studied.

\section{In today's light}

More recently, alternative lifecycles of Rhabdias bufonis have been studied by Spieler and Schierenberg (1995). They showed that while at hatching, larvae of both free-living and parasitic generations display a similar number of nuclei, the parasitic adult (of larger size) contains many more nuclei than the free-living generation. This is in part due to the presence of over 2000 nuclei in the intestine, versus about 20 in the free-living generation as in C. elegans.

Concerning Strongyloides ratti, excellent recent reviews are those of Streit (2008) and Viney and Lok (The biology of Strongyloides spp). Viney studied many strains of $S$. ratti, with a well-controlled culture technique, and found that coproculture gave rise to a high proportion of free-living females. Development in free-living adults versus infective larvae was governed by many factors including the strain, the culture conditions and the immunological status of the host rat (Viney et al., 1992; Viney, 1996; Harvey et al., 2000). 
The reproduction mode of the free-living generation of $S$. ratti remains controversial and it is possible that the character varies among closely related species (Streit, 2008). Through studies of the segregation of molecular genetic markers, Viney et al. confirmed that the parasitic generation of $S$. ratti reproduced parthenogenetically (Viney, 1994) and that males carried a single copy of one chromosome, consistent with XX/X0 sex determination (Harvey and Viney, 2001). However, in contrast to the cytological studies by Nigon and Roman (1952), they found, using genetic markers, that the free-living adults reproduced through true fertilization (amphimixis) (Viney et al., 1993; Nemetschke et al., 2010b). The discrepancy between the cytological studies and the later genetic studies remains unexplained (Streit, 2008). The occurrence of pseudogamy versus amphimixis, or their relative frequency if they coexist, may depend on external factors as well as on the genetic composition of different $S$. ratti lines (or cryptic Strongyloides species). Studying the cytology of meiosis and fertilization on lines where amphimixis seems prevalent appears useful at this point. Ongoing research indicates an exciting future for studies on this species and closely related species (Nemetschke et al., 2010a).

A new convenient model system to study the alternation of generations with different modes of reproduction is now available, without an obligate parasitic stage. The species provisionally called Rhabditis sp. SB347 develops on agar plates seeded with $E$. coli, either through a generation that adopts the dauer stage and develops into hermaphroditic adults, or through a gonochoristic generation that does not pass through the dauer stage (Félix, 2004). The ecology of the species is unknown but it is possible that the dauer stage corresponds to passage in a host. Morph and sex determination in this species are being studied in the laboratory of Andre Pires daSilva (Chaudhuri et al., 2011). The paucity of males in the progeny of the gonochoristic generation has been explained by the asymmetry of spermatocyte division and the selection of the X-bearing sperm spermatocyte, vindicating the hypothesis of Boveri and Schleip for R. bufonis (Shakes et al., 2011).

\section{Genetics}

\subsection{Preliminaries}

Dougherty and Hermione G. Calhoun were the first to suggest the potential relevance of species of the rhabditids for studies in formal genetics (Dougherty and Calhoun, 1948a). Dougherty foresaw the advantages that the reproductive modes (Dougherty, 1949) and the developmental speed could bring to genetic studies. The selfing mode of reproduction appeared particularly convenient to produce homozygous mutant animals. Dougherty hoped that the possibility of culture on defined media could lead to studies in physiological genetics inspired by those of Beadle and Tatum on Neurospora.

\subsection{The first mutants}

While the first genetic variant in C. elegans was the tetraploid line (Nigon, 1949b; Nigon, 1951a) (see Section 4.4), the first description and analysis of a recessive mutation was in C. briggsae (Nigon and Dougherty, 1950). This mutation appeared in a culture that had been exposed to a temperature higher than $23^{\circ} \mathrm{C}$. In the adult stage, this mutant was approximately a quarter shorter than the wild-type, hence its designation as micro. Its width was identical to that of the wild type, giving it a stocky appearance (Figure 16). Its fecundity and motility were reduced. 


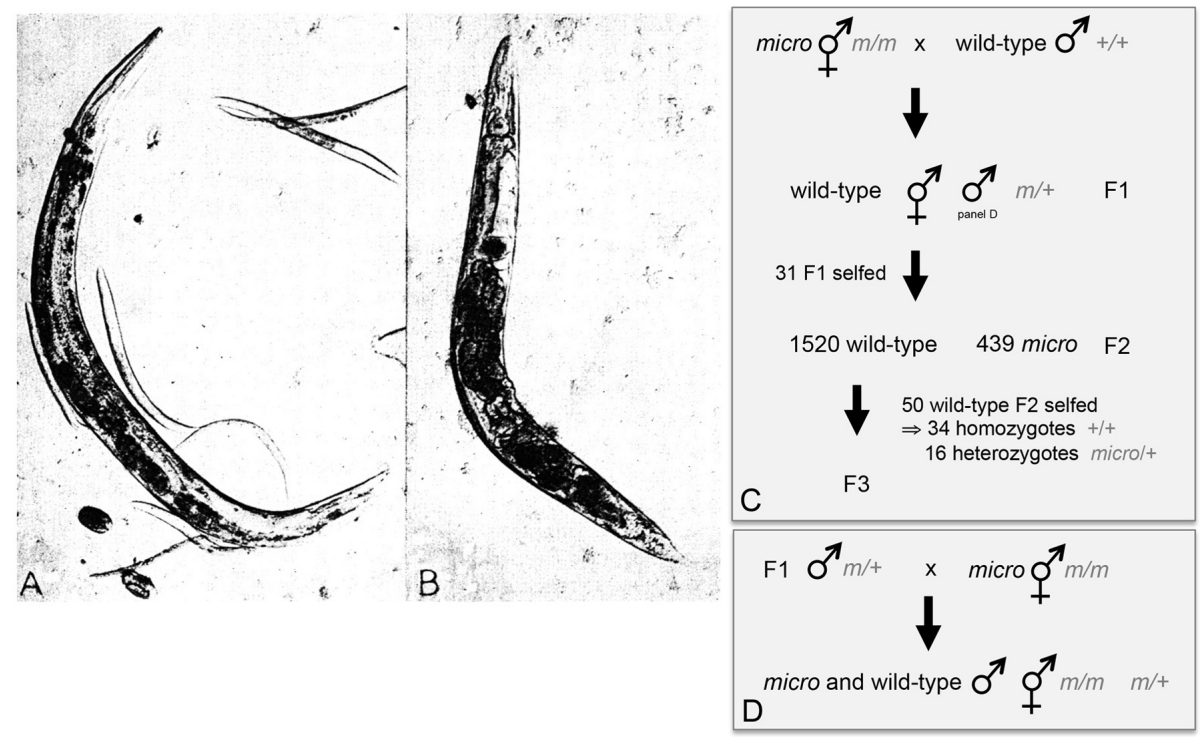

Figure 16. The C. briggsae micro mutant. From Nigon and Dougherty (1950), with permission from Oxford University Press. (A) Wild-type C. briggsae hermaphrodite adult. (B) micro mutant. (C) Cross of the micro mutant with wild-type males and segregation by selfing of hermaphrodites in the F2 and F3 generations. Genotypes are in grey. The micro mutation segregates as a single locus. (D) Backcross of the F1 males to mutant hermaphrodites, showing absence of linkage to the sex chromosome.

Crosses of micro hermaphrodites with wild-type males gave rise to heterozygote progeny of wild-type phenotype, both males and hermaphrodites. When the F1 hermaphrodites were selfed, the F2 generation showed a segregation of wild-type and mutant phenotypes. The F3 progeny from F2 individuals of wild-type phenotype were further followed (Figure 16C). The values for the F2 genotypes were close to the 1:2:1 ratio expected for the F2 progeny of a F1 hybrid between wild-type and homozygous recessive mutant parents. In addition, F1 males backcrossed to micro hermaphrodites yielded hermaphrodites and males, both of wild-type and mutant phenotypes (Figure 16D). The F1 males were thus heterozygous, showing that the mutation is not sex-linked. It was concluded that the micro character depended on an autosomal recessive mutation.

Several attempts were made by Dougherty and Nigon to study the effect of chemical mutagens. The first experiments, performed in Paris in 1949, made use of yperite and acriflavine (Nigon and Dougherty, 1953; Dougherty and Nigon, 1953). C. elegans did not provide a variety of obvious mutant phenotypes. Treatment with yperite resulted in a $C$. elegans strain with variable phenotypes, named "pygmy", which differed from the micro mutants (Nigon and Dougherty, 1953). These animals grew with a variable rate, ranging from normal speed to very slow growth. For those reaching the adult stage, their shape and size were close to those of normal individuals of this species, and some died before reaching the adult stage. This form was maintained for hundreds of generations by selfing, without modification of the phenotypic variability. Nigon pursued studies on the variable expression of the phenotype, trying to select phenotypic extremes in the pure line (Nigon, 1954).

\section{In today's light}

The spontaneous micro mutant in C. briggsae corresponds to a common class of mutants found in C. elegans and other rhabditids, now called dumpy (abbreviated dpy) following Brenner (1974). The slow-growing pygmy mutant with variable expression (Nigon and Dougherty, 1953) was likely what is now called gro (abnormal GROwth rate) or $c l k$ (CLocK, biological timing abnormality) mutants, which display reduced growth rates. Nigon was interested by the variability of its phenotype, which may have reflected the variable expressivity of such mutants.

Together with the tetraploids, these mutants marked the beginning of $C$. elegans and $C$. briggsae genetics. Reasons why Nigon and Dougherty did not carry on with mutageneses are discussed in Section 12.1.1.

\subsection{Natural variation in temperature resistance}

The sensitivity of C. elegans (and other species) to high temperature was noted by Maupas (1900). In Bergerac, Nigon collected C. elegans twice (Appendix 2, Section 17). The first strain, sampled in 1941, could 
multiply well at $25{ }^{\circ} \mathrm{C}$. The second strain (known today as Bergerac) isolated in March 1944, displayed abnormalities already at the temperatures of $23-24{ }^{\circ} \mathrm{C}$ (Nigon, 1949a). Only this second strain was studied by morphologists (Dougherty, Osche, etc.) and confirmed by them as $C$. elegans. It is possible that the first isolate might have been another species, such as $C$. briggsae, or a $C$. elegans with normal resistance to temperatures of $23-25^{\circ} \mathrm{C}$.

The C. elegans strain from Bristol is indeed still fertile at $25^{\circ} \mathrm{C}$. The transmission of the genetic difference in heat tolerance between the 1944 Bergerac and the Bristol strains of $C$. elegans was studied by Helene Fatt and E. Dougherty (Fatt and Dougherty, 1963). At that time, crosses between the Bergerac and Bristol strains could not be made in the axenic liquid media used in the Dougherty lab, so they used agar plates seeded with E. coli for the crosses and, after antibiotic treatment, brought them back to axenic liquid culture. The heat tolerance of Bristol was found to be dominant and to segregate in an approximate 3:1 ratio. Analysis of individual heat-tolerant F2s yielded a ratio of 1 homozygous to 1.5 heterozygous, not too distant from the expected 1:2. Following a first cross of Bergerac hermaphrodites with Bristol males and a backcross of F1 males to Bergerac, some F2 progeny were heat-tolerant-thus the transmission was not X-linked but autosomal. In conclusion, heat sensitivity of Bergerac vs. Bristol was transmitted as a recessive autosomal trait. This is the first example of genetic analysis of a natural variation in C. elegans.

This genetic study was followed by an attempt by Fatt to characterize factors of the culture medium that participated in inhibiting progeny production of the Bergerac strain at high temperature. These environmental manipulations were performed by changing the composition of the axenic medium. Fatt found that at high temperature Bergerac individuals produced an inhibitory substance, whereas Bristol did not (Fatt, 1967).

\section{In today's light}

The major autosomal locus responsible for the difference in heat tolerance between the Bergerac and Bristol strains of C. elegans has been mapped to chromosome II and the locus called zyg-12 by Wood et al. (1980); it was mapped again by Shook et al. (1996). The gene and molecular lesion were identified by Malone et al. (2003). The Bergerac allele is a thermosensitive reduction-of-function missense mutation (ct350, an A to C change) in the zyg-12 gene. This gene encodes a transmembrane protein of the conserved Hook family that links the centrosome to the nucleus (Sato et al., 2009), and through the nuclear envelope also participates in chromosome pairing in early meiosis as well as in the overall architecture of this part of the gonad (Penkner et al., 2007; Penkner et al., 2009; Zhou et al., 2009). The temperature-sensitivity of the Bergerac allele appears to originate in the binding inability of the Bergerac ZYG-12 protein at high temperature (Malone et al., 2003).

The presence of this temperature-sensitive zyg-12(ct350) allele in the C. elegans Bergerac explains several temperature-dependent phenotypes that were observed by Nigon and colleagues, such as unpaired chromosomes in early meiosis and polyploid nuclei in the gonad (Nigon, 1951b; Brun, 1955), centrosomes detaching from the male pronucleus after fertilization, and the two pronuclei staying apart resulting in embryonic cleavage defects (Nigon et al., 1960). Note that independent of the zyg-12 mutation, chromosome pairing in meiosis is also temperature-sensitive in the Bristol/N2 background, which may explain the increased male production at high temperature (Bilgir et al., 2013). Thus, although the specific zyg-12 mutation makes some cellular reactions particularly sensitive to high temperature for Bergerac animals, other processes are similarly temperature-sensitive in the different wild strains of $C$. elegans.

\subsection{Classical genetics in Brun's team before 1974}

Jean-Louis Brun was a student of Nigon in Lyon (see Appendix 3, Section 18.6). Brun started classical genetic experiments with $C$. elegans, after performing the high temperature experiments described in Section 10, at a time when Nigon himself had left the worm and gave Brun the chance to conduct his own research. With Michel Dion, Brun first isolated and analyzed two spontaneous dwarf (dumpy) mutations in the C. elegans Bergerac background (Dion and Brun, 1971). These mutations were found to be recessive, autosomal and unlinked. One of them is available at the CGC as FF1, of genotype $d p y-5(f 1)$ (M. Whistler, D. Riddle, P. Swanson, personal communication, The Worm Breeder's Gazette, 7 (1): 50). Cadet and Dion, informed by Brenner (personal communication in Dion and Brun, 1971), then reported on the use of ethyl-methane sulfonate (EMS) as a mutagen. After mutagenesis they isolated seven other dwarf mutants, as well as one mutant with a defect in locomotion (Cadet and Dion, 1973). X-ray mutagenesis was also used (Ouazana and Brun, 1975). 
Complementation and linkage studies were performed (Dion and Brun, 1971) (Figure 17). Using five alleles of what is likely $d p y-1$ III (Ouazana, 1975), intragenic recombination could be detected and measured (Ouazana and Brun, 1975).

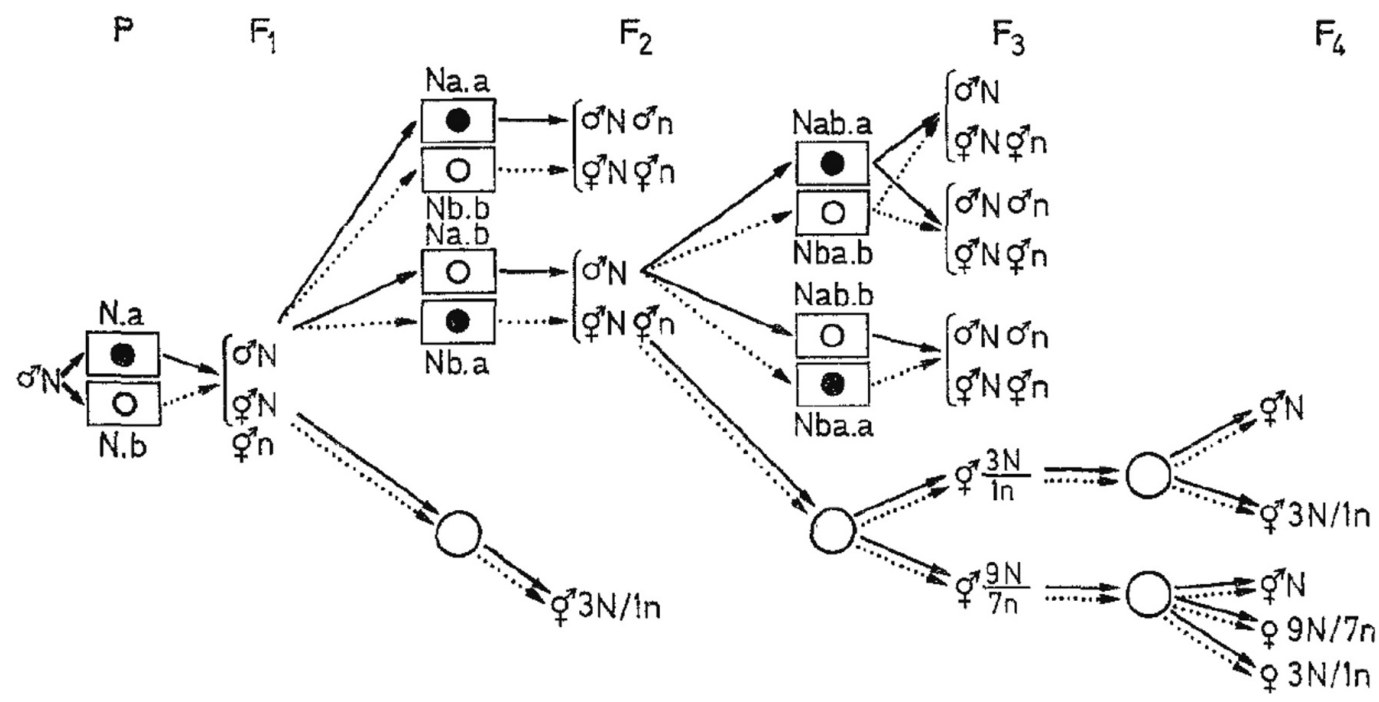

Figure 17. Diagram of crosses testing for the monofactorial segregation of recessive mutations, and allelism and genetic linkage of two dwarf mutations. From Dion and Brun (1971), with permission from Springer Publishing Company. Rectangles: crosses leading to the next generation. White circle: self-fertilization. N: normal phenotype. n: dwarf phenotype ('nain' in French). The proportion of 3 normal animals to 1 dwarf in the F2 generation (bottom center) indicated that the mutation was recessive and monofactorial. 'N.a' indicates a cross of a normal male with a n hermaphrodite, 'Na.b' of the hybrid F1 male to a $n_{b}$ hermaphrodite, etc. The latter cross showed that the dwarf mutations a and $b$ were not allelic. On the lower right, the proportion of 9 normal to $7 \mathrm{dwarfs}$ corresponded to the case of two unlinked mutations; a proportion of $3 \mathrm{~N}$ to $1 \mathrm{n}$ corresponded to closely linked mutations. Black and white dots, as well as full and dotted arrows, corresponded to mutations a and b, respectively.

\subsection{Classical genetics in Brun's team after 1974}

We will be brief on this part that came after the first publication on C. elegans by Brenner in 1974. After this, the Brun team published on a variety of mutant phenotypes, such as a high incidence of males (called Him) (Béguet, 1978), a transformation of the hermaphrodite tail towards a male tail (Béguet and Gibert, 1978) (identified as a weak tra-2 allele by Hodgkin (1985)), temperature-sensitive lethality and sterility (Abdulkader and Brun, 1976; Abdulkader and Brun, 1978, 1980; Abdulkader et al., 1980), as well as unconditional sterility (Mounier and Brun, 1980; Gibert et al., 1984). Brun passed away in 1981, but the team continued until the end of ongoing PhD theses. Overall, one focus was the dumpy mutants (Ouazana and Brun, 1978; Ouazana, 1978) and collagen structure (Ouazana and Gibert, 1979; Ouazana and Herbage, 1981; Ouazana et al., 1985), because many dumpy mutations affect collagen genes. The second focus was gametogenesis and the sterile mutants, because of the central importance of gametogenesis in reproduction (Abi-Rached and Brun, 1975; Abirached and Brun, 1978; Abirached and Brun, 1979; Starck et al., 1983; Starck, 1984). The sterile mutants were, however, more difficult to maintain and study than the classes first chosen in the Brenner lab, such as uncoordinated mutants or cell lineage mutants with egg-laying defects.

Other than the tra-2 allele that was crossed into the N2 background (Hodgkin, 1985), these later mutants, all in the Bergerac background, do not seem to have been preserved.

\section{In today's light}

Genetic complementation and linkage were developed in the team of Brun. Although Dougherty and Brun agreed that they needed to develop genetics, their attempts were dwarfed by the extent (number of mutants and genetic map), the phenotypic diversity, and the biological relevance of the first mutageneses performed by Brenner et al. The Brun team later started isolating a variety of mutant phenotypes, possibly following contact with the budding C. elegans community (particularly David Hirsh). A strength of Brenner's work was to find many locomotion-defective ("uncoordinated") mutants that allowed him, crossing them with the dumpy mutants, to build a genetic map (Brenner, 1974). Using dwarf mutants alone, linkage analysis was more arduous (Dion and Brun, 1971). This is an example of the awkwardness in the use of genetics by the French team, which was isolated in Lyon from geneticists in France and elsewhere. 
Another problematic issue was the divergent use of the Bergerac and Bristol strains. While the rest of the world adopted Bristol, which developed better at temperatures above $20^{\circ} \mathrm{C}$, the team in Lyon had naturally carried on with mutagenesis of the Bergerac strain. This may have contributed to their mutants having been little used and forgotten.

\section{Adaptation to high temperature}

In 1966, Jean-Louis Brun published a series of three papers reporting on the dynamics and reversibility of laboratory adaptation of $C$. elegans Bergerac to a higher temperature (Brun, 1966a, b, c). These papers corresponded to his thesis under the supervision of Nigon in Lyon. The adaptation experiment was also briefly summarized in English in Science (Brun, 1965). The results of these papers remained mostly unknown, yet their significance may be re-evaluated in the light of recent results on epigenetic inheritance in C. elegans.

\subsection{Progressive sterility and acclimatization behaviors}

In an experiment running over thousand generations and several years, Brun progressively adapted the Bergerac line to higher temperatures, using the following protocol. At each generation, 6-10 young adult progeny from the same parent were isolated (each in a separate culture), in the high temperature lines (cultured at temperatures ranging from $22-24.5^{\circ} \mathrm{C}$ ) and in an $18{ }^{\circ} \mathrm{C}$ control line. Selection was applied in the sense that: 1) the chosen brood was that with the most abundant progeny at this (early) time, and without aberrant-looking individuals; and 2) the individuals were chosen among the first descendants, so as to avoid crossing with males.

Brun first defined four types of behavior of the Bergerac line when brought to a range of high temperatures (Brun, 1966a) (Figure 18). The Bergerac line was routinely kept at $18{ }^{\circ} \mathrm{C}$, without signs of sterility. Above $24{ }^{\circ} \mathrm{C}$, the strain was immediately sterile. At 23 or $23.5^{\circ} \mathrm{C}$, the strain only became sterile after several generations, with a progressive decrease in brood size. This multigenerational phenotype resembles that now called Mortal germ line (Mrt) (Ahmed and Hodgkin, 2000). At $22^{\circ} \mathrm{C}$, the strain remained fertile, and after 100 generations, was able to sustain $23{ }^{\circ} \mathrm{C}$ without a Mrt phenotype: the line had thus adapted to a higher temperature regime.

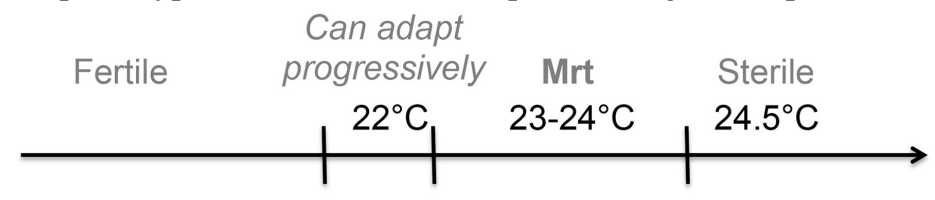

Figure 18. Fertility properties of $\boldsymbol{C}$. elegans Bergerac at different temperatures. Redrawn from Nigon and Brun, (1967). 'Mrt': sterility arising after several generations, now called the 'Mortal Germ Line' phenotype.

Brun then continued using this method of acclimatization, from $22{ }^{\circ} \mathrm{C}$ to $23{ }^{\circ} \mathrm{C}$, then using $0.5{ }^{\circ} \mathrm{C}$ steps of 100-450 generations each, until, after over 1000 generations, the culture could be maintained at $24.5{ }^{\circ} \mathrm{C}$, a temperature at which it was initially sterile. This acclimatization was reproducible when parallel lines were followed, with a similar timecourse: a first rapid decrease in brood size when the strain was shifted to the higher temperature (over 2-10 generations-potentially leading to a Mrt phenotype), a phase of rapid recovery (over $\sim 100$ generations), followed by a late phase with a slower increase in brood size (up to 500 generations). During this last phase, the line became finally able to withstand the next $0.5^{\circ} \mathrm{C}$ increase.

\subsection{Reversibility of the adaptation and effect on the germ line}

When brought back to $18{ }^{\circ} \mathrm{C}$, high-temperature adapted lines showed a higher brood size than the control 18 ${ }^{\circ} \mathrm{C}$ line for several generations. The effect was cumulative: brood size upon return to $18{ }^{\circ} \mathrm{C}$ increased as a function of the adaptation temperature and the duration of culture at this temperature. This effect of long exposure to high temperature was reversible and brood size then gradually decreased over $10-100$ generations of return to $18{ }^{\circ} \mathrm{C}$.

Upshifting the temperature again after a few generations at $18{ }^{\circ} \mathrm{C}$, Brun further showed that the adaptation to high temperature was rapidly lost after as few as 7-10 generations at $18{ }^{\circ} \mathrm{C}$ (Figure 19). He repeated these observations with several lines and several adaptation and reversion temperatures in a reproducible and apparently progressive fashion (Brun, 1966b). Thus, adaptation to high temperatures was slow-over hundreds of generations-but reversible over a few generations of selfing at $18{ }^{\circ} \mathrm{C}$. 
High temperature
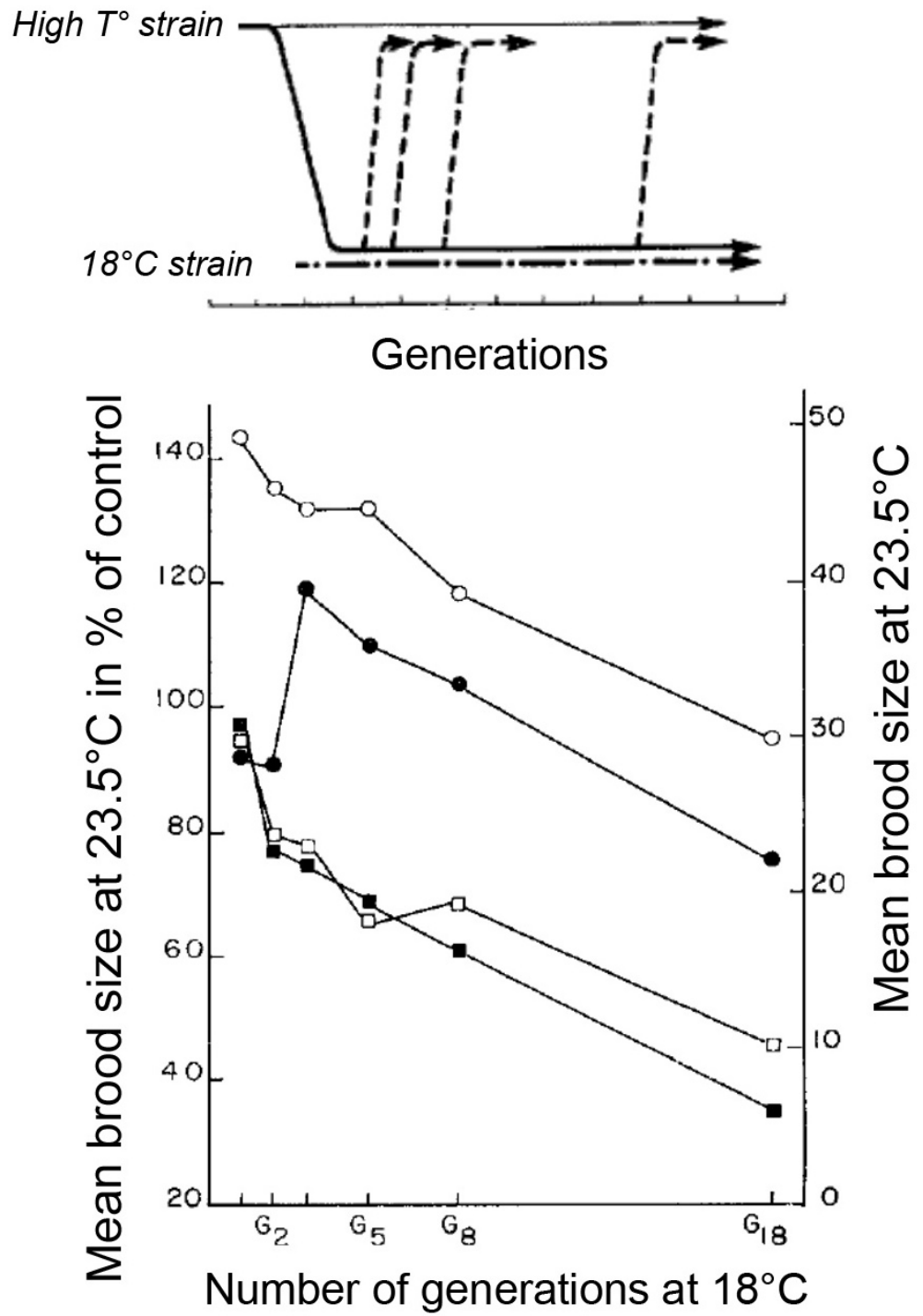

Figure 19. Progressive decline in brood size at high temperature of down-shifted cultures as a function of the number of generations spent at $\mathbf{1 8}^{\circ} \mathrm{C}$. Top: design of the experiment, with temperature in the vertical axis, generations in the horizontal axis. The high-temperature adapted strain was down-shifted to $18^{\circ} \mathrm{C}$ for a varying number of generations and up-shifted again at $23.5^{\circ} \mathrm{C}$. A control was maintained at $18^{\circ} \mathrm{C}$. Bottom: brood size after return to high temperature, as a function of the number of generations at $18^{\circ} \mathrm{C}$ (horizontal axis). Filled symbols: mean brood size in $\%$ of the $18^{\circ} \mathrm{C}$ control (left scale). Empty symbols: mean brood size in absolute value (right scale). Circles: highly adapted line ' 453 ', squares; less adapted line '277'. Top panel adapted from Nigon and Brun (1967), with permission from Springer for Verlag Birkhauser. Bottom panel adapted from Brun (1966b).

Cytologically, high-temperature shift of the control line caused defects in oogenesis in young adults, such as a lack of oocytes in the synapsis and pachytene zone, and the presence of oocytes with multiple nuclei or giant polyploid nuclei in the diakinesis zone. These phenotypes were absent in the adapted line.

\subsection{Hypotheses of non-genic factors and induction by high temperature}

The final paper of the series (Brun, 1966c) discusses possible factors underlying the observed temperature adaptation. First, evolution in the bacterial community used as food and uncontrolled variation in agar medium composition were ruled out by control experiments. Second, the presence of sufficient initial genetic variation that could be selected upon was also ruled out by the selfing nature of $C$. elegans and the maintenance regime with a single individual prior to the experiment. Third, de novo mutations surely occurred over hundreds of generations and could have been selected for; some temperature steps may possibly have been achieved by such incoming genetic variation. Yet Brun disfavored the role of genetic evolution by mutation in his experiment for several reasons: 1) the 
overall continuous adaptation over the five temperature steps, 2) the reproducibility of the adaptation schedule in different lines and at different temperatures, and 3) the rapidity and reproducibility of the reversion in adaptation upon return at a lower temperature.

Having ruled out that the above factors were sufficient to explain the adaptation dynamics in his selfing lines, Brun proposed that the observed heritable adaptation to high temperature occurred through non-genetic changes ("genetic" or "genic" is here used to mean based on DNA sequence, whereas "heritable" refers to transmission between generations, which is not necessarily DNA-sequence based, e.g., through small RNAs and histone modifications). Brun envisioned on one hand heritable non-genic sterilizing processes (called S) that would decrease fertility across generations, and on the other hand a slower acclimatization process (called A) that would slow down the fertility decline. He hypothesized that the acclimatization could work either through variation and selection on the heritable sterilizing processes and/or of a physiological regulation induced by the environment that would inhibit these processes or their heritability. If the modifications were originally induced by the environment, they then became heritable and partially independent of it. The Science article ended with: "Comprehensive observations support the assumption that adaptive transmissible cytoplasmic states are produced gradually and are responsible for the production of fertile high-temperature strains" (Brun, 1965).

The Brun 1966 articles were further summarized in Nigon and Brun (1967), yet hardly cited outside of Brun's group. They inserted themselves well in the contemporary scientific context of some adaptive heredity literature (Lansing, 1954; Jinks, 1964), and were indeed cited on this behalf (Davitashvili, 1969) and more recently (Pal and Miklos, 1999; Tal et al., 2010). Yet they remained unexplained in terms of possible mechanisms and did not fit well with the increased use of standard genetics in both laboratory and evolutionary biology at that time.

\section{In today's light}

Almost fifty years later, the progressive Mortal germ line (Mrt) phenotype observed by Brun in the Bergerac strain needs yet to be reproduced and explained. This phenotype has not been detected in C. elegans N2, but has been observed in many temperature-sensitive mutants derived from it, affecting : 1) telomere formation and DNA repair (Smelick and Ahmed, 2005; Kirienko et al., 2010), or 2) histone modifications and small RNA synthesis in the germ line (Buckley et al., 2012; Simon et al., 2014). Because germ line small RNAs and histone modifications are transmissible across generations in C. elegans (Grishok et al., 2000; Burton et al., 2011; Gu et al., 2012; Buckley et al., 2012; Ashe et al., 2012; Shirayama et al., 2012; Conine et al., 2013), they could perhaps explain a non-genic transgenerational effect or adaptation.

A feature that is now known to differ between the Bristol (N2) and at least some Bergerac-derived strains is the level of germ line transposition-much higher in the latter-although it greatly varies among derivatives of the Bergerac strain (Emmons et al., 1983; Liao et al., 1983; Moerman and Waterston, 1984) (see Appendix 2, Section 17). Interestingly, some of the mutants with a Mrt phenotype in the $\mathrm{N} 2$ background also have an increased transposition rate, through the lack of matching small RNAs (Das et al., 2008; Bagijn et al., 2012). It is so far unclear whether the high level of transposition in both Bergerac and these mutants is a/the process leading to sterility after several generations. Other processes, such as progressive deregulation in gene expression, have also been proposed in the case of germ line small RNA pathway mutants (Buckley et al., 2012).

A few years after Brun's experiments, Hansen, Lower, Yarwood et al,, past collaborators of Dougherty (Lower, 1966), adapted large populations of C. briggsae and P. redivivus (Lower et al., 1968; Lower et al., 1970) to high temperatures, with an interesting axenic culture design resembling Drosophila population cages (Hansen and Cryan, 1966). In this work, adaptation was presumed to be genetic and Brun's work was not cited. The authors did not test for the stability of the adaptation.

\section{Effect of parental aging on fertility of the next generation}

Brun later studied with Danielle Lebre and Bernard Béguet the effect of parental age on the brood size of the first and subsequent generations. Using the Bergerac strain, they uncovered an effect of mother's age on the F1 brood size at $18{ }^{\circ} \mathrm{C}$, through both a decrease in sperm number and an increase in embryonic lethality (Brun and Lebre, 1968; Béguet and Brun, 1972). In a design similar to that of Lansing (1954) in a rotifer, Béguet then continued with what he called the Y (Young) and O (Old) series, derived at each generation from young or older mothers, respectively. The effect of age was reversible in one generation after return to the Y series (Béguet, 1972). The $\mathrm{O}$ series gradually adapted to its treatment by returning to a brood size equivalent to both the starting level and the parallel Y series, within five generations. 


\section{In today's light}

It is possible that the effect of age is related mechanistically to the multigenerational Mrt phenotype. At the least, it suggests that Brun's choice to pick the first progeny during his temperature adaptation may have driven some adaptive phenotypic effect.

Experiments on the effect of aging on next-generation brood size have not been performed more recently to our knowledge. More generally, little attention has been paid to the effect of parental age. Nigon (1949a) had first shown in $M$. belari an effect of parental age on the proportion of eggs developing through pseudogamy versus true fertilization. A lack of parental effect was observed on the longevity of C. elegans progeny (in contrast to rotifers and other animals) (Klass, 1977). In recent studies with C. elegans, the effect of parental age has been paid little attention to, or only to control for it and prevent its interference. Parental age was however observed to decrease recombination frequency at meiosis (Rose and Baillie, 1979; Lim et al., 2008; Goldstein and Curis, 1987). More recently, oocyte quality was shown to decrease with age through regulation by the TGF $\beta$ and insulin pathways (Luo et al., 2010). Parental age also has a dramatic effect on progeny survival in a cross between $C$. elegans wild isolates displaying incompatibilities at the zeel-1/peel-1 locus, with the older progeny surviving better. This effect was hypothesized to depend on sperm size (Seidel et al., 2011). The decreased (or increased in the latter case) quality of late progeny is likely to have important consequences on population dynamics.

\section{Before and after Sydney Brenner}

Historical accounts of the development of $C$. elegans as a model organism usually neglect early studies that used C. elegans (Figure 20) and other free-living nematodes as model organisms to address general questions in biology; they start with Brenner (de Chadarevian, 1998), or only cite Maupas for the species description and Dougherty for sending the Bristol strain to Brenner (Ankeny, 2001; Brown, 2003). Brenner undoubtedly had a pivotal importance in the history of $C$. elegans, made it a key model organism, and achieved much with it that his predecessors had failed to develop. In his 1974 paper (Brenner, 1974), he cited studies by Dougherty, Nigon, and Brun (Figure 21); the first and second "Wormbook" (Wood, 1988; Riddle et al., 1997) also cited many early publications. In later historical accounts however, the earlier work became neglected (see Hodgkin, 2002b for an exception). This neglect may be explained by several factors, including the language of publications (French mostly, and German for some other species), the fact that Nigon and Dougherty were no longer in the field when Brenner started, the focus on the brilliant figure of Brenner, the shift to a new reference strain, ... and the mere passage of time and simplification of the historical narrative. 
History of research on C. elegans and other free-living nematodes as model organisms

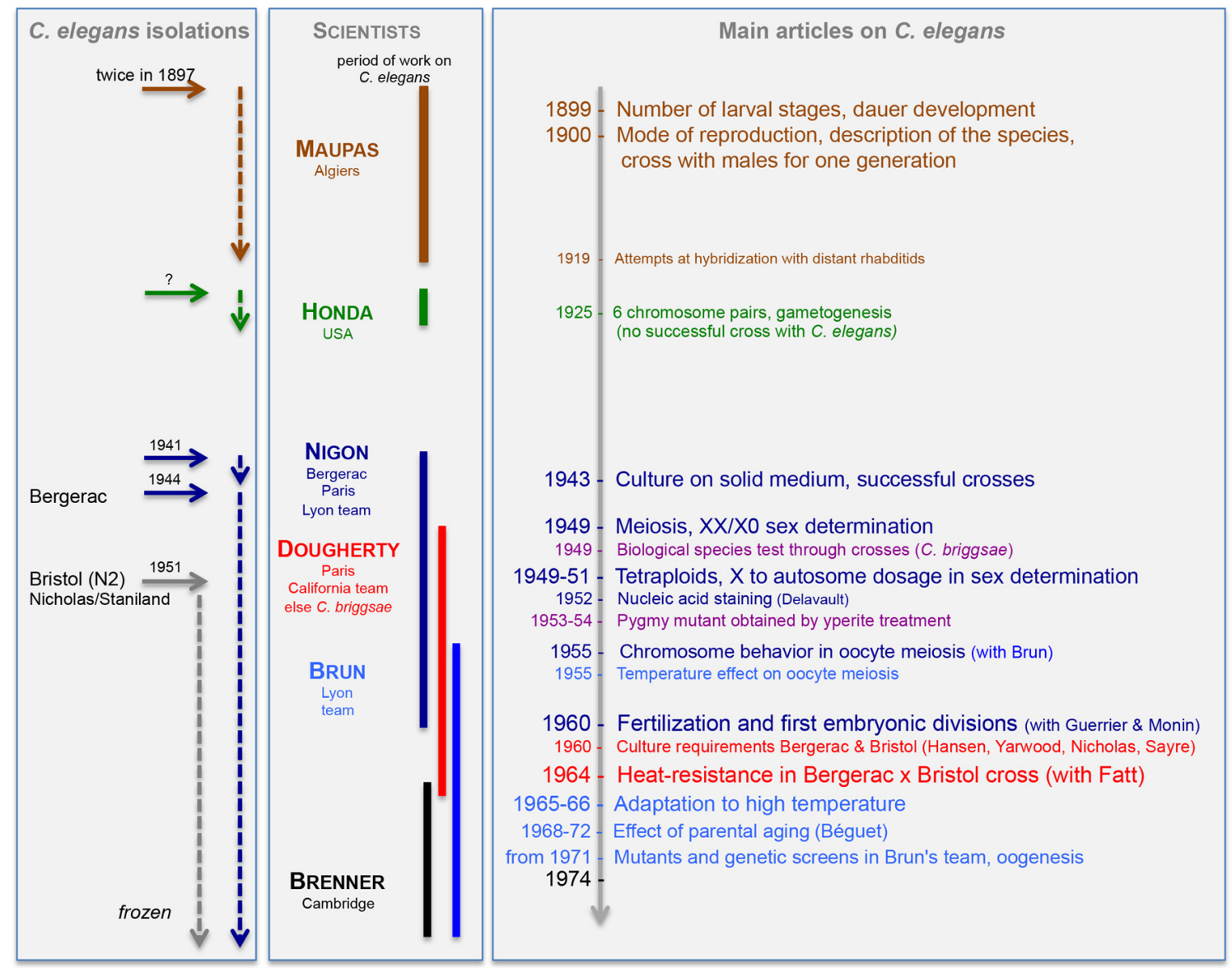

Figure 20. Outline of laboratory studies using C. elegans prior to 1974. Work by each main author is color-coded. Common work by Nigon and Dougherty is in purple.

By reviewing earlier work (Figure 20), we attempted to correct the modern misrepresentation of C. elegans research as originating with Brenner's work. In this section, we try to provide a brief account of the problems Nigon and Dougherty encountered in the 1950s and of Brenner's contribution in the context of previous work. The problems can be schematically divided as: 1) how can classical genetics be used to study formation of phenotypes in an animal?, and 2) how could one access the molecular level in an animal?

\subsection{C. elegans as a genetic model system}

\subsubsection{The pioneers in Caenorhabditis genetics}

After the early work of Gregor Mendel in the $19^{\text {th }}$ century with plants, the scientific field of genetics arose during the first half of the 20th century in model organisms such as Drosophila, Neurospora, mice, and various plants. First limited to the transmission of external characters, the first geneticists constructed abstract notions of heredity and genes. Morgan and his collaborators made an advance towards physical location by building a (still abstract) map of genes along Drosophila chromosomes. A first link between genes and phenotypes came with the works of Boris Ephrussi, Beadle, and Tatum and their "one gene, one enzyme" theory, which greatly influenced Dougherty and Nigon. The physical involvement of nucleic acids in genetic transmission was solved by studies of the genetics of bacteria and viruses, and of the structure, synthesis, and regulation of nucleic acids and proteins in the years 1940-1960. The gene appeared then, for the first time, as a chemically and biologically defined entity, the structure and activity of which could be simultaneously accessed through biochemical and genetical analyses. This anchoring of the gene in the biochemical realm contrasted with the abstract notion of heredity of the Weismannian tradition. 


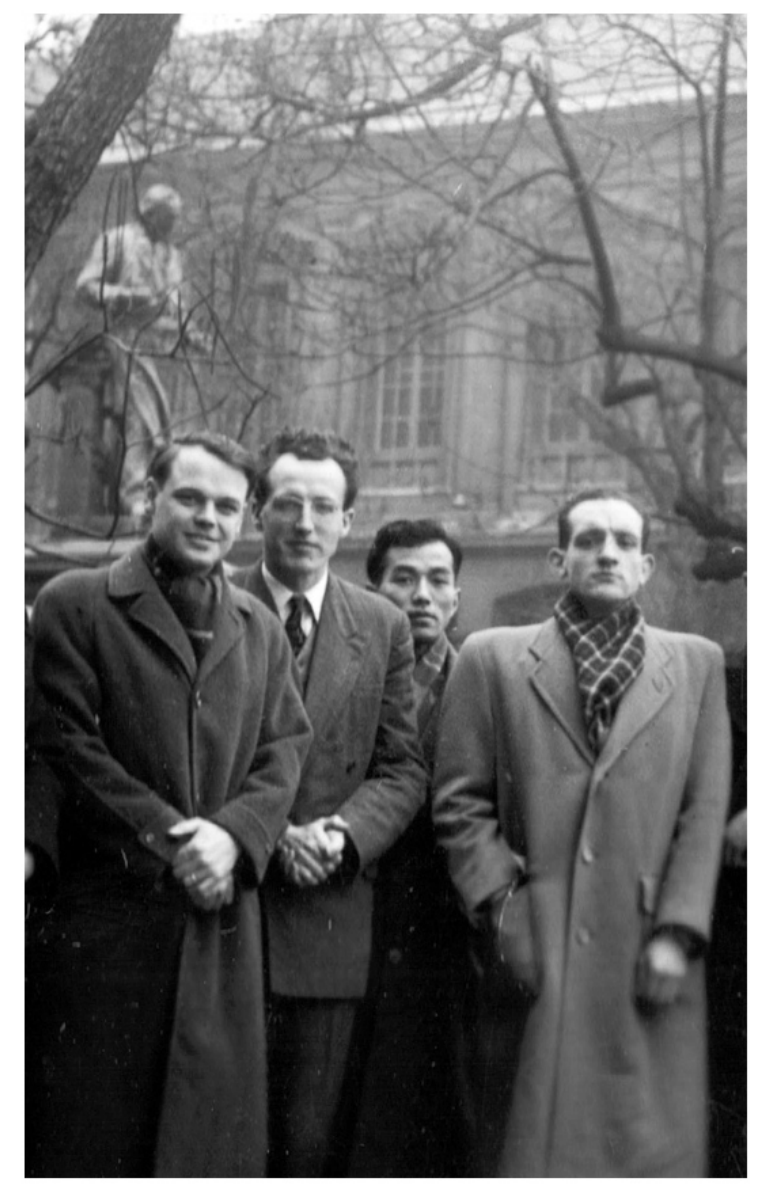

Figure 21. The pioneers. E. C. Dougherty, V. M. Nigon, a visitor, and J.-L. Brun, in front of the Claude Bernard University in the center of Lyon, 1952. Collection V. M. Nigon.

How could the nematode contribute to this modern biology? Was metabolism the entry point to experimental genetics, beyond the genetics of morphology that was practiced until then? In French laboratories, often refractory to the older abstract concept of the gene, these developments were provoking endless discussions. Opponents were scoffing at the "faith" of innocents who imagined they could succeed in manipulating these molecular chains, at the resolution limit of the most powerful electron microscopes. Nigon was one of these innocents, imagining that the fast multiplication and simple structure of nematodes would present the same kind of advantages as those provided by bacteria. The recruitment of experimental geneticists, embryologists, and biochemists to his team appeared an absolute necessity. Nigon, as a student, had first hoped to work in Paris at the Pasteur Institute, but the war context had retained him far from Paris. After the war, political circumstances prevented him (a member of the French Communist Party) from traveling to the United States (then in the period of McCarthy's anti-communist hunt) to widen his horizons. Nigon held Ephrussi in high esteem, but Ephrussi did not encourage his C. elegans project: in 1947 he asked Nigon when he would stop amusing himself with his little animals ("quand aurez-vous fini de vous amuser avec vos petites bêtes?"). André Lwoff was then relatively isolated, with widespread recognition in France coming only after the Nobel Prize in 1965. Thus, French science politics at the time was not favorable. In California, Dougherty made similar proposals on the potential of free-living nematodes, in his case extrapolating from the experiments of Beadle, but also had trouble securing funding in the context of his work on a nutritional medium (Ferris and Hieb, 2015). Unfortunately, Nigon and Dougherty were not advised nor heeded. In addition, the time was perhaps too early for developing an animal model organism for molecular biology, when the foundations of molecular biology were still being built.

Nigon, Dougherty, and Brun however made pioneering attempts in Caenorhabditis genetics and were the first to discover mutations in C. briggsae and C. elegans. It now appears clearly that several problems affected Nigon and Dougherty in the 1950s and prevented them from carrying further genetic experiments. Their inexperience made them search for mutations affecting characters with deep biological significance. And to neglect, hardly seeing them, 
variations that seemed to them of no interest. Their reference was the variety of Drosophila morphological phenotypes (color and shapes of eye, wing, etc.) and of Neurospora metabolic mutants. Spotting new phenotypes is not obvious, and greatly improves with self-confidence and training (Kohler, 1994; Brenner, 1994). Let us also note that behavioral genetics with the screening for such mutants in C. elegans and in Drosophila was a revolution of the late 1970s, which was not immediately accepted. So, on one hand, Nigon and Dougherty paid insufficient attention to various phenotypes, such as behavioral or developmental phenotypes; on the other hand, they did not conceive of the idea of using several mutations with the same phenotype. Testing whether mutations with the same phenotypes affected the same genetic locus with a genetic complementation test was not obvious at that time. A complex version of the complementation test, the cis-trans test of Seymour Benzer in the T4 phage and Ed Lewis in Drosophila, was just appearing; the modern version (without the cis arrangement of the two alleles, very difficult to obtain in most organisms including C. elegans) only became used in the 1960s (Hawley and Gilliland, 2006). Thus, the lack of diverse phenotypes was a critical obstacle for Nigon who could not see how to go further with the genetics of $C$. elegans, while Dougherty continued his quest for a defined medium to screen for metabolic mutants in the 'one gene, one enzyme' framework.

In Lyon, an important change occurred with the construction of a new laboratory, in part through the administrative supervision of Nigon. The old Faculty of Sciences, dating from 1880, was abandoned, and this new building opened in October 1962, with well-equipped spaces dedicated to experimental biology. This new start could have been placed under the joint authority of Nigon, Dougherty and Brun, for example, and be dedicated to the study of Rhabditis. The question was not even raised. It is likely that at that time, the scientific paths of these three scientists were too far engaged in independent routes to think of a new common start. Nigon abandoned nematodes rapidly and, after a long exploratory work on various species and various transmissible characters, found a more promising way towards molecular biology in the study of polysomes in Euglena and of human globin mutations. Among his collaborators, only Brun persisted, with extreme courage and loneliness, in pursuing studies on C. elegans, most of which ran against the contemporary mainstream of genetics.

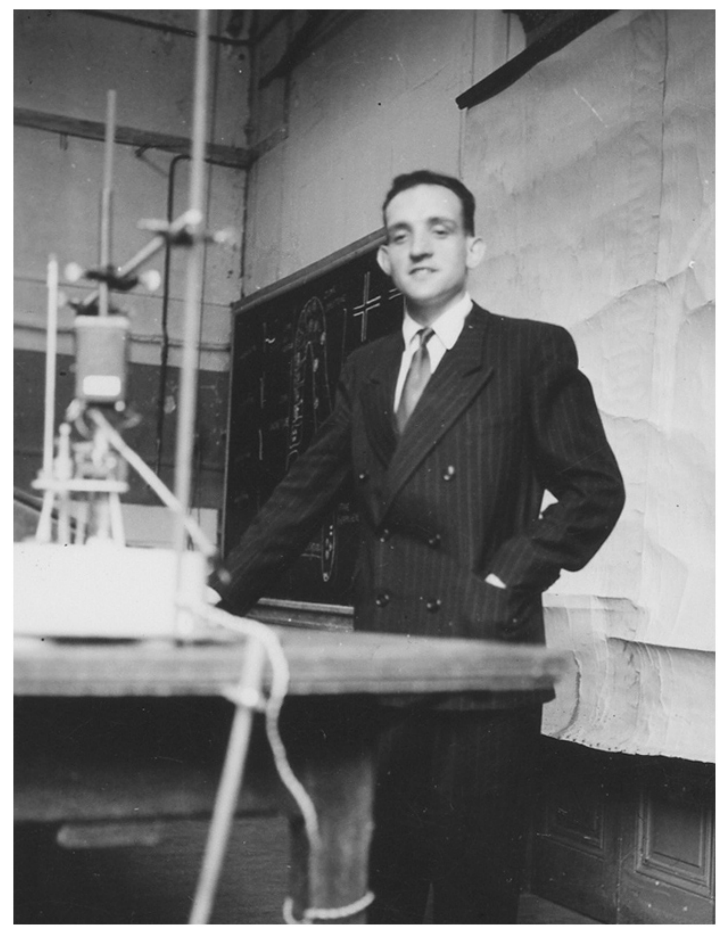

Figure 22. Jean Brun next to a highly precise temperature incubator for $\boldsymbol{C}$. elegans cultures. (PhD defense, 1966). The temperature-control system on the left was usually further placed in a temperature-controlled incubator with cooling, at a temperature of $22^{\circ} \mathrm{C}$. Note the drawing of the $C$. elegans hermaphrodite gonad and meiosis on the blackboard. Courtesy of Mrs. G. Brun.

\subsubsection{Bacteria geneticists turn to $C$. elegans in the mid-1960s}

In the early 1960s, Sydney Brenner was already famous for his work in the new and exciting field of molecular biology, particularly the discovery of messenger RNA and the genetic code (Judson, 1996; Brenner, 2001). In contrast to Nigon and Dougherty, Brenner had extensive experience with genetic analysis of bacteria and 
phages, where multiple mutations in various loci were known to have similar phenotypes, at least superficially, and he followed closely the phage experiments of Seymour Benzer. Like other molecular biologists, Brenner thought that the major questions had been solved in molecular biology by the early 1960s and he was searching for an adequate model animal to start a new research program in developmental biology and neurobiology. At that time, for similar reasons, Seymour Benzer turned to Drosophila behavior, Gunther Stent to the planarian, François Jacob to the mouse, etc. Brenner first experimented with a variety of small animals, including rotifers, and got particularly interested in nematodes. One aspect that strongly attracted him to the nematodes was the known invariance of their cellular composition-including neuronal connections for which he cited Goldschmidt-and their small size, allowing them to fit entirely in the electron microscope. Having an intermediate phenotypic level between genetic loci and behavior at the level of neurons and neuronal connections appeared to him as the way forward in neurobiology (Brenner, 2001).

Was Brenner much influenced by prior studies and scientists in its choice and use of C. elegans? Brenner asked Dougherty for the C. elegans Bristol culture in the fall of 1963, perhaps after seeing the article of Fatt and Dougherty (1963). His letter acknowledging receipt enthusiastically stated: "It is an astounding organism" (Dougherty, 1963). He then met Dougherty in Berkeley in 1965, while Brenner was on sabbatical (Stent, 1965; Brenner, 2001). Brenner however continued to experiment with many nematode species, and isolated and gathered many new strains. Besides the ease of culture and the facility for genetics, one key criterion that Brenner looked for was a good fixation for electron microscopy, so as to be able to reconstruct all neuronal connections. The first attempt at fixation of $C$. elegans was disastrous, due to the fact that the culture had already starved and entered the dauer stage, which does not fix well (Emmons, 2015; J. Hodgkin, personal communication). Therefore, despite the fact that $C$. elegans was easy and fast to grow and had the right reproductive mode, it was not Brenner's favorite at first. However, after a later successful attempt at electron microscopy on non-dauer animals, Brenner finally settled on C. elegans in 1967, producing his first mutant at the end of the year (Brenner, 1966/1981; Brenner, 2001; Brenner, 2009). Among free-living nematode species, C. elegans was the favorite species of both Maupas and Nigon, and among the species they studied, is indeed one of the fastest growing at the individual and population levels, and most transparent for microscopic observations. That Brenner converged on the same species, in great part independently after intensive searches and tests with his technicians Muriel Wigby and Nichol Thomson (Brenner, 1966/1981; Brenner, 2001), is not particularly surprising. Besides the choice of the species, Brenner corresponded with Dougherty, then Nigon, who sent him strains and techniques, for example for crosses (Dougherty, 1963; Nigon, 1966/1970). But altogether, Brenner's choice of C. elegans was made after a thorough examination of the properties of many other free-living nematode species (identified as Rhabditella, Rhabditis, Pellioditis, Pelodera, Mesorhabditis, Panagrolaimus, Panagrellus, Rhabditophanes, Acrobeloides, and Mesodiplogaster/Pristionchus) (Brenner, 1966/1981; Brenner, 2001).

Other molecular biologists became interested in C. elegans, inspired by Brenner. One who successfully continued was Gunter von Ehrenstein in Göttingen, Germany who contributed to the early cell lineage and developmental biology (Deppe et al., 1978; Schierenberg et al., 1980; Miwa et al., 1980; Laufer and von Ehrenstein, 1981; Cassada et al., 1981; Isnenghi et al., 1983). Two others started but did not pursue: François Jacob at the Institut Pasteur in Paris (Jacob, 1969; Jacob, 1970; de Chadarevian, 1998) and Marshall Nirenberg at the NIH (Nirenberg and Tabery, 2001). Besides being famous for his studies of lysogeny and the lactose operon, François Jacob was a close colleague of Sydney Brenner, with whom he had performed the famous experiment demonstrating the synthesis of messenger RNAs (Judson, 1996; Brenner, 2001). By the mid-1960s (the Nobel Prize was awarded to him in 1965), Jacob was looking for an animal model organism to study development. Influenced by Brenner, who sent him a culture (Jacob, 1997), he started work on C. elegans, and contacted Nigon about it. He successfully produced mutants of different types (dumpy, uncoordinated, roller, molt-defective, and deformed-head mutants) (Jacob, 1969; Jacob, 1970). The reasons Jacob gave for stopping his work on C. elegans were of two kinds. First, $C$. elegans was difficult to manipulate for embryological studies as it exploded upon introduction of a needle and the egg shell prevented the penetration of chemicals (Jacob, 1970). C. elegans classical embryological studies are indeed difficult but not impossible (Nigon, 1965; Laufer et al., 1980; Laufer and von Ehrenstein, 1981; Priess and Thomson, 1987; Wood, 1991; Goldstein, 1992); and injection into the germ line is now routinely performed for transgenesis (Mello and Fire, 1995). Second, in line with the aims of the Pasteur Institute, Jacob preferred to work on an organism closer to humans, and was also interested in studying cancer (Jacob, 1997). He thus turned to the mouse and cultured cell lines. It also appears that the (friendly) competition with Brenner was a factor in avoiding $C$. elegans.

The other famous molecular biologist to turn only briefly to C. elegans following Brenner was Marshall Nirenberg, a competitor of Brenner on deciphering the genetic code. Nirenberg also wanted to study neurobiology. A former student of Dougherty, Ruth Pertel came to his lab and produced locomotion and nervous system mutants 
of $C$. elegans. However, also fearing the competition with Brenner, he preferred to focus on his second line of research, neuroblastoma (Nirenberg and Tabery, 2001).

Brenner was working at the Laboratory of Molecular Biology in Cambridge, financed by the MRC, an exceptional place in terms of scientific level, funding, and freedom, where for many years he shared an office with Francis Crick. Postdocs came to study transfer RNAs, but often, convinced by Brenner's enthusiasm, ended up switching to the nematode (Brenner, 2001). This contrasted with the difficulties and resistance that both Nigon and Dougherty experienced to secure funding on their nematode projects. A key strength of Brenner was to be able to attract with his vision and aura both continued funding (despite low initial productivity in terms of papers) and a group of impressive individuals.

The first experimental paper of Brenner on C. elegans, published in 1974 (Brenner, 1974), is the genetic map, built from 300 mutants defining about 100 genes. The main classes were dumpy and uncoordinated mutants, as well as few other types, such as roller, long, blistered cuticles, and variably abnormal (head deformation). Interestingly, Drosophila scientists assign each locus a specific name corresponding to the phenotype of the mutant (or even with several names corresponding to the diversity of phenotypes for different alleles at a locus). Instead, Sydney Brenner and the early worm geneticists, facing the monotony of dumpys and the somewhat greater variety of locomotion phenotypes (uncoordinated, or Unc) opted for using the same phenotype name for many loci and numbering them: $d p y-1, d p y-2 \ldots$ up now to $d p y-31$; unc-1... unc-132, etc. (Brenner, 1974; Horvitz et al., 1979). This difference reflects both the systematic and abstract style of research that was adopted by $C$. elegans practitioners, as well as the paucity of obvious phenotypes. It also epitomizes the immediate need for complementation tests by the $C$. elegans geneticist.

Regarding phenotypes, Brenner et al. spotted and isolated other types of mutants, especially those producing bags of larvae. It was known for a long time that adult hermaphrodites could fail to lay their fertilized embryos and be devoured by their progeny hatching within the uterus. Maupas in particular gives vivid descriptions of the process (Maupas, 1900). In the Brenner lab conditions, with abundant food for the worms, this phenomenon did not occur with their wild-type strain. Only mutants with defective egg-laying appeared as bags of larvae and they were actively screened for in Cambridge and later in the laboratory of Robert Horvitz (Horvitz and Sulston, 1980; Trent et al., 1983). When analyzed at the tissue level, these mutations could affect either the egg-laying behavior, the formation of the neurons or muscles necessary for it, or the formation of the vulva, which is the epithelial structure allowing for egg-laying. In addition, a class of mutants with additional bumps on the ventral cuticle, named multivulva, corresponded to an excess of vulval formation. These bag-of-worms and multivulva mutants led the way to developmental genetics in C. elegans, while the uncoordinated (together with mutants resistant to nematicide drugs) led to the genetics of neurobiology.

For both development and neurobiology, the genetic analysis was accompanied by a heroic effort of the Brenner recruits towards an extensive, often complete, description of $C$. elegans at the cellular level. For development, Sulston and a small group of collaborators determined the complete cell lineage from egg to adult-an amazing feat (Sulston and Horvitz, 1977; Sulston et al., 1983; Kimble and Hirsh, 1979). They confirmed eutely and the quasi-invariance of development among individuals. As far as neurobiology was concerned, John White, with Nichol Thompson and Eileen Southgate devoted 20 years to achieve the complete neuron connection map (White et al., 1986; Emmons, 2015) and other aspects of the worm anatomy. The development of laser ablation of cells by White allowed testing the role of a cell in development or behavior (Sulston and White, 1980).

This systematic effort alleviated the belatedness of $C$. elegans as a model organism (e.g., compared to Drosophila melanogaster, which Morgan adopted in the 1900s). In addition, Sulston succeeded in cryopreserving $C$. elegans stocks, a key advantage over flies. Together with $C$. elegans" many other advantages, this 20 -year long effort of a small number of scientists allowed C. elegans to become by the 1990s one of the main model organisms in biological research. Hundreds of laboratories have since adopted it, many deriving in direct lineage from Brenner (some also adopted C. elegans independently), and form a close community sharing tools, a common database (Wormbase.org), meetings and informal communications.

\subsection{The advent of molecular biology with C. elegans}

Early on, Nigon was fascinated by the molecular biology of nucleic acids and was convinced that molecular genetics was the future of genetics. Inspired by the works by Jean Brachet and Oswald Avery, he knew that a relationship between the nucleic acids and a product of their activity must be sought. But it was unclear how to apply these notions to the genetics of eukaryotes, and likely it was much too early. Robert Delavault, a colleague 
History of research on C. elegans and other free-living nematodes as model organisms

and collaborator in Paris, tried to stain and visualize nucleic acids of several species of free-living nematodes (Delavault, 1952a; Delavault, 1952b; Delavault and Nigon, 1952). Later the Nigon team in Lyon succeeded in radioactive labeling of nucleic acids of various organisms. Jacqueline Nonnenmacher-Godet, after initial work in Nigon's laboratory, went to Dougherty's laboratory and applied the radioactive labeling technique to $C$. briggsae DNA (Nonnenmacher-Godet and Dougherty, 1964).

The molecular biology of the 1950-60s was built on methodological approaches such as crystallography, bacterial and phage genetics, and biochemistry, and not on what we now call "molecular biology" techniques. These techniques only came in the 1970s with the discovery of restriction enzymes and the possibility of cloning DNA. Nigon participated in this work by studying greening of bleached Euglena and thalassemic hemoglobin mutations in humans, leading him to the evolution of primate hemoglobins and the study of aviary viruses carrying erythroblastosis genes. Brenner, despite being a main actor in the development of molecular biology, embraced the genetics of $C$. elegans in the 1960s without yet knowing how molecular biology could be brought to development and neurobiology studies. The cellular level was the first anchor, but Brenner aimed at the molecular level for the future. Together with the first genetics paper of 1974 (Brenner, 1974), Sulston and Brenner published a lesser known article where they quite correctly assessed the size and complexity of the C. elegans genome using DNA reassociation kinetics (their estimation was $80 \mathrm{Mb}$ for what we now know is $100 \mathrm{Mb}$ ) (Sulston and Brenner, 1974). But the way forward was not obvious. Brenner had considered the possibility of isolating nutritional mutants, as advocated by Dougherty, as a possible route to clone genes (Brenner, 2001). He also considered complementing Bacillus subtilis mutants with $C$. elegans DNA to identify conserved genes (which we now know would not have worked because of introns) (Brenner, 2001). In the end, the first molecular identification of a C. elegans mutation used a biochemical approach: the abundance of muscle proteins allowed the identification of the unc-54 gene, as encoding a myosin heavy chain (Epstein et al., 1974).

Brenner reported: "Jim Watson, in particular, said he would not give me a penny for this work, complaining that I was 20 years ahead of my time. What changed everything was the advent of cloning and sequencing. It opened up all of genetics and gave us direct access to genes." (Brenner, 2009). As Watson had supposed, molecular biology indeed only developed fully 20 years after Brenner started, when mutated genes could be identified and rescued. Nigon's work was thus 40 years too early. Ironically, the way towards molecular biology is what the Bergerac strain became most known for to $C$. elegans scientists of the 1980-90s. The first transposon type, called Tc1, was discovered through a restriction length polymorphism between Bergerac and Bristol in Southern blots hybridized with different probes (Emmons et al., 1979). The high number of transposons and the transposition activity in the Bergerac strain then served as tools for gene identification (Emmons et al., 1983; Liao et al., 1983; Moerman and Waterston, 1984; Eide and Anderson, 1985; Plasterk and van Luenen, 1997; Transposons in C. elegans). The hundreds of Tc1 transposons were used for genetic mapping and identification of mutated genes (Finney et al., 1988; Ruvkun et al., 1989; Williams et al., 1992). Moreover, the high transposition rate, at least of the RW7000 subclone of Bergerac, could be used as a mutagen that rendered gene identification easier (Moerman and Waterston, 1984; Greenwald, 1985; Moerman et al., 1986).

In the spirit of the cell lineage or the nervous system connectome, a physical map of the whole genome was built through a new heroic enterprise by Sulston, together with Alan Coulson and Robert Waterston. They cloned the C. elegans genome in cosmids and yeast artificial chromosomes, building a physical map, which was progressively aligned with the genetic map (Coulson et al., 1986; Coulson et al., 1988; Coulson et al., 1995). This led C. elegans to be the first animal to have its genome fully sequenced, from ordered clones (The C. elegans Sequencing Consortium, 1998) as a way towards the human genome sequence to which Sulston, Coulson, and Waterston then contributed (Sulston and Ferry, 2002).

\section{Epilogue}

The development of tools and a body of knowledge in a single species (the 4th phase of studies in our introduction) was undoubtedly the road to fundamental biological discoveries. $C$. elegans proved a terrific model organism for genetic analysis of a wide variety of molecular, cellular, developmental, physiological, and behavioral questions and remains so. On the other hand, by focusing on a single species and a single reference strain, other aspects of biology could not be studied: first, those concerning evolution and diversity; second, biological features that are not present in $C$. elegans. Analyzing the many key discoveries that have been made using $C$. elegans is beyond the scope of this article. Instead we point below to several research directions that were primed by pre-Brenner studies and that seem to deserve in the future renewed or continued attention. Especially, we can now come back and use the amazing diversity of free-living nematodes to answer a variety of new questions pertaining to diversity in evolution. 


\subsection{At the core of genetics: reproduction, sex determination, meiosis, and polyploidy}

The initial interest in nematodes of early researchers, such as Boveri, Bütschli, van Beneden, Maupas, Krüger, P. Hertwig, and Belar, was triggered by the diversity of reproductive modes, based on the cytological diversity of meiosis and fertilization events. After Nigon determined the XX/X0 chromosomal basis of sex determination in $C$. elegans and correctly inferred that the ratio between $\mathrm{X}$ chromosomes and autosomes was the key, sex determination mechanisms were further studied in $C$. elegans using genetic screens, initially by Hodgkin and Brenner (Hodgkin and Brenner, 1977; Hodgkin, 1988; Hodgkin, 2002b). Single mutations could either transform XX animals into males (tra), or X0 animals to females (fem) or hermaphrodites (her), or be lethal specifically for X0 animals (xol). The analysis of these mutants showed that a low ratio of $\mathrm{X}$ chromosomes to autosomes in males activates expression of the xol-1 gene and downstream production of the HER-1 intercellular signaling molecule, which represses the TRA-2 receptor and starts a cascade of signaling events in each cell throughout the animal. The ratio between $\mathrm{X}$ chromosomes and autosomes is now known to be assessed by several $\mathrm{X}$-linked and autosomal elements that were identified by Barbara Meyer et al. (Somatic sex determination).

Concerning the partial independence between soma and germ line, some sex determination mutations called fog only feminize the hermaphrodite germ line. They transform XX hermaphrodites into true females without spermatogenesis, thus dissociating the mechanisms of sexual differentiation of the gonad from that of the gametes, as predicted by Nigon. Analysis of these mutants led to the understanding that the wild-type hermaphrodite germ line differentiates successively as male and female through a temporal molecular switch from spermatogenesis to oogenesis: the action of the TRA-2 receptor is first inhibited by FOG-2 (sperm formation) before being reactivated (Sex determination in the germ line).

From the knowledge of sex determination pathways, it became possible to manipulate at will the sex determination mechanisms of $C$. elegans using mutants. Transition between a hermaphrodite selfing mode of reproduction and a gonochoristic reproduction can be easily achieved: mutants in a single gene, such as fog-2, reproduce through females and males (Schedl and Kimble, 1988). Hodgkin could further produce all kinds of situations, including maternally or environmentally determined sex or other forms of chromosomal sex determination (Hodgkin, 2002a).

Once molecular details became clearer in C. elegans, in the last 20 years the evolution of sex determination and of the modes of reproduction in nematodes became again a center of interest (Pires-daSilva, 2007). As inferred by the older authors, the transformation of a male-female species into a hermaphroditic one may be easy in terms of mutational steps. In the gonochoristic $C$. remanei, mutations in only two independent pathways are sufficient to create hermaphroditic nematodes (Baldi et al., 2009). The Caenorhabditis genus is presently known to harbor three independent transitions to selfing (Kiontke et al., 2011; Thomas et al., 2012). An evolutionary consequence of these transitions is a great reduction in molecular diversity and the appearance of outbreeding depression (the progeny or grand-progeny is less fit than the parents), contrasting with the observed inbreeding depression in gonochoristic nematodes (Dolgin et al., 2007; Gimond et al., 2013). Recessive deleterious mutations can indeed be maintained at low frequencies in gonochoristic species, whereas they become rapidly exposed in a homozygote combination in selfing species and are then efficiently purged by natural selection. The evolutionary maintenance of males remains a key question under active study (Anderson et al., 2010). Spontaneous changes in mode of reproduction from the male-female mode (such as in P. pellio, with pseudogamy through males, Section 5.2.2; or in P. lheritieri, Section 3.5) have not been observed again to our knowledge. They could be looked for.

In a thread running from Boveri, Maupas, and Nigon to recent work in C. elegans (Rog and Dernburg, 2013; Lui and Colaiacovo, 2013), nematodes have been particularly instrumental in studies of meiosis. Now chromosomes or chromosome segments can be individually marked, not only through genetic markers, but also using fluorescent probes (Nabeshima et al., 2011). Homologs find each other at the onset of meiosis through pairing at foci on the nuclear membrane, with different proteins assembling the different chromosomes (Phillips and Dernburg, 2006; Rog and Dernburg, 2013). These pairing centers are anchored at the nuclear membrane through the ZYG-12 protein (that with a missense mutation in Bergerac) (Sato et al., 2009). The sites of cross-overs can be marked and the regulation of cross-overs after double-stranded break formation is increasingly understood (Rog and Dernburg, 2013; Vader and Musacchio, 2014). How these meiotic processes change during evolution is now a promising field of investigation.

Among easily culturable members of the family Rhabditidae alone a wide variety of modes of reproduction with variations in meiosis and fertilization are known: hermaphrodite-male, female-male, parthenogenetic 
Diploscapter spp.), alternating generations (Rhabditis sp. SB347), pseudogamous hermaphrodite (Rhabditis anomala and $R$. aberrans, to be found again, possibly in frozen collections as "hermaphrodites"), and partially pseudogamous females and males (Mesorhabditis belari; Mesorhabditis sp. PS1179). This diversity is a great tool to study genetic mechanisms underlying such evolutionary switches, and their evolutionary consequences. In contrast to the evolutionary transition to selfing, transitions towards parthenogenesis or pseudogamy affect basic aspects of meiosis and fertilization, which remain to be studied. How do the cellular events leading to the evolution of pseudogamy, parthenogenesis, or alternate generations evolve? How is the alternative between pseudogamy and true fertilization controlled in the egg of M. belari?

A further open field concerns the partial stability of tetraploids in C. elegans. Questions concern: 1) the problem of dosage compensation between chromosomes in unusual chromosome formulas, and 2) meiotic pairing and progression in a tetraploid situation.

In conclusion, the diversity in modes of reproduction and meiosis is a historical legacy of research in nematodes, which can be explored in terms of molecular regulation and evolution of cell biological processes, as well as for its impact on evolutionary dynamics.

\subsection{Progressive multigenerational sterility and acclimatization}

Brun's experiments of laboratory adaptation of C. elegans to high temperature (Section 10) were unconventional adaptation experiments because he used a very low population size-1-10 individuals per generation. Natural selection is efficient at high population size. In addition, when starting, as Brun did, with a population harboring no starting genetic diversity, a large population size also allows for incoming genetic variation through new mutation. Thus, the use of a low population size may be appropriate in the case of non-genetic acclimatization but not a design for standard genetic adaptation experiments.

The use of $C$. elegans in experimental evolution has started anew in the last 15 years, along the lines of standard population genetics (Gray and Cutter, 2014). The mode of reproduction and freezing ability of C. elegans are great assets. Experiments of adaptation to novel environments have included pathogens (Morran et al., 2009; Schulte et al., 2010), high salt (Theologidis et al., 2014), as well as high temperature for C. elegans (Gainutdinov et al., 2007) and C. remanei (Sikkink et al., 2014). Experiments at a small population size have also been performed. A population size of one corresponds to the standard design of mutation accumulation experiments, aimed at assessing the rate and distribution of mutational effects, most of them deleterious. Mutation accumulation at a population size of one indeed results in a fitness decay (Vassilieva et al., 2000; Keightley et al., 2000). However, a population size of 10 basically alleviates deleterious effects of mutation accumulation (Estes et al., 2004). A reexpansion at high population size of low fitness lines allows for surprisingly rapid recovery (Estes and Lynch, 2003).

A first exciting prospect concerns the mechanistic and evolutionary aspects of the progressive temperature-sensitive sterility observed by Brun. Although it may differ from that in Bergerac, progressive sterility at $25{ }^{\circ} \mathrm{C}$ is common among C. elegans wild isolates (M.-A. Félix, unpublished). The molecular and cellular basis of this progressive sterility and its evolutionary significance in the regulation of transgenerational memory and in adaptation remain to be studied.

A second question concerns the importance of prior non-genetic acclimatization in evolution, as opposed to direct genetic (DNA-based) adaptation. Brun concluded that the adaptation he observed was of a non-genetic nature. Although this possibility has been discounted by the synthetic theory of evolution in the mid-20th century, recent research on C. elegans and other organisms has uncovered molecular mechanisms whereby gene expression changes (potentially induced by the environment) could be passed on to the progeny for several generations. This includes small RNAs in C. elegans, which have been shown to be involved in physiological (same-generation) response to the environment (Félix et al., 2011; Ashe et al., 2013; Juang et al., 2013), and may be passed on to the progeny (Grishok et al., 2000; Alcazar et al., 2008; Burton et al., 2011; Gu et al., 2012; Ashe et al., 2012). Recent demonstrations of multigenerational effects in a physiological wild-type context (Sterken et al., 2014; Rechavi et al., 2014; Remy, 2010; Schott et al., 2014) are interesting and remain to be reproduced by others. Their possible role in adaptation to the environment and their importance and interplay relative to genetic variation under different circumstances are exciting open questions. 


\section{Acknowledgements}

We are very grateful to Jonathan Hodgkin for encouragement and information, to Geneviève Brun, Nicole Mounier, Joëlle Starck, and Mona Abirached-Darmency for interviews and historical information on the Brun laboratory and to Howard Ferris for sending us Ellsworth Dougherty's Curriculum Vitae. We thank Bill Wood, David Hirsh, Mark Edgley, and Don Moerman for information on Bergerac history, and Aurélien Richaud for genotyping Bergerac strains. Many colleagues provided advice and material, particularly Abby Dernburg, Mark Viney, Marie Delattre, Kathrin Gieseler, Einhard Schierenberg, Adrian Streit, and Michel Morange. For helpful comments and corrections on the manuscript, we thank Marie Delattre, Mark Viney, Patrick Phillips, Jonathan Ewbank, Walter Sudhaus, and the reviewers. We also thank the Library of the Bibliothèque Inter-Universitaire de Santé in Paris and M. Sillet, and the Library of the Museum National d'Histoire Naturelle and its staff, who provided many articles. Victor Marc Nigon dedicates this last work to Professor Albert Vandel and to his own students and collaborators.

\section{References}

Abdul Kader, N., and Brun, J. (1976). Isolation of sterile or lethal temperature-sensitive mutants in Caenorhabditis elegans var. Bergerac. Nematologica 22, 222-226. Article

Abdulkader, N., and Brun, J.-L. (1978). Induction, detection and isolation of temperature-sensitive lethal and/or sterile mutants in nematodes. I. The free-living nematode Caenorhabditis elegans. Rev. Nematol. 1, 27-38. Article

Abdulkader, N., and Brun, J.-L. (1980). Caractéristiques génétiques et physiologiques de la thermosensibilité du développement embryonnaire chez un mutant à létalité conditionnelle de Caenorhabditis elegans, lignée Bergerac. Rev. Nematol. 3, 11-19. Article

Abdulkader, N., Gibert, M.-A., Starck, J., Bosch, C., and Brun, J.-L. (1980). Temperature-sensitive mutations in Caenorhaditis elegans: a sterile mutation affecting oocyte I core relations. Rev. Nematol. 3, 201-212. Article

Abi-Rached, M., and Brun, J.-L. (1975). Etude ultrastructurale des relations entre ovocytes et rachis au cours de l'ovogenèse du nématode Caenorhabditis elegans. Nematologica 21, 151-162. Abstract

Abirached, M., and Brun, J.-L. (1978). Ultrastructural changes in the nudear and perinuclear regions of the oogonia and primary oocytes of Caenorhabditis elegans, Bergerac strain. Rev. Nematol. 1, 63-72. Article

Abirached, M., and Brun, J. (1979). L'évolution du complexe synaptonématique dans le noyau ovocytaire en prophase méiotique de Caenorhabditis elegans (Nematoda). C. R. Acad. Sc. Paris D 288, 425-428.

Ahmed, S., and Hodgkin, J. (2000). MRT-2 checkpoint protein is required for germline immortality and telomere replication in C. elegans. Nature 403, 159-164. Abstract Article

Albertson, D.G., Rose, A.M., and Villeneuve, A.M. (1997). Chromosome organization, mitosis and meiosis. In $C$. elegans II, D.L. Riddle, T. Blumenthal, B.J. Meyer, and J.R. Priess, eds. (Cold Spring Harbor, NY: Cold Spring Harbor Laboratory Press), pp. 47-78. Article

Albertson, D.G., and Thomson, J.N. (1993). Segregation of holocentric chromosomes at meiosis in the nematode, Caenorhabditis elegans. Chromosome Res. 1, 15-26. Abstract

Alcazar, R.M., Lin, R., and Fire, A.Z. (2008). Transmission dynamics of heritable silencing induced by double-stranded RNA in Caenorhabditis elegans. Genetics 180, 1275-1288. Abstract Article

Anderson, J.L., Morran, L.T., and Phillips, P.C. (2010). Outcrossing and the maintenance of males within C. elegans populations. J. Hered. 101 Suppl 1, S62-74. Abstract Article

Ankeny, R. (2001). The natural history of Caenorhabditis elegans research. Nat. Rev. Genet. 2, 474-479. Abstract Article 
Ashe, A., Bélicard, T., Le Pen, J., Sarkies, P., Frezal, L., Lehrbach, N.J., Félix, M.A., and Miska, E.A. (2013). A deletion polymorphism in the Caenorhabditis elegans RIG-I homolog disables viral RNA dicing and antiviral immunity. eLife 2, e00994. Abstract Article

Ashe, A., Sapetschnig, A., Weick, E.M., Mitchell, J., Bagijn, M.P., Cording, A.C., Doebley, A.L., Goldstein, L.D., Lehrbach, N.J., Le Pen, J., et al. (2012). piRNAs can trigger a multigenerational epigenetic memory in the germline of C. elegans. Cell 150, 88-99. Abstract Article

Bagijn, M.P., Goldstein, L.D., Sapetschnig, A., Weick, E.M., Bouasker, S., Lehrbach, N.J., Simard, M.J., and Miska, E.A. (2012). Function, targets, and evolution of Caenorhabditis elegans piRNAs. Science 337, 574-578. Abstract Article

Balachandar, R., and Lu, N.C. (2005). Nutritional requirements for pantothenate, pantethine or coenzyme A in the free-living nematode Caenorhabditis elegans. Nematology 7, 761-766. Abstract

Baldi, C., Cho, S., and Ellis, R.E. (2009). Mutations in two independent pathways are sufficient to create hermaphroditic nematodes. Science 326, 1002-1005. Abstract Article

Barringer, B.C. (2007). Polyploidy and self-fertilization in flowering plants. Am. J. Bot. 94, 1527-1533. Abstract Article

Béguet, B. (1972). The persistence of processes regulating the level of reproduction in the hermaphrodite nematode Caenorhabditis elegans, despite the influence of parental aging, over several consecutive generations. Exp. Gerontol. 7, 207-218. Abstract Article

Béguet, B. (1978). Etude génétique d'un mutant méiotique dominant chez Caenorhabditis elegans, souche Bergerac. Rev. Nematol. 1, 39-45. Article

Béguet, B., and Brun, J.-L. (1972). Influence of parental aging on the reproduction of the F1 generation in a hermaphrodite nematode Caenorhabditis elegans. Exp. Gerontol. 7, 195-206. Abstract Article

Béguet, B., and Gibert, M.-A. (1978). Obtention d'un mutant hermaphrodite autofécond muni d'une bourse copulatrice mâle chez le Nématode libre Caenorhabditis elegans, lignée Bergerac. C. R. Acad. Sc. Paris D 286, 989-992.

Belar, K. (1923). Über den Chromosomenzyklus von parthenogenetischen Erdnematoden. Biol. Zbl. 43, 513-518.

Belar, K. (1924). Die Cytologie der Merospermie bei freilebenden Rhabditis-Arten. Z. f. Zellen und Gewebelehre 1, $1-21$.

Bento, G., Ogawa, A., and Sommer, R.J. (2010). Co-option of the hormone-signalling module dafachronic acid-DAF-12 in nematode evolution. Nature 466, 494-497. Abstract Article

Bessereau, J.-L. Transposons in C. elegans (January 18, 2006), WormBook, ed. The C. elegans Research Community, WormBook, doi/10.1895/wormbook.1.70.1,http://www.wormbook.org. Abstract

Article

Beukeboom, L.W., and Vrijenhoek, R.C. (1998). Evolutionary genetics and ecology of sperm-dependent parthenogenesis. J. Evol. Biol. 11, 755-782. Abstract Article

Bilgir, C., Dombecki, C.R., Chen, P.F., Villeneuve, A.M., and Nabeshima, K. (2013). Assembly of the synaptonemal complex is a hghly temperature-sensitive process that is supported by PGL-1 during Caenorhabditis elegans meiosis. G3 3, 585-595. Abstract Article

Blaxter, M.L., De Ley, P., Garey, J.R., Liu, L.X., Scheldeman, P., Vierstraete, A., Vanfleteren, J.R., Mackey, L.Y., Dorris, M., Frisse, L.M., et al. (1998). A molecular evolutionary framework for the phylum Nematoda. Nature 392, 71-75. Abstract Article 
Bonfig, R. (1925). Die Determination der Hauptrichtungen des Embryos von Ascaris megalocephala. Z. Wiss. Zool. $124,407-456$.

Bose, N., Ogawa, A., von Reuss, S.H., Yim, J.J., Ragsdale, E.J., Sommer, R.J., and Schroeder, F.C. (2012). Complex small-molecule architectures regulate phenotypic plasticity in a nematode. Angew. Chem. 51, 12438-12443. Abstract

Boulin, T. et al. Reporter gene fusions (April 5, 2006), WormBook, ed. The C. elegans Research Community, WormBook, doi/10.1895/wormbook.1.106.1, http://www.wormbook.org. Abstract Article

Boveri, T. (1887). Über Differenzierung der Zellkerne während der Furchung des Eies von Ascaris megalocephala. Anat. Anz. 2, 688-693.

Boveri, T. (1892). Über die Entstehung des Gegensatzes zwischen den Geschlechtszellen und den somatischen Zellen bei Ascaris megalocephala. Sitzber. Ges. Morph. Physiol. München 8, 114-125.

Boveri, T. (1899). Die Entwickelung von Ascaris megalocephala mit besonderer Rücksicht auf die Kernverhältnisse. G. Fischer, Jena. Festschrift zum siebenzigsten Geburtstag von Carl von Kupffer, 383-430.

Boveri, T. (1910). Die Potenzen der Ascaris-Blastomeren bei abgeänderter Furchung. Zugleich ein Beitrag zur Frage qualitativ-ungleicher Chromosomen-Teilung. G. Fischer, Jena. Festschrift zum sechzigsten Geburtstag Richard Hertwigs (München), 133-214.

Boveri, T. (1911). Über das Verhalten der Geschlechtschromosomen bei Hermaphroditismus. Beobachtungen an Rhabditis nigrovenosa. Verh. Phys. Med. Ges. 41, 83-97.

Brauchle, M., Kiontke, K., MacMenamin, P., Fitch, D.H., and Piano, F. (2009). Evolution of early embryogenesis in rhabditid nematodes. Dev. Biol. 335, 253-262. Abstract Article

Brenner, S. (1966/1981). 126. N Isolation. In CSHL Archives Repository (Cold Spring Harbor), Reference $\mathrm{SB} / 6 / 5 / 126$. Article

Brenner, S. (1974). The genetics of Caenorhabditis elegans. Genetics 77, 71-94. Abstract Article

Brenner, S. (1988). Foreword. In The Nematode Caenorhabditis elegans, W.B. Wood, ed. (Cold Spring Harbor, NY: Cold Spring Harbor Laboratory Press), pp. ix-xiii. Article

Brenner, S. (1994). Mendelian experiments with behavioural mutants. In Web of Stories, L. Wolpert, ed. http://www.webofstories.com/play/sydney.brenner/153. Accessed 4 April 2015.

Brenner, S. (2001). My life in science (London: BioMed Central Ltd). Article

Brenner, S. (2009). In the beginning was the worm. Genetics 182, 413-415. Abstract Article

Briggs Gochnauer, M., and McCoy, E. (1954). Response of a soil nematode, Rhabditis briggsae, to antibiotics. J. Exp. Zool. 125, 377-406. Abstract

Brown, A.E. (2003). In the beginning was the worm: finding the secrets of life in a tiny hermaphrodite (New York, NY: Columbia University Press).

Brun, J. (1955). Evolution de la prophase méiotique chez Caenorhabditis elegans Maupas 1900 sous l'influence des températures élevées. Bull. Biol. Fr. Belg. 89, 326-346.

Brun, J. (1965). Genetic adaptation of Caenorhabditis elegans (Nematoda) to high temperatures. Science 150, 1467. Abstract

Brun, J.-L. (1966a). L'adaptation aux températures élevées chez un Nématode: Caenorhabditis elegans Maupas 1900. L'adaptation et son évolution. Ann. Biol. Anim. Bioch. Bioph. 6, 127-158. Article 
History of research on C. elegans and other free-living nematodes as model organisms

Brun, J.-L. (1966b). L'adaptation aux températures élevées chez un Nématode Caenorhabditis elegans Maupas 1900. II.- Stabilité et physiologie de l'adaptation. Ann. Biol. Anim. Bioch. Bioph. 6, 267-300. Article

Brun, J.-L. (1966c). L'adaptation aux températures élevées chez un Nématode: Caenorhabditis elegans Maupas 1900. III.-Rôle des facteurs autres que la température dans le processus d'adaptation. Ann. Biol. Anim. Bioch. Bioph. 6, 439-466. Article

Brun, J.-L., and Lebre, D. (1968). Influence du vieillissement parental sur la fécondité des descendants de 1re génération chez un nématode hermaphrodite autofécond: Caenorhabditis elegans. C. R. Acad. Sc. Paris D 266, 2149-2152.

Buckley, B.A., Burkhart, K.B., Gu, S.G., Spracklin, G., Kershner, A., Fritz, H., Kimble, J., Fire, A., and Kennedy, S. (2012). A nuclear Argonaute promotes multigenerational epigenetic inheritance and germline immortality. Nature 489, 447-451. Abstract Article

Buecher, E.J., Hansen, E.L., and Yarwood, E.A. (1970). Growth of nematodes in defined medium containing hemin and supplemented with commercially available proteins. Nematologica 16, 403-409. Abstract

Bumbarger, D.J., Riebesell, M., Rodelsperger, C., and Sommer, R.J. (2013). System-wide rewiring underlies behavioral differences in predatory and bacterial-feeding nematodes. Cell 152, 109-119. Abstract Article

Burton, N.O., Burkhart, K.B., and Kennedy, S. (2011). Nuclear RNAi maintains heritable gene silencing in Caenorhabditis elegans. Proc. Natl. Acad. Sci. U. S. A. 108, 19683-19688. Abstract Article

Cadet, P., and Dion, M. (1973). Production of Caenorhabditis elegans mutants under the influence of ethyl-methane-sulfonate E.M.S. Nematologica 19, 117-118.

Cassada, R., Isnenghi, E., Culotti, M., and von Ehrenstein, G. (1981). Genetic analysis of temperature-sensitive embryogenesis mutants in Caenorhabditis elegans. Dev. Biol. 84, 193-205. Abstract Article

Chaudhuri, J., Kache, V., and Pires-daSilva, A. (2011). Regulation of sexual plasticity in a nematode that produces males, females, and hermaphrodites. Curr. Biol. 21, 1548-1551. Abstract Article

Collins, J.J., and Anderson, P. (1994). The Tc5 family of transposable elements in Caenorhabditis elegans. Genetics 137, 771-781. Abstract Article

Collins, J., Forbes, E., and Anderson, P. (1989). The Tc3 family of transposable elements in Caenorhabditis elegans. Genetics 121, 47-55. Abstract Article

Conine, C.C., Moresco, J.J., Gu, W., Shirayama, M., Conte, D., Jr., Yates, J.R., 3rd, and Mello, C.C. (2013). Argonautes promote male fertility and provide a paternal memory of germline gene expression in C. elegans. Cell 155, 1532-1544. Abstract Article

Coulson, A., Huynh, C., Kozono, Y., and Shownkeen, R. (1995). The physical map of the Caenorhabditis elegans genome. In Caenorhabditis elegans Modern Biological Analysis of an Organism, H.F. Epstein and D.C. Shakes, ed. (San Diego, CA: Academic Press), pp. 533-550.

Coulson, A., Waterston, R., Kiff, J., Sulston, J., and Kohara, Y. (1988). Genome linking with yeast artificial chromosomes. Nature 335, 184-186. Abstract Article

Coulson, A.R., Sulston, J., Brenner, S., and Karn, J. (1986). Toward a physical map of the genome of the nematode Caenorhabditis elegans. Proc. Natl. Acad. Sci. U. S. A. 83, 7821-7825. Abstract Article

Crook, M. (2014). The dauer hypothesis and the evolution of parasitism: 20 years on and still going strong. Int. J. Parasitol. 44, 1-8. Abstract Article

Darlington, C.D. (1937). Recent advances in cytology, 2nd ed. (Philadelphia: P. Blakiston's Son \& Co. Inc.).

Das, P.P., Bagijn, M.P., Goldstein, L.D., Woolford, J.R., Lehrbach, N.J., Sapetschnig, A., Buhecha, H.R., Gilchrist, M.J., Howe, K.L., Stark, R., et al. (2008). Piwi and piRNAs act upstream of an endogenous siRNA pathway to suppress Tc3 transposon mobility in the Caenorhabditis elegans germline. Mol. Cell 31, 79-90. Abstract Article 
Davitashvili, L.S. (1969). Deficiencies of the synthetic theory of evolution. Evolution 23, 513-516. Article

de Chadarevian, S. (1998). Of worms and programmes: Caenorhabditis elegans and the study of development. Stud. Hist. Phil. Biol. Biomed. Sci. 29, 81-105. Article

Delavault, R. (1952a). La teneur en acide désoxyribonucléique des noyaux sexuels chez un Rhabditis hermaphrodite. C. R. Acad. Sc. Paris D 234, 884-885.

Delavault, R. (1952b). Etude cytologique des acides nucléiques chez un Nématode libre (Rhabditis elegans Maupas 1900). Arch. Anat. Micr. 41, 41-68.

Delavault, R., and Nigon, V. (1952). L'évolution des acides nucléiques dans les cellules reproductives d'un nématode pseudogame. Arch. Biol. 63, 393-410. Abstract

Deppe, U., Schierenberg, E., Cole, T., Krieg, C., Schmitt, D., Yoder, B., and von Ehrenstein, G. (1978). Cell lineages of the embryo of the nematode Caenorhabditis elegans. Proc. Natl. Acad. Sci. U. S. A. 75, 376-380. Abstract Article

Dion, M., and Brun, J.L. (1971). Cartographie génique du Nématode libre Caenorhabditis elegans, variété Bergerac. Mol. Gen. Genet. 112, 133-151. Abstract Article

Dolgin, E.S., Charlesworth, B., Baird, S.E., and Cutter, A.D. (2007). Inbreeding and outbreeding depression in Caenorhabditis nematodes. Evolution 61, 1339-1352. Abstract Article

Dougherty, E.C. (1949). The rôle of free-living nematodes in genetic research. 8th International Congress of Genetics (suppl to Hereditas), 562-564. Article

Dougherty, E.C. (1950). Sterile pieces of chick embryo as a medium for the indefinite axenic cultivation of Rhabditis briggsae Dougherty and Nigon, 1949 (Nematoda: Rhabditidae) Science 111, 258. Abstract Article

Dougherty, E.C. (1953). The genera of the subfamily Rhabditinae Micoletzky, 1922 (Nematoda). In Thapar Commemoration Volume: A Collection of Articles Presented to Prof. G. S. Thapar on His 60th BIRTHDAY, 1953, J. Dayal, K.S. Singh, eds. (Lucknow, India: University of Lucknow, Department of Zoology), pp. 69-76.

Dougherty, E.C. (1959). Introduction to Axenic culture of Invertebrate Metazoa: a goal. Ann. NY Acad. Sci. 411, 27-54. Article

Dougherty, E.C. (1963). Brenner requests C. elegans, S. Brenner, ed. (CSHL Archives Repository), Reference SB/1/1/162. http://libgallery.cshl.edu/items/show/60759 Article

Dougherty, E.C., and Calhoun, H.G. (1948a). Possible significance of free-living nematodes in genetic research. Nature 161, 29. Abstract Article

Dougherty, E.C., and Calhoun, H.G. (1948b). Experiences in culturing Rhabditis pellio (Schneider, 1866) Bütschli, 1873 (Nematoda: Rhabditidae), and related soil nematodes. Proc. Helm. Soc. Wash. 15, 55-68. Article

Dougherty, E.C., Hansen, E.L., Nicholas, W.L., Mollett, J.A., and Yarwood, E.A. (1959). Axenic cultivation of Caenorhabditis briggsae (Nematoda: Rhabditidae) with unsupplemented and supplemented chemically defined media. Ann. NY Acad. Sci. 411, 176-217. Article

Dougherty, E.C., and Nigon, V. (1949). A new species of the free-living nematode genus Rhabditis of interest in comparative physiology and genetics. J. Parasitol. 35, 11.

Dougherty, E.C., and Nigon, V. (1953). The effect of acriflavine a mixture of 2,8-diaminoacridine and 2,8-diamino-10-methyl-acridinium-chloride on the growth of the nematode Caenorhabditis elegans. International Congress of Zoology. Copenhagen 14, 247.

Duerr, J. S. Immunohistochemistry (June 19, 2006), WormBook, ed. The C. elegans Research Community, WormBook, doi/10.1895/wormbook.1.105.1,http://www.wormbook.org. Abstract Article 
Egilmez, N.K., Ebert, R.H.I., and Reis, R.J.S. (1995). Strain evolution in Caenorhabditis elegans: transposable elements as markers of interstrain evolutionary history. J. Mol. Evol. 40, 372-381. Abstract

Eide, D., and Anderson, P. (1985). Transposition of Tc1 in the nematode Caenorhabditis elegans. Proc. Natl. Acad. Sci. U. S. A. 82, 1756-1760. Abstract Article

Ellis, R. and Schedl, T. Sex determination in the germ line (March 5, 2007), WormBook, ed. The C. elegans Research Community, WormBook, doi/10.1895/wormbook.1.82.2, http://www.wormbook.org. Abstract Article

Emmons, S. (2015). The beginning of connectomics: a commentary on White et al. (1986) 'The structure of the nervous system of the nematode Caenorhabditis elegans'. Phil. Trans. R. Soc. B 370, 20140309. Abstract Article

Emmons, S.W., Klass, M.R., and Hirsh, D. (1979). Analysis of the constancy of DNA sequences during development and the evolution of the nematode Caenorhabditis elegans. Proc. Natl. Acad. Sci. U. S. A. 76, 1333-1337. Abstract Article

Emmons, S.W., and Yesner, L. (1984). High-frequency excision of transposable element Tc1 in the nematode Caenorhabditis elegans is limited to somatic cells. Cell 36, 599-605. Abstract Article

Emmons, S.W., Yesner, L., Ruan, K.-S., and Katzenberg, D. (1983). Evidence for a transposon in Caenorhabditis elegans. Cell 32, 55-65. Abstract Article

Epstein, H.F., Waterston, R.H., and Brenner, S. (1974). A mutant affecting the heavy chain of myosin in Caenorhabditis elegans. J. Mol. Biol. 90, 291-300. Abstract

Estes, S., and Lynch, M. (2003). Rapid fitness recovery in mutationally degraded lines of Caenorhabditis elegans. Evolution 57, 1022-1030. Abstract

Estes, S., Phillips, P.C., Denver, D.R., Thomas, W.K., and Lynch, M. (2004). Mutation accumulation in populations of varying size: the distribution of mutational effects for fitness correlates in Caenorhabditis elegans. Genetics 166, 1269-1279. Abstract Article

Fatt, H.V. (1967). Nutritional requirements for reproduction of a temperature-sensitive nematode, reared in axenic culture. Exp. Biol. Med. 124, 897-903. Abstract

Fatt, H.V., and Dougherty, E.C. (1963). Genetic control of differential heat tolerance in two strains of the nematode Caenorhabditis elegans. Science 141, 266-267. Abstract Article

Félix, M.-A. (2004). Alternative morphs and plasticity of vulval development in a rhabditid nematode species. Dev. Genes. Evol. 214, 55-63. Abstract Article

Félix, M.-A., Ashe, A., Piffaretti, J., Wu, G., Nuez, I., Bélicard, T., Jiang, Y., Zhao, G., Franz, C.J., Goldstein, L.D., et al. (2011). Natural and experimental infection of Caenorhabditis nematodes by novel viruses related to nodaviruses. PLoS Biol. 9, e1000586. Abstract Article

Félix, M.A., Braendle, C., and Cutter, A.D. (2014). A streamlined system for species diagnosis in Caenorhabditis (Nematoda: Rhabditidae) with name designations for 15 distinct biological species. PLoS One 9, e94723. Abstract Article

Ferris, H., and Hieb, W.F. (2015). Ellsworth C. Dougherty: a pioneer in the selection of Caenorhabditis elegans as a model organism. Genetics 200, 991-1002. Abstract Article

Finney, M., Ruvkun, G., and Horvitz, H.R. (1988). The C. elegans cell lineage and differentiation gene unc-86 encodes a protein containing a homeo domain and extended sequence similarity to mammalian transcription factors. Cell 55, 757-769. Abstract Article

Fodor, A., Riddle, D., Nemson, F.K., and Golden, J.W. (1983). Comparison of a new wild-type Caenorhabditis briggsae with laboratory strains of C. briggsae and C. elegans. Nematologica 29, 203-217. Article 
Gainutdinov, M.K., Timoshenko, A.K., Gindina, V.V., Gainutdinov, T.M., and Kalinnikova, T.B. (2007). Adaptation of Caenorhabditis elegans laboratory populations to high temperatures as a model of the evolution of thermotolerance in poikilothermal Metazoa. Dokl. Biol. Sci. 413, 149-152. Article

Gibert, M.-A., Starck, J., and Béguet, B. (1984). Role of the gonad cytoplasmic core during oogenesis of the nematode Caenorhabditis elegans. Biol. Cell 50, 77-86. Abstract Article

Gimond, C., Jovelin, R., Han, S., Ferrari, C., Cutter, A.D., and Braendle, C. (2013). Outbreeding depression with low genetic variation in selfing Caenorhabditis nematodes. Evolution 67, 3087-3101. Abstract Article

Goldschmidt, R. (1908). Das Nervensystem von Ascaris lumbricoides und megalocephala. Z. wiss. Zool. 90, 73-136.

Goldschmidt, R. (1909). Das Nervensystem von Ascaris lumbricoides und megalocephala. II. Z. wiss. Zool. 92, 306-357. Article

Goldstein, B. (1992). Induction of gut in Caenorhabditis elegans embryos. Nature 357, 255-257. Abstract Article

Goldstein, B., Frisse, L.M., and Thomas, W.K. (1998). Embryonic axis specification in nematodes: evolution of the first step in development. Curr. Biol. 8, 157-160. Abstract Article

Goldstein, B., and Hird, S. (1996). Specification of the antero-posterior axis in Caenorhabditis elegans. Development 122, 1467-1474. Abstract Article

Goldstein, P., and Curis, M. (1987). Age-related changes in the meiotic chromosomes of the nematode Caenorhabditis elegans. Mech. Ageing Dev. 40, 115-130. Abstract Article

Gönczy, P. (2012). Towards a molecular architecture of centriole assembly. Nat. Rev. Mol. Cell. Biol. 13, 425-435. Abstract Article

Gray, J.C., and Cutter, A.D. (2014). Mainstreaming Caenorhabditis elegans in experimental evolution. Proc. Biol. Sci. 281, 20133055. Abstract Article

Greenwald, I.S. (1985). lin-12, a nematode homeotic gene, is homologous to a set of mammalian proteins that includes epidermal growth factor. Cell 43, 583-590. Abstract Article

Grishok, A., Tabara, H., and Mello, C.C. (2000). Genetic requirements for inheritance of RNAi in C. elegans. Science 287, 2494-2497. Abstract Article

Gu, S.G., Pak, J., Guang, S., Maniar, J.M., Kennedy, S., and Fire, A. (2012). Amplification of siRNA in Caenorhabditis elegans generates a transgenerational sequence-targeted histone H3 lysine 9 methylation footprint. Nat. Genet. 44, 157-164. Abstract Article

Hamoir, G. (1992). The discovery of meiosis by E. Van Beneden: a breakthrough in the morphological phase of heredity. Int. J. Dev. Biol. 36, 9-15. Abstract Article

Hansen, E.L., and Cryan, W.S. (1966). Continuous axenic culture of free-living nematodes. Nematologica 12, 138-142.

Hansen, E.L., Yarwood, E.A., Nicholas, W.L., and Sayre, F.W. (1960). Differential nutritional requirements for reproduction of two strains of Caenorhabditis elegans in axenic culture. Nematologica 5, 27-31. Abstract

Harvey, S.C., Gemmill, A.W., Read, A.F., and Viney, M.E. (2000). The control of morph development in the parasitic nematode Strongyloides ratti. Proc. R. Soc. Lond. Ser. B 267, 2057-2063. Abstract Article

Harvey, S.C., and Viney, M.E. (2001). Sex determination in the parasitic nematode Strongyloides ratti. Genetics 158, 1527-1533. Abstract Article 
Hawley, R.S., and Gilliland, W.D. (2006). Sometimes the result is not the answer: the truths and the lies that come from using the complementation test. Genetics 174, 5-15. Abstract Article

Hechler, H.C. (1968). Postembryonic development and reproduction in Diploscapter coronata (Nematoda: Rhabditidae). Proc. Helm. Soc. Wash. 35, 24-30. Article

Hertwig, P. (1920). Abweichende Form der Parthenogenese bei einer Mutation von Rhabditis pellio. Arch. Mikrosk. Anat. 94, 303-337. Article

Hertwig, P. (1922). Beobachtungen über die Fortpflanzungsweise und die systematische Einteilung der Regenwurmnematoden. Z. wiss. Zool. 119, 539-558.

Hieb, W.F., and Dougherty, E.C. (1966). Evidence for tricarboxylic acid cycle in Panagrellus redivivus and changes observed under varying conditions of culture. Nematologica 12, 93.

Hieb, W.F., and Rothstein, M. (1968). Sterol requirement for reproduction of a free-living nematode. Science 160, 778-780. Abstract Article

Hieb, W.F., Stokstad, E.L., and Rothstein, M. (1970). Heme requirement for reproduction of a free-living nematode. Science 168, 143-144. Abstract Article

Hilbert, M., Erat, M.C., Hachet, V., Guichard, P., Blank, I.D., Fluckiger, I., Slater, L., Lowe, E.D., Hatzopoulos, G.N., Steinmetz, M.O., et al. (2013). Caenorhabditis elegans centriolar protein SAS-6 forms a spiral that is consistent with imparting a ninefold symmetry. Proc. Natl. Acad. Sci. U. S. A. 110, 11373-11378. Abstract Article

Hirschmann, H. (1951). Über das Vorkommen zweier Mundhöhlentypen bei Diplogaster lheritieri Maupas und Diplogaster biformis n. sp. und die Entstehung dieser hermaphroditischen Art aus Diplogaster lheritieri. Zool. Jahrb. 80, 132-170.

Hodgkin, J. (1985). Novel nematode amber suppressors. Genetics 111, 287-310. Abstract Article

Hodgkin, J. (1988). Sexual dimorphism and sex determination. In The nematode Caenorhabditis elegans, W.B. Wood, ed. (Cold Spring Harbor, NY: Cold Spring Harbor Laboratory Press). Article

Hodgkin, J. (2002a). Exploring the envelope. Systematic alteration in the sex-determination system of the nematode Caenorhabditis elegans. Genetics 162, 767-780. Abstract Article

Hodgkin, J. (2002b). One lucky XX male: isolation of the first Caenorhabditis elegans sex-determination mutants. Genetics 162, 1501-1504. Abstract Article

Hodgkin, J., and Brenner, S. (1977). Mutations causing transformation of sexual phenotype in the nematode Caenorhabditis elegans. Genetics 86, 275-287. Abstract Article

Hodgkin, J., and Doniach, T. (1997). Natural variation and copulatory plug formation in Caenorhabditis elegans. Genetics 146, 149-164. Abstract Article

Hodgkin, J., Horvitz, H.R., and Brenner, S. (1979). Nondisjunction mutants of the nematode Caenorhabditis elegans. Genetics 91, 67-94. Abstract Article

Honda, H. (1925). Experimental and cytological studies on bisexual and hermaphrodite free-living nematodes, with special reference to problems of sex. J. Morph. Physiol. 40, 191-233. Article

Horvitz, H.R., Brenner, S., Hodgkin, J., and Herman, R.K. (1979). A uniform genetic nomenclature for the nematode Caenorhabditis elegans. Mol. Gen. Genet. 175, 129-133. Abstract

Horvitz, H.R., and Sulston, J.E. (1980). Isolation and genetic characterization of cell-lineage mutants of the nematode Caenorhabditis elegans. Genetics 96, 435-454. Abstract Article 
Houthoofd, W., Willems, M., Jacobsen, K., Coomans, A., and Borgonie, G. (2008). The embryonic cell lineage of the nematode Rhabditophanes sp. Int. J. Dev. Biol. 52, 963-967. Abstract Article

$\mathrm{Hu}$, P.J. Dauer (August 08, 2007), WormBook, ed. The C. elegans Research Community, WormBook, doi/10.1895/wormbook.1.144.1, http://www.wormbook.org. Abstract Article

Isnenghi, E., Cassada, R., Smith, K., Denich, K., Radnia, K., and von Ehrenstein, G. (1983). Maternal effects and temperature-sensitive period of mutations affecting embryogenesis in Caenorhabditis elegans. Dev. Biol 98, 465-480. Abstract Article

Jacob, F. (1969). Recherche d'un organisme permettant l'analyse génétique de la différenciation cellulaire et de la morphogenèse. In Annuaire du Collège de France (Paris: Collège de France), pp. 195-196.

Jacob, F. (1970). Etude d'un organisme multicellulaire. In Annuaire du Collège de France (Paris: Collège de France), pp. 207-208.

Jacob, F. (1997). La souris, la mouche et l'homme (Paris, France: Odile Jacob).

Ji N. and van Oudenaarden A. Single molecule fluorescent in situ hybridization (smFISH) of C. elegans worms and embryos (December 13, 2012), WormBook, ed. The C. elegans Research Community, WormBook, doi/10.1895/wormbook.1.153.1, http://www.wormbook.org. Abstract Article

Jinks, J.L. (1964). Extrachromosomal inheritance (Englewood Cliffs, NJ: Prentice Hall).

Johnson, T.E., and Hutchinson, E.W. (1993). Absence of strong heterosis for lifespan and other life history traits in Caenorhabditis elegans. Genetics 134, 465-474. Abstract Article

Juang, B.T., Gu, C., Starnes, L., Palladino, F., Goga, A., Kennedy, S., and L'Etoile, N.D. (2013). Endogenous nuclear RNAi mediates behavioral adaptation to odor. Cell 154, 1010-1022. Abstract Article

Judson, H.F. (1996). The eighth day of creation (Cold Spring Harbor, NY: Cold Spring Harbor Laboratory Press).

Keightley, P.D., Davies, E.K., Peters, A.D., and Shaw, R.G. (2000). Properties of ethylmethane sulfonate-induced mutations affecting life-history traits in Caenorhabditis elegans and inferences about bivariate distributions of mutation effects. Genetics 156, 143-154. Abstract Article

Kimble, J., and Hirsh, D. (1979). Post-embryonic cell lineages of the hermaphrodite and male gonads in Caenorhabditis elegans. Dev. Biol. 70, 396-417. Abstract Article

Kiontke, K., Barrière, A., Kolotuev, I., Podbilewicz, B., Sommer, R.J., Fitch, D.H.A., and Félix, M.-A. (2007). Trends, stasis and drift in the evolution of nematode vulva development. Curr. Biol. 17, 1925-1937. Abstract Article

Kiontke, K., Félix, M.-A., Ailion, M., Rockman, M.V., Braendle, C., Pénigault, J.-B., and Fitch, D.H. (2011). A phylogeny and molecular barcodes for Caenorhabditis, with numerous new species from rotting fruits. BMC Evol. Biol. 11, 339. Abstract Article

Kirienko, N.V., Mani, K., and Fay, D.S. (2010). Cancer models in Caenorhabditis elegans. Dev. Dyn. 239, 1413-1448. Abstract Article

Klass, M.R. (1977). Aging in the nematode Ceanorhabditis elegans: major biological and environmental factors influencing life span. Mech. Aging Dev. 6, 413-429. Abstract

Kohler, R.E. (1994). Lords of the fly - Drosophila genetics and the experimental life (Chicago, IL: University of Chicago Press).

Korswagen, H.C., Durbin, R.M., Smits, M.T., and Plasterk, R.H.A. (1996). Transposon Tc1-derived, sequence-tagged sites in Caenorhabditis elegans as markers for gene mapping. Proc. Natl. Acad. Sci. U. S. A. 93, 14680-14685. Abstract Article 
Krüger, E. (1913). Fortpflanzung und Keimzellenbildung von Rhabditis aberrans, nov. sp. Z. wiss. Zool. 105, $87-124$.

Lahl, V., Sadler, B., and Schierenberg, E. (2006). Egg development in parthenogenetic nematodes: variations in meiosis and axis formation. Int. J. Dev. Biol. 50, 393-398. Abstract Article

Lansing, A.I. (1954). A non-genic factor in the longevity of rotifers. Ann. NY Acad. Sci. 57, 455-464. Abstract Article

Laufer, J.S., Bazzicalupo, P., and Wood, W.B. (1980). Segregation of developmental potential in early embryos of Caenorhabditis elegans. Cell 19, 569-577. Abstract Article

Laufer, J.S., and von Ehrenstein, G. (1981). Nematode development after removal of egg cytoplasm: Absence of localized unbound determinants. Science 211, 402-404. Abstract Article

Levitt, A., and Emmons, S.W. (1989). The Tc2 transposon in Caenorhabditis elegans. Proc. Natl. Acad. Sci. U. S. A. $86,3232-3236$. Abstract Article

Liao, L.W., Rosenzweig, B., and Hirsh, D. (1983). Analysis of a transposable element in Caenorhabditis elegans. Proc. Natl. Acad. Sci. U. S. A. 80, 3585-3589. Abstract Article

Lim, J.G., Stine, R.R., and Yanowitz, J.L. (2008). Domain-specific regulation of recombination in Caenorhabditis elegans in response to temperature, age and sex. Genetics 180, 715-726. Abstract Article

Lin, K.T., Broitman-Maduro, G., Hung, W.W., Cervantes, S., and Maduro, M.F. (2009). Knockdown of SKN-1 and the Wnt effector TCF/POP-1 reveals differences in endomesoderm specification in $C$. briggsae as compared with $C$. elegans. Dev. Biol. 325, 296-306. Abstract Article

Liu, Q.L., Thomas, V.P., and Williamson, V.M. (2007). Meiotic parthenogenesis in a root-knot nematode results in rapid genomic homozygosity. Genetics 176, 1483-1490. Abstract Article

Lower, W.R. (1966). Letter from William R. Lower to Sydney Brenner, S. Brenner, ed. (CSHL Archives Repository), Reference SB/1/1/377/3. http://libgallery.cshl.edu/items/show/62990

Lower, W.R., Hansen, E.L., and Yarwood, E.A. (1968). Selection for adaptation to increased temperatures in free-living nematodes. Life Sci. II 7, 139-146. Abstract Article

Lower, W.R., Willett, J.D., and Hansen, E.L. (1970). Selection for adaptation to increased temperatures in free-living nematodes - II. Some lipid differences in Panagrellus redivivus. Comp. Biochem. Physiol. 34, 473-479. Abstract

Lu, N.C., Cheng, A.C., and Briggs, G.M. (1983). A study of nutritional requirements in Caenorhabditis elegans. Nematologica 29, 425-434. Article

Lu, N.C., and Goetsch, K.M. (1993). Carbohydrate requirement of Caenorhabditis elegans and the final development of a chemically defined medium. Nematologica 39, 303-311. Article

Lu, N.C., Newton, C., and Stokstad, E.L.R. (1977). The requirement of sterol and various sterol precursors in free-living nematodes. Nematologica 23, 57-61. Article

Lui, D.Y., and Colaiacovo, M.P. (2013). Meiotic development in Caenorhabditis elegans. Adv. Exp. Med. Biol. 757, 133-170. Abstract

Luo, S., Kleemann, G.A., Ashraf, J.M., Shaw, W.M., and Murphy, C.T. (2010). TGF- $\beta$ and insulin signaling regulate reproductive aging via oocyte and germline quality maintenance. Cell 143, 299-312. Abstract Article

Maderspacher, F. (2008). Theodor Boveri and the natural experiment. Curr. Biol. 18, R279-R286. Abstract Article

Madl, J.E., and Herman, R.K. (1979). Polyploids and sex determination in Caenorhabditis elegans. Genetics 93, 393-402. Abstract Article 
Malone, C.J., Misner, L., Le Bot, N., Tsai, M.C., Campbell, J.M., Ahringer, J., and White, J.G. (2003). The C. elegans hook protein, ZYG-12, mediates the essential attachment between the centrosome and nucleus. Cell 115, 825-836. Abstract Article

Martini, E. (1923). Die Zellkonstanz und ihre Beziehungen zu anderen zoologischen Vorwürfen. Zeit. Anat. Entwick. 70, 179-250. Article

Maupas, E. (1899). La mue et l'enkystement chez les nématodes. Arch. Zool. Exp. Gen. 7, 563-628. Article

Maupas, E. (1900). Modes et formes de reproduction des nématodes. Arch. Zool. Exp. Gen. 8, 463-624.

Maupas, E. (1919). Essais d'hybridation chez des nématodes. Bull. Biol. Fr. Belg. 52, 466-498.

McGrath, P.T., Rockman, M.V., Zimmer, M., Jang, H., Macosko, E.Z., Kruglyak, L., and Bargmann, C.I. (2009). Quantitative mapping of a digenic behavioral trait implicates globin variation in C. elegans sensory behaviors. Neuron 61, 692-699. Abstract Article

McGrath, P.T., Xu, Y., Ailion, M., Garrison, J.L., Butcher, R.A., and Bargmann, C.I. (2011). Parallel evolution of domesticated Caenorhabditis species targets pheromone receptor genes. Nature 477, 321-325. Abstract Article

Mello, C., and Fire, A. (1995). DNA transformation. In Caenorhabditis elegans Modern Biological Analysis of an Organism, H.F. Epstein, and D.C. Shakes, eds. (San Diego: Academic Press), pp. 451-482.

Melters, D.P., Paliulis, L.V., Korf, I.F., and Chan, S.W. (2012). Holocentric chromosomes: convergent evolution, meiotic adaptations, and genomic analysis. Chromosome Res. 20, 579-593. Abstract Article

Meneely, P.M. (1994). Sex determination in polyploids of Caenorhabditis elegans. Genetics 137, 467-481. Abstract Article

Meneely, P.M., Farago, A.F., and Kauffman, T.M. (2002). Crossover distribution and high interference for both the $\mathrm{X}$ chromosome and an autosome during oogenesis and spermatogenesis in Caenorhabditis elegans. Genetics 162, 1169-1177. Abstract Article

Metcalf, H. (1903). Cultural studies of a nematode associated with plant decay. Trans. Amer. Microscop. Soc. 24, 89-102.

Meyer, B. J. X-Chromosome dosage compensation (June 25, 2005), WormBook, ed. The C. elegans Research Community, WormBook, doi/10.1895/wormbook.1.8.1, http://www.wormbook.org. Abstract Article

Miwa, J., Schierenberg, E., Miwa, S., and von Ehrenstein, G. (1980). Genetics and mode of expression of temperature-sensitive mutations arresting embryonic development in Caenorhabditis elegans. Dev. Biol. 76, 160-174. Abstract Article

Moerman, D.G., Benian, G.M., and Waterston, R.H. (1986). Molecular cloning of the muscle gene unc-22 in Caenorhabditis elegans by Tc1 transposon tagging. Proc. Natl. Acad. Sci. U. S. A. 83, 2579-2583. Abstract Article

Moerman, D.G., and Waterston, R.H. (1984). Spontaneous unstable unc-22 IV mutations in Caenorhabditis elegans var. Bergerac. Genetics 108, 859-877. Abstract Article

Mori, I., Moerman, D.G., and Waterston, R.H. (1988). Analysis of a mutator activity necessary for germline transposition and excision of Tc1 transposable elements in Caenorhabditis elegans. Genetics 120, 397-407. Abstract Article

Morran, L.T., Parmenter, M.D., and Phillips, P\.C. (2009). Mutation load and rapid adaptation favour outcrossing over self-fertilization. Nature 462, 350-352. Abstract Article

Mounier, N., and Brun, J.-L. (1980). A cytogenetical analysis of sterile mutants in Caenorhabditis elegans. Can. J. Genet. Cytol. 22, 391-403. Abstract

Müller, F., Bernard, V., and Tobler, H. (1996). Chromatin diminution in nematodes. Bioessays 18, $133-138$. Abstract Article 
Muller, H. (1903). Beitrag zur Embryonalentwickelung der Ascaris megalocephala. Zoologica 17, 1-30.

Nabeshima, K., Mlynarczyk-Evans, S., and Villeneuve, A.M. (2011). Chromosome painting reveals asynaptic full alignment of homologs and HIM-8-dependent remodeling of $\mathrm{X}$ chromosome territories during Caenorhabditis elegans meiosis. PLoS Genet. 7, e1002231. Abstract Article

Nabeshima, K., Villeneuve, A.M., and Colaiacovo, M.P. (2005). Crossing over is coupled to late meiotic prophase bivalent differentiation through asymmetric disassembly of the SC. J. Cell Biol. 168, 683-689. Abstract Article

Nemetschke, L., Eberhardt, A.G., Hertzberg, H., and Streit, A. (2010a). Genetics, chromatin diminution and sex chromosome evolution in the parasitic nematode genus Strongyloides. Curr. Biol. 20, 1687-1696. Abstract Article

Nemetschke, L., Eberhardt, A.G., Viney, M.E., and Streit, A. (2010b). A genetic map of the animal-parasitic nematode Strongyloides ratti. Mol. Biochem. Parasitol. 169, 124-127. Abstract Article

Nicholas, W.L. (1975). The biology of free-living nematodes (London: Oxford University Press). Abstract

Nicholas, W.L., Dougherty, E.C., and Hansen, E.L. (1959). Axenic cultivation of Caenorhabditis briggsae (Nematoda: Rhabditidae) with chemically undefined supplements; comparative studies with related nematodes. Ann. NY Acad. Sci. 411, 218-236. Article

Nigon, V. (1943). Le déterminisme du sexe chez un nématode libre hermaphrodite (Rhabditis elegans Maupas). C. R. Soc. Biol. 137, 40-41.

Nigon, V. (1946). Le determinisme du sexe chez un Nematode libre hermaphrodite: Rhabditis dolichura Schneider. Bull. Soc. Zool. France 71, 78-86.

Nigon, V. (1947). Le déterminisme du sexe et la pseudogamie chez un Nématode parthénogénétique, Rhabditis monohystera Bütschli. Bull. Biol. Fr. Belg. 81, 33-37.

Nigon, V. (1949a). Modalités de la reproduction et déterminisme du sexe chez quelques nématodes libres. Ann. des Sc. Nat., Zool. 11, 1-132.

Nigon, V. (1949b). Effets de la polyploïdie chez un nématode libre. C. R. Acad. Sc. Paris D 228, 1161-1162.

Nigon, V. (1951a). Polyploïdie expérimentale chez un nématode libre, Rhabditis elegans Maupas. Bull. Biol. Fr. Belg. 85, 187-225.

Nigon, V. (1951b). Effets d'une élévation de température sur la prophase méiotique d'un Nématode libre. C. R. Acad. Sc. Paris D 232, 1032-1034.

Nigon, V. (1952a). Modifications expérimentales de la proportion des sexes chez un Nématode pseudogame. C. R. Acad. Sc. Paris D 234, 2568-2570.

Nigon, V. (1954). Contributions à la critique expérimentale des théories de la continuité germinale et de la lignée pure. Acta Biol. Acad. Sci. Hungar. 5, 96-117.

Nigon, V. (1965). Développement et reproduction des nématodes. In Traité de Zoologie, P.-P. Grassé, ed. (Paris: Masson).

Nigon, V. (1966/1970). Nigon, V., S. Brenner, ed. (CSHL Archives Repository), Reference SB/1/1/457. http://libgallery.cshl.edu/items/show/63774

Nigon, V., and Brun, J. (1955). L'évolution des structures nucléaires dans l'ovogenèse de Caenorhabditis elegans Maupas 1900. Chromosoma 7, 129-169. Abstract

Nigon, V., and Brun, J. (1967). Génétique et évolution des Nématodes libres. Perspectives tirées de l'étude de Caenorhabditis elegans. Experientia 23, 161-240. Abstract 
Nigon, V., and Dougherty, E.C. (1949). Reproductive patterns and attempts at reciprocal crossing of Rhabditis elegans Maupas, 1900, and Rhabditis briggsae Dougherty and Nigon, 1949 (Nematoda: Rhabditidae). J. Exp. Zool. 112, 485-503. Abstract

Nigon, V., and Dougherty, E.C. (1950). A dwarf mutation in a nematode; a morphological mutant of Rhabditis briggsae, a free-living soil nematode. J. Hered. 41, 103-109. Abstract Article

Nigon, V., and Dougherty, E.C. (1953). Variations dans la vitesse de croissance chez un Nématode Caenorhabditis elegans (Maupas). Caryologia, Suppl.: Atti Del IX Congresso Internazionale Di Genetica 6, 796-797.

Nigon, V., Guerrier, P., and Monin, H. (1960). L'architecture polaire de l'œuf et les mouvements des constituants cellulaires au cours des premières étapes du développement chez quelques Nématodes. Bull. biol. Fr. Belg. 93, 131-202.

Nigon, V., and Roman, E. (1952). Le déterminisme du sexe et le développement cyclique de Strongyloides ratti. Bull. Biol. Fr. Belg. 86, 405-448.

Nirenberg, M.W., and Tabery, J. (2001). Interview with Marshall W. Nirenberg. In Profiles in Science - The Marshall W. Nirenberg papers, National Library of Medicine, ed. http://profiles.nlm.nih.gov/ps/retrieve/Resource Metadata/JJBBXD

Nonnenmacher-Godet, J., and Dougherty, E.C. (1964). Incorporation of tritiated thymidine in the cells of Caenorhabditis briggsae (Nematoda) reared in axenic culture. J. Cell Biol. 22, 281-290. Abstract Article

Oegema, K. and Hyman, A.A. Cell division (January 19, 2006), WormBook, ed. The C. elegans Research Community, WormBook, doi/10.1895/wormbook.1.72.1,http://www.wormbook.org. Abstract Article

Osche, G. (1952). Systematik und Phylogenie der Gattung Rhabditis (Nematoda). Zoologische Jahrbücher (Systematik) 81, 190-280.

Osche, G. (1954). Über die gegenwärtig ablaufende Entstehung von Zwillings- und Komplementärarten bei Rhabditiden (Nematodes). (Fötalisation und Artbildung). Zool. Jahrb. (Syst.) 82, 618-654.

Ouazana, R. (1975). Etude génétique physiologique et morphologique du nanisme dumpy chez le nématode hermaphrodite Caenorhabditis elegans variété Bergerac (Thèse, Université Claude-Bernard Lyon I).

Ouazana, R. (1978). A morphological temperature-sensitive mutant of the nematode C. elegans var. Bergerac. Experientia 34, 170-171.

Ouazana, R., and Brun, J. (1978). Effect of dumpiness on the development and functioning of eutelic and non-eutelic organs in the nematode Caenorhabditis elegans. Genetica 49, 45-52. Article

Ouazana, R., and Brun, J.-L. (1975). Recombinaison intracistronique au niveau d'un locus du nanisme chez le Nématode hermaphrodite Caenorhabditis elegans. C. R. Acad. Sc. Paris D 280, 1895-1898.

Ouazana, R., Garrone, R., and Godet, J. (1985). Characterization of morphological and biochemical defects in the cuticle of a dumpy mutant of Caenorhabditis elegans. Comp. Biochem. Physiol. 80B, 481-484. Article

Ouazana, R., and Gibert, M.-A. (1979). Cuticular collagen composition of the nematode C. elegans, Bergerac wild-type strain. C. R. Acad. Sci. Paris D 288, 911-914.

Ouazana, R., and Herbage, D. (1981). Biochemical characterization of the cuticle collagen of the nematode Caenorhabditis elegans. Biochem. Biophys. Acta 669, 236-243. Abstract Article

Pai, S. (1928). Die Phasen des Lebenscyclus der Anguillula aceti Ehrbg. und ihre experimentell-morphologische Beeinflussung. Z. wiss. Zool. 131, 293-344.

Pal, C., and Miklos, I. (1999). Epigenetic inheritance, genetic assimilation and speciation. J. Theor. Biol. 200, 19-37. Abstract Article 
Penkner, A., Tang, L., Novatchkova, M., Ladurner, M., Fridkin, A., Gruenbaum, Y., Schweizer, D., Loidl, J., and Jantsch, V. (2007). The nuclear envelope protein Matefin/SUN-1 is required for homologous pairing in $C$. elegans meiosis. Dev. Cell 12, 873-885. Abstract Article

Penkner, A.M., Fridkin, A., Gloggnitzer, J., Baudrimont, A., Machacek, T., Woglar, A., Csaszar, E., Pasierbek, P., Ammerer, G., Gruenbaum, Y., et al. (2009). Meiotic chromosome homology search involves modifications of the nuclear envelope protein Matefin/SUN-1. Cell 139, 920-933. Abstract Article

Perelman, D., and Lu, N.C. (2000). Requirements for branched amino acids and their interactions in the nematode Caenorhabditis elegans. Nematology 2, 501-506. Article

Phillips, C.M., and Dernburg, A.F. (2006). A family of zinc-finger proteins is required for chromosome-specific pairing and synapsis during meiosis in C. elegans. Dev. Cell 11,817-829. Abstract Article

Phillips, C.M., Wong, C., Bhalla, N., Carlton, P.M., Weiser, P., Meneely, P.M., and Dernburg, A.F. (2005). HIM-8 binds to the $\mathrm{X}$ chromosome pairing center and mediates chromosome-specific meiotic synapsis. Cell 123, 1051-1063. Abstract Article

Pires-daSilva, A. (2007). Evolution of the control of sexual identity in nematodes. Semin. Cell Dev. Biol. 18, 362-370. Abstract Article

Plasterk, R.H., and van Luenen, H.G.A.M. (1997). Transposons. In C. elegans II, D. Riddle, T. Blumenthal, B.J. Meyer, and J.R. Priess, eds. (Cold Spring Harbor, NY: Cold Spring Harbor Laboratory Press), pp. 97-116. Article

Potts, F.A. (1910). Notes on the free-living nematodes. Q. J. Microscop. Sci. 55, 433-485. Article

Priess, J.R., and Thomson, J.N. (1987). Cellular interactions in early Caenorhabditis elegans embryos. Cell 48, 241-250. Abstract Article

Ragsdale, E.J., Muller, M.R., Rodelsperger, C., and Sommer, R.J. (2013). A developmental switch coupled to the evolution of plasticity acts through a sulfatase. Cell 155, 922-933. Abstract Article

Rao, A.U., Carta, L.K., Lesuisse, E., and Hamza, I. (2005). Lack of heme synthesis in a free-living eukaryote. Proc. Natl. Acad. Sci. U. S. A. 102, 4270-4275. Abstract Article

Rechavi, O., Houri-Ze'evi, L., Anava, S., Goh, W.S., Kerk, S.Y., Hannon, G.J., and Hobert, O. (2014). Starvation-induced transgenerational inheritance of small RNAs in C. elegans. Cell 158, 277-287. Abstract Article

Remy, J.J. (2010). Stable inheritance of an acquired behavior in Caenorhabditis elegans. Curr. Biol. 20, R877-878. Abstract Article

Riddle, D.L., Blumenthal, T., Meyer, B.J., and Priess, J.R. (1997). Introduction to C. elegans. In C. elegans II, D.L. Riddle, T. Blumenthal, B.J. Meyer, and J.R. Priess, eds. (Cold Spring Harbor, NY: Cold Spring Harbor Laboratory Press), pp. 1-22. Article

Rog, O., and Dernburg, A.F. (2013). Chromosome pairing and synapsis during Caenorhabditis elegans meiosis. Curr. Opin. Cell Biol. 25, 349-356. Abstract Article

Rog, O., and Dernburg, A.F. (2015). Direct visualization reveals kinetics of meiotic chromosome synapsis. Cell Rep. 10, 1639-1645. Abstract Article

Rose, A.M., and Baillie, D.L. (1979). The effect of temperature and parental age on recombination and nondisjunction in Caenorhabditis elegans. Genetics 92, 409-418. Abstract Article

Rose L. and Gönczy P. Polarity establishment, asymmetric division and segregation of fate determinants in early $C$. elegans embryos (December 30, 2014), WormBook, ed. The C. elegans Research Community, WormBook, doi/10.1895/wormbook.1.30.2, http://www.wormbook.org. Abstract Article 
Ruvkun, G., Ambros, V., Coulson, A., Waterston, R., Sulston, J., and Horvitz, H.R. (1989). Molecular genetics of the Caenorhabditis elegans heterochronic gene lin-14. Genetics 121, 501-516. Abstract Article

Sato, A., Isaac, B., Phillips, C.M., Rillo, R., Carlton, P.M., Wynne, D.J., Kasad, R.A., and Dernburg, A.F. (2009). Cytoskeletal forces span the nuclear envelope to coordinate meiotic chromosome pairing and synapsis. Cell 139, 907-919. Abstract Article

Schedl, T., and Kimble, J. (1988). fog-2, a germ-line-specific sex determination gene required for hermaphrodite spermatogenesis in Caenorhabditis elegans. Genetics 119, 43-61. Abstract Article

Schierenberg, E. Embryological variation during nematode development (January 02, 2006), WormBook, ed. The C. elegans Research Community, WormBook, doi/10.1895/wormbook.1.55.1, http://www.wormbook.org. Abstract Article

Schierenberg, E., Miwa, J., and von Ehrenstein, G. (1980). Cell lineages and developmental defects of temperature-sensitive embryonic arrest mutants in Caenorhabditis elegans. Dev. Biol. 76 141-159. Abstract Article

Schiffer, P.H., Nsah, N.A., Grotehusmann, H., Kroiher, M., Loer, C., and Schierenberg, E. (2014). Developmental variations among Panagrolaimid nematodes indicate developmental system drift within a small taxonomic unit. Dev. Genes Evol. 224, 183-188. Abstract Article

Schleip, W. (1911). Das Verhalten des Chromatins bei Angiostomum (Rhabdonema) nigrovenosum. Ein Beitrag zur Kenntnis der Beziehungen zwischen Chromatin und Geschlechtsbestimmung. Arch. Zellf. 7, 87-138.

Schnabel, R. (1997). Why does a nematode have an invariant cell lineage? Semin. Cell Dev. Biol. 8, $341-349$. Abstract Article

Schnabel, R., and Priess, J. (1997). Specification of cell fates in the early embryo. In C. elegans II, D.L. Riddle, T. Blumenthal, B.J. Meyer, and J.R. Priess, eds. (Cold Spring Harbor, NY: Cold Spring Harbor Laboratory Press), pp. 361-382. Article

Schneider, A.F. (1866). Monographie der Nematoden (Berlin: G. Reimer). Article

Schott, D., Yanai, I., and Hunter, C.P. (2014). Natural RNA interference directs a heritable response to the environment. Sci. Rep. 4, 7387. Abstract Article

Schulte, R.D., Makus, C., Hasert, B., Michiels, N.K., and Schulenburg, H. (2010). Multiple reciprocal adaptations and rapid genetic change upon experimental coevolution of an animal host and its microbial parasite. Proc. Natl. Acad. Sci. U. S. A. 107, 7359-7364. Abstract Article

Schulze, J., and Schierenberg, E. (2011). Evolution of embryonic development in nematodes. EvoDevo 2 , 18. Abstract Article

Schvarzstein, M., Wignall, S.M., and Villeneuve, A.M. (2010). Coordinating cohesion, co-orientation, and congression during meiosis: lessons from holocentric chromosomes. Genes Dev. 24, 219-228. Abstract Article

Seidel, H.S., Ailion, M., Li, J., van Oudenaarden, A., Rockman, M.V., and Kruglyak, L. (2011). A novel sperm-delivered toxin causes late-stage embryo lethality and transmission ratio distortion in C. elegans. PLoS Biol. 9, e1001115. Abstract Article

Shakes, D.C., Neva, B.J., Huynh, H., Chaudhuri, J., and Pires-Dasilva, A. (2011). Asymmetric spermatocyte division as a mechanism for controlling sex ratios. Nat. Commun. 2, 157. Abstract Article

Shakes, D.C., Wu, J.C., Sadler, P.L., Laprade, K., Moore, L.L., Noritake, A., and Chu, D.S. (2009). Spermatogenesis-specific features of the meiotic program in Caenorhabditis elegans. PLoS Genet. 5, e1000611. Abstract Article

Shirayama, M., Seth, M., Lee, H.C., Gu, W., Ishidate, T., Conte, D., Jr., and Mello, C.C. (2012). piRNAs initiate an epigenetic memory of nonself RNA in the C. elegans germline. Cell 150, 65-77. Abstract Article 
Shook, D.R., Brooks, A., and Johnson, T.E. (1996). Mapping quantitative trait loci affecting life history traits in the nematode Caenorhabditis elegans. Genetics 142, 801-817. Abstract Article

Sikkink, K.L., Reynolds, R.M., Ituarte, C.M., Cresko, W.A., and Phillips, P.C. (2014). Rapid evolution of phenotypic plasticity and shifting thresholds of genetic assimilation in the nematode Caenorhabditis remanei. G3 4 , 1103-1112. Abstract Article

Simon, M., Sarkies, P., Ikegami, K., Doebley, A.-L., Goldstein, L.D., Mitchell, J., Sakaguchi, A., Miska, E.A., and Ahmed, S. (2014). Reduced insulin/IGF-1 signaling restores germ cell immortality to Caenorhabditis elegans Piwi mutants. Cell Rep. 7, 762-773. Abstract Article

Smelick, C., and Ahmed, S. (2005). Achieving immortality in the C. elegans germline. Ageing Res. Rev. 4, 67-82. Abstract Article

Sommer, R.J. Evolution of development in nematodes related to C. elegans (December 14, 2005), WormBook, ed. The C. elegans Research Community, WormBook, doi/10.1895/wormbook.1.46.1, http://www.wormbook.org. Abstract Article

Spemann, H. (1895). Zur Entwicklung des Strongylus paradoxus. Zool. Jahrb. Abt. Morphol. 8, 301-317.

Spieler, M., and Schierenberg, E. (1995). On the development of the alternating free-living and parasitic generations of the nematode Rhabdias bufoni. Invertebr. Repr. Dev. 28, 193-203.

Starck, J. (1984). Synthesis of oogenesis specific proteins in Caenorhabditis elegans: an approach to the study of vitellogenesis in a nematode. Int. J. Inv. Reprod. Dev. 7, 149-160. Article

Starck, J., Gibert, M.-A., Brun, J., and Bosch, C. (1983). Ribosomal RNA synthesis and processing during oogenesis of the free living nematode Caenorhabditis elegans. Comp. Biochem. Physiol. 75, 575-580. Article

Stent, G.S. (1965). Postcard from Lise, Francois, Inga, and Gunther to Sydney Brenner, S. Brenner, ed. (CSHL Archives Repository), pp. Reference SB/1/1/568/553. http://libgallery.cshl.edu/items/show/64760

Sterken, M.G., Snoek, L.B., Bosman, K.J., Daamen, J., Riksen, J.A., Bakker, J., Pijlman, G.P., and Kammenga, J.E. (2014). A heritable antiviral RNAi response limits Orsay virus infection in Caenorhabditis elegans N2. PLoS One 9, e89760. Abstract Article

Sterken, M.G., Snoek, L.B., Kammenga, J.E., and Andersen, E.C. (2015). The laboratory domestication of Caenorhabditis elegans. Trends Genet. 31, 224-231. Abstract Article

Sternberg, P.W., Vulval development (June, 25 2005), WormBook, ed. The C. elegans Research Community, WormBook, doi/10.1895/wormbook.1.6.1, http://www.wormbook.org. Abstract Article

Sternberg, P.W., and Horvitz, H.R. (1981). Gonadal cell lineages of the nematode Panagrellus redivivus and implications for evolution by the modification of cell lineage. Dev. Biol. 88, 147-166. Abstract Article

Sternberg, P.W., and Horvitz, H.R. (1982). Postembryonic nongonadal cell lineages of the nematode Panagrellus redivivus: Description and comparison with those of Caenorhabditis elegans. Dev. Biol. 93, 181-205. Abstract Article

Stiernagle, T. (2006). Maintenance of C. elegans. In WormBook, The C. elegans Research Community, ed., http://www.wormbook.org, doi/10.1895/wormbook.1.101.1 Abstract Article

Streit, A. (2008). Reproduction in Strongyloides (Nematoda): a life between sex and parthenogenesis. Parasitology 135, 285-294. Abstract Article

Sudhaus, W. (1974). Zur Systematik, Verbreitung, Ökologie und Biologie neuer und wenig bekannter Rhabditiden (Nematoda). 2. Teil. Zool. Jahrb. (Syst.) 101, 417-465. 
Sudhaus, W. (2011). Phylogenetic systematisation and catalogue of paraphyletic "Rhabditidae" (Secernentea, Nematoda). J. Nem. Morph. Syst. 14, 113-178. Article

Sudhaus, W., and Fürst von Lieven, A. (2003). A phylogenetic classification and catalogue of the Diplogastridae (Secernentea, Nematoda). J. Nem. Morph. Syst. 6, 43-90. Article

Sudhaus, W., and Kiontke, K. (2007). Comparison of the cryptic nematode species Caenorhabditis brenneri sp. n. and $C$. remanei (Nematoda: Rhabditidae) with the stem species pattern of the Caenorhabditis elegans group. Zootaxa 1456, 45-62. Article

Sulston, J., and Ferry, G. (2002). The Common Thread: A Story of Science, Politics, Ethics and the Human Genome (Washinton, DC: The Joseph Henry Press).

Sulston, J., and Horvitz, H.R. (1977). Postembryonic cell lineages of the nematode, Caenorhabditis elegans. Dev. Biol. 56, 110-156. Article

Sulston, J.E., and Brenner, S. (1974). The DNA of Caenorhabditis elegans. Genetics 77, 95-104. Abstract Article

Sulston, J.E., Schierenberg, E., White, J.G., and Thomson, J.N. (1983). The embryonic cell lineage of the nematode Caenorhabditis elegans. Dev. Biol. 100, 64-119. Abstract Article

Sulston, J.E., and White, J.G. (1980). Regulation and cell autonomy during postembryonic development of Caenorhabditis elegans. Dev. Biol. 78, 577-597. Abstract Article

Szewczyk, N.J., Kozak, E., and Conley, C.A. (2003). Chemically defined medium and Caenorhabditis elegans. BMC Biotechnol. 3, 19. Abstract Article

Szewczyk, N.J., Mancinelli, R.L., McLamb, W., Reed, D., Blumberg, B.S., and Conley, C.A. (2005). Caenorhabditis elegans survives atmospheric breakup of STS-107, Space Shuttle Columbia. Astrobiology 5, 690-705. Abstract Article

Tal, O., Kisdi, E., and Jablonka, E. (2010). Epigenetic contribution to covariance between relatives. Genetics 184 , 1037-1050. Abstract Article

The C. elegans Sequencing Consortium (1998). Genome sequence of the nematode C. elegans: a platform for investigating biology. Science 282, 2012-2018. Abstract Article

Theologidis, I., Chelo, I.M., Goy, C., and Teotonio, H. (2014). Reproductive assurance drives transitions to self-fertilization in experimental Caenorhabditis elegans. BMC Biol. 12, 93. Abstract Article

Thomas, C.G., Woodruff, G.C., and Haag, E.S. (2012). Causes and consequences of the evolution of reproductive mode in Caenorhabditis nematodes. Trends Genet. 28, 213-220. Abstract Article

Trent, C., Tsung, N., and Horvitz, H.R. (1983). Egg-laying defective mutants of the nematode Caenorhabditis elegans. Genetics 104, 619-647. Abstract Article

Triantaphyllou, A.C., and Hirschmann, H. (1964). Reproduction in plant and soil nematodes. Annu. Rev. Phytopathol. 2, 57-80. Article

Vader, G., and Musacchio, A. (2014). HORMA domains at the heart of meiotic chromosome dynamics. Dev. Cell 31, 389-391. Abstract Article

Van Beneden, E. (1883). Recherches sur la fécondation et maturation de l'œuf, la fécondation et la division cellulaire. Arch. Biol. 4, 265-640. Article

van Leeuwenhoeck, A. (1676). Extract of a letter written to the publisher by Mr. Leewenhoeck from Delft, April 21, 1676: Concerning the texture of trees, and some remarkable discovery in wine; together with some notes thereon. Phil. Trans. 11, 653-660. 
Vanfleteren, J.R. (1974). Nematode growth factor. Nature 248, 255-257. Abstract

Vassilieva, L.L., Hook, A.M., and Lynch, M. (2000). The fitness effects of spontaneous mutations in Caenorhabditis elegans. Evolution 54, 1234-1246. Abstract Article

Vella, M.C. and Slack, F.J. C. elegans microRNAs (September 21, 2005), WormBook, ed. The C. elegans Research Community, WormBook, doi/10.1895/wormbook.1.26.1,http://www.wormbook.org. Abstract Article

Viney, M. (1996). Developmental switching in the parasitic nematode Strongyloides ratti. Proc. R. Soc. Lond. B 263, 201-208. Abstract Article

Viney, M.E. (1994). A genetic analysis of reproduction in Strongyloides ratti. Parasitology 109, 511-515. Abstract Article

Viney M.E., Lok J.B. The biology of Strongyloides spp. (July 16, 2015), WormBook, ed. The C. elegans Research Community, WormBook, doi/10.1895/wormbook.1.141.2, http://www.wormbook.org. Abstract Article

Viney, M.E., Matthews, B.E., and Walliker, D. (1992). On the biological and biochemical nature of cloned populations of Strongyloides ratti. J. Helminthol. 66, 45-52. Abstract Article

Viney, M.E., Matthews, B.E., and Walliker, D. (1993). Mating in the nematode parasite Strongyloides ratti: proof of genetic exchange. Proc. Biol. Sci. 254, 213-219. Abstract Article

Wertheim, B., Beukeboom, L.W., and van de Zande, L. (2013). Polyploidy in animals: effects of gene expression on sex determination, evolution and ecology. Cytogenet. Genome Res. 140, 256-269. Abstract Article

White, J.G., Southgate, E., Thomson, J.N., and Brenner, S. (1986). The structure of the nervous system of the nematode Caenorhabditis elegans. Phil. Trans. Roy. Soc., Lond. B. 314, 1-340. Abstract Article

Williams, B.D., Schrank, B., Huynh, C., Shownkeen, R., and Waterston, R.H. (1992). A genetic mapping system in Caenorhabditis elegans based on polymorphic sequence-tagged sites. Genetics 131, 609-624. Abstract Article

Wood, W.B. (1988). The nematode Caenorhabditis elegans (Cold Spring Harbor, NY: Cold Spring Harbor Laboratory Press).

Wood, W.B. (1991). Evidence from reversal of handedness in C. elegans for early cell interactions determining cell fates. Nature 349, 536-538. Abstract Article

Wood, W.B., Hecht, R., Carr, S., Vanderslice, R., Wolf, N., and Hirsh, D. (1980). Parental effects and phenotypic characterization of mutations that affect early development in Caenorhabditis elegans. Dev. Biol. 74, 446-469. Abstract Article

Woodruff, G.C., Eke, O., Baird, S.E., Félix, M.-A., and Haag, E.S. (2010). Insights into species divergence and the evolution of hermaphroditism from fertile interspecies hybrids of Caenorhabditis nematodes. Genetics 186, 997-1012. Abstract Article

Xiong, X., and Lu, N.C. (2011). Optimal levels of the essential amino acids histidine, lysine and threonine in Caenorhabditis elegans maintenance medium. Nematology 10, 539-544. Article

Yuan, J., Finney, M., Tsung, N., and Horvitz, H.R. (1991). Tc4, a Caenorhabditis elegans transposable element with an unusual foldback structure. Proc. Natl. Acad. Sci. U. S. A. 88, 3334-3338. Abstract Article

Zarkower, D. Somatic sex determination (February 10, 2006), WormBook, ed. The C. elegans Research Community, WormBook, doi/10.1895/wormbook.1.84.1, http://www.wormbook.org. Abstract Article

Zhao, Y., and Lu, N.C. (2008). Requirements of non-haem iron and haem iron in the nematode Caenorhabditis elegans. Nematology 13, 853-858. Article 
Zhou, K., Rolls, M.M., Hall, D.H., Malone, C.J., and Hanna-Rose, W. (2009). A ZYG-12-dynein interaction at the nuclear envelope defines cytoskeletal architecture in the C. elegans gonad. J. Cell Biol. 186, 229-241. Abstract Article

zur Strassen, O. (1896). Embryonalentwickelung der Ascaris megalocephala. Arch. Entw. Mech. Org. 3, $27-105$.

zur Strassen, O. (1959). Neue Beiträge zur Entwicklungsmechanik der Nematoden. Zoologica 107, 1-142.

\section{Appendix 1. Table 3. Main species mentioned in the text with their modern names and mode of reproduction.}

Table 3. Main species mentioned in the text with their modern names and mode of reproduction.

\begin{tabular}{|c|c|c|c|c|c|}
\hline $\begin{array}{l}\text { Species name } \\
\text { (old) }\end{array}$ & $\begin{array}{l}\text { Species name } \\
\text { (present) }\end{array}$ & Family & Reproduction & Male frequency & Authors \\
\hline $\begin{array}{l}\text { Alaimus } \\
\text { thamugadi } \\
\text { Maupas }\end{array}$ & & Alaimidae & $\mathrm{P}$ & Absent & Maupas, 1900 \\
\hline $\begin{array}{l}\text { Aphelenchus } \\
\text { agricola De } \\
\text { Man }\end{array}$ & $\begin{array}{l}\text { Aphelenchus } \\
\text { avenae }\end{array}$ & Aphelen-choididae & $\mathrm{P}$ & Absent & Maupas, 1900 \\
\hline $\begin{array}{l}\text { Cephalobus } \\
\text { dubius Maupas }\end{array}$ & & Cephalobidae & $\mathrm{P}$ & Absent & Maupas, 1900 \\
\hline $\begin{array}{l}\text { Cephalobus } \\
\text { lentus Maupas }\end{array}$ & Chiloplacus lentus & Cephalobidae & $\mathrm{P}$ & Absent & Maupas, 1900 \\
\hline $\begin{array}{l}\text { Diplogaster } \\
\text { linstowi Potts }\end{array}$ & $\begin{array}{l}\text { Pristionchus } \\
\text { linstowi }\end{array}$ & Diplogastridae & $\mathrm{H}$ & & Potts, 1910 \\
\hline $\begin{array}{l}\text { Diplogaster } \\
\text { maupasi } \text { Potts }\end{array}$ & $\begin{array}{l}\text { Pristionchus } \\
\text { maupasi }\end{array}$ & Diplogastridae & $\mathrm{H}$ & $\begin{array}{l}\text { (Up to } 30 \% \text { in } \\
\text { culture with } \\
\text { males) }\end{array}$ & Potts, 1910 \\
\hline $\begin{array}{l}\text { Diplogaster } \\
\text { minor Maupas }\end{array}$ & $\begin{array}{l}\text { Pristionchus } \\
\text { gallicus }\end{array}$ & Diplogastridae & $\mathrm{H}$ & Absent & Maupas, 1900 \\
\hline $\begin{array}{l}\text { Diplogaster } \\
\text { robustus Maupas }\end{array}$ & $\begin{array}{l}\text { Pristionchus } \\
\text { robustus }\end{array}$ & Diplogastridae & $\mathrm{H}$ & $0.13 / 1000$ & Maupas, 1900 \\
\hline $\begin{array}{l}\text { Macrolaimus } \\
\text { crucis Maupas }\end{array}$ & & Chamber-siellidae & $\mathrm{P}$ & Absent & Maupas, 1900 \\
\hline $\begin{array}{l}\text { Panagrolaimus } \\
\text { rigidus }\end{array}$ & $\begin{array}{l}\text { Panagrolaimus } \\
\text { rigidus }\end{array}$ & Panagrolai-midae & G & & Nigon, 1949a \\
\hline $\begin{array}{l}\text { Plectus cirratus } \\
\text { Bastian }\end{array}$ & & Plectidae & $\mathrm{P}$ & Absent & Maupas, 1900 \\
\hline $\begin{array}{l}\text { Rhabditis } \\
\text { viguieri Maupas }\end{array}$ & Reiterina viguieri & Rhabditidae & $\mathrm{H}+\mathrm{G}^{1}$ & 4 to $5 \%$ & Maupas, 1900 \\
\hline $\begin{array}{l}\text { Rhabditis } \\
\text { aberrans } \text { Krüger }\end{array}$ & $\begin{array}{l}\text { Rhabditis aberrans } \\
\text { (Maupasi group) }\end{array}$ & Rhabditidae & $\mathrm{HPg}$ & & Krüger, 1913 \\
\hline $\begin{array}{l}\text { Rhabditis } \\
\text { anomala } \\
\text { Hertwig }\end{array}$ & $\begin{array}{l}\text { Rhabditis anomala } \\
\text { (Maupasi group) }\end{array}$ & Rhabditidae & $\mathrm{HPg}$ & Unknown & Hertwig, 1922 \\
\hline $\begin{array}{l}\text { Rhabditis belari } \\
\text { Nigon }\end{array}$ & $\begin{array}{l}\text { Mesorhabditis } \\
\text { belari (Monhystera } \\
\text { group) }\end{array}$ & & $\begin{array}{l}\text { see below } R \\
\text { mon-hystera }\end{array}$ & & Nigon, 1949a \\
\hline
\end{tabular}


History of research on C. elegans and other free-living nematodes as model organisms

\begin{tabular}{|c|c|c|c|c|c|}
\hline $\begin{array}{l}\text { Species name } \\
\text { (old) }\end{array}$ & $\begin{array}{l}\text { Species name } \\
\text { (present) }\end{array}$ & Family & Reproduction & Male frequency & Authors \\
\hline $\begin{array}{l}\text { Rhabditis } \\
\text { caussanelli } \\
\text { Maupas }\end{array}$ & \begin{tabular}{|l|} 
Pellioditis \\
hermaphrodita (?)
\end{tabular} & Rhabditidae & $\mathrm{H}$ & $1.4 / 1000$ & Maupas, 1900 \\
\hline $\begin{array}{l}\text { Rhabditis } \\
\text { coronata Cobb }\end{array}$ & $\begin{array}{l}\text { Diploscapter } \\
\text { coronatus }^{4}\end{array}$ & Rhabditidae & $\mathrm{H}$ & $5 / 1000$ & Potts, 1910 \\
\hline $\begin{array}{l}\text { Rhabditis } \\
\text { dolichura } \\
\text { Schneider }\end{array}$ & Oscheius dolichura & Rhabditidae & $\mathrm{H}$ & $9.7 / 1000$ & $\begin{array}{l}\text { Maupas, 1900; } \\
\text { Honda, 1925; } \\
\text { Nigon, 1946; } \\
\text { Nigon, 1949a }\end{array}$ \\
\hline $\begin{array}{l}\text { Rhabditis } \\
\text { duthiersi } \\
\text { Maupas }\end{array}$ & $\begin{array}{l}\text { Rhabditis duthiersi } \\
\text { (Brassicae group) }\end{array}$ & Rhabditidae & $\mathrm{H}$ & $2 \%$ & Maupas, 1900 \\
\hline $\begin{array}{l}\text { Rhabditis } \\
\text { elegans Maupas }\end{array}$ & $\begin{array}{l}\text { Caenorhabditis } \\
\text { elegans }\end{array}$ & Rhabditidae & $\mathrm{H}$ & $1.5 / 1000$ & $\begin{array}{l}\text { Maupas, 1900; } \\
\text { Honda, 1925; } \\
\text { Nigon, 1943; } \\
\text { Nigon, 1949 }\end{array}$ \\
\hline $\begin{array}{l}\text { Rhabditis } \\
\text { guignardi } \\
\text { Maupas }\end{array}$ & $\begin{array}{l}\text { Rhabditis guignardi } \\
\text { (Maupasi group) }\end{array}$ & Rhabditidae & $\mathrm{H}$ & $0.14 / 1000$ & Maupas, 1900 \\
\hline $\begin{array}{l}\text { Rhabditis } \\
\text { gurneyi Potts }\end{array}$ & unclear & Rhabditidae & $\mathrm{H}$ & Not observed & Potts, 1910 \\
\hline $\begin{array}{l}\text { Rhabditis } \\
\text { leptodera } \\
\text { Hertwig }\end{array}$ & $\begin{array}{l}\text { Rhabditis maupasi } \\
\text { (Maupasi group) }\end{array}$ & Rhabditidae & $\mathrm{G}(+\mathrm{FPg})$ & & Hertwig, 1922 \\
\hline $\begin{array}{l}\text { Rhabditis } \\
\text { longicauda } \\
\text { Hertwig }\end{array}$ & $\begin{array}{l}\text { Rhabditis fertilior } \\
\text { (Maupasi group) }\end{array}$ & Rhabditidae & $\mathrm{G}(+\mathrm{FPg})$ & & Hertwig, 1922 \\
\hline $\begin{array}{l}\text { Rhabditis } \\
\text { marionis } \\
\text { Maupas }\end{array}$ & $\begin{array}{l}\text { Rhabditis marionis } \\
\text { (Maupasi group) }\end{array}$ & Rhabditidae & $H+F$ & 13 to $2 / 1000$ & $\begin{array}{l}\text { Maupas, 1900; } \\
\text { Honda, } 1925\end{array}$ \\
\hline $\begin{array}{l}\text { Rhabditis } \\
\text { maupasi Seurat }\end{array}$ & $\begin{array}{l}\text { Rhabditis maupasi } \\
\text { (Maupasi group) }\end{array}$ & Rhabditidae & G & & Hertwig, 1922 \\
\hline $\begin{array}{l}\text { Rhabditis } \\
\text { monhystera } \\
\text { Bütschli }\end{array}$ & $\begin{array}{l}\text { Mesorhabditis } \\
\text { monhystera and } M . \\
\text { belari }{ }^{2}\end{array}$ & Rhabditidae & $\mathrm{FPg}+\mathrm{G}^{6}$ & $6 \%$ & $\begin{array}{l}\text { Belar, 1923; } \\
\text { Nigon, 1949a }\end{array}$ \\
\hline $\begin{array}{l}\text { Rhabditis pellio } \\
\text { Schneider }\end{array}$ & Pellioditis pellio & Rhabditidae & $\begin{array}{l}\mathrm{G} \text { (FPg form " } R . \\
\text { anomala") }\end{array}$ & & $\begin{array}{l}\text { Hertwig 1920; } \\
\text { Hertwig, } 1922\end{array}$ \\
\hline $\begin{array}{l}\text { Rhabditis } \\
\text { perrieri Maupas }\end{array}$ & $\begin{array}{l}\text { Caenorhabditis } \\
\text { perrieri }\end{array}$ & Rhabditidae & $\mathrm{H}$ & $7 / 1000$ & Maupas, 1900 \\
\hline $\begin{array}{l}\text { Rhabditis } \\
\text { schneideri } \\
\text { Bütschli }\end{array}$ & $\begin{array}{l}\text { Rhabditophanes } \\
\text { schneideri }\end{array}$ & Alloionema-tidae & $\mathrm{P}$ & Absent & Maupas, 1900 \\
\hline $\begin{array}{l}\text { Rhabditis } \\
\text { strongyloides } \\
\text { Schneider }\end{array}$ & \begin{tabular}{|l} 
Pelodera \\
strongyloides
\end{tabular} & Rhabditidae & $G$ & & Nigon, 1949a \\
\hline $\begin{array}{l}\text { Rhabditis XIX } \\
\text { (Belar) }\end{array}$ & $?$ & Rhabditidae? & $\mathrm{P}$ & & Belar, 1923 \\
\hline $\begin{array}{l}\text { Rhabditis XX } \\
\text { (Belar) }\end{array}$ & $?$ & Rhabditidae? & $\mathrm{HPg}$ & & Belar, 1923 \\
\hline
\end{tabular}


G: gonochoristic. H: hermaphroditic, self-fertilizing with facultative males. P: parthenogenetic (without sperm). HPg: pseudogamous hermaphrodites (parthenogenesis with self-sperm). FPg: females requiring male sperm for pseudogamy. Present species names in the Rhabditidae from Sudhaus (2011), in the Diplogastridae from Sudhaus and Fürst von Lieven (2003). Note that the family Diplogastridae is nested within Rhabditidae (Kiontke et al., 2007).

1) Among species presently in culture, this mode of reproduction is found in "Rhabditis" sp. SB347 and related species (Félix, 2004; Shakes et al., 2011).

2) It is unclear whether Belar and Nigon observed the same species. Belar called it $R$. monohystera (lapsus for monhystera) and Nigon described a new species $R$. belari, arguing that it may be the same as Belar, but different from the published R. monhystera Bütschli (Nigon, 1949).

3) The dauer larvae of $R$. caussanelli Maupas appears to wave in tubes, formed by their L2 cuticle (Maupas, 1899). However Pellioditis hermaphroditica does not display this behavior (W. Sudhaus, personal communication).

4) Diploscapter spp. presently in culture are parthenogenetic without sperm (Lahl et al., 2006). In this group, Protorhabditis sp. JB122 does have sperm (M.-A. Félix, unpublished observations) while being recorded to have a single pronucleus (Brauchle et al., 2009).

5) What is presently in culture under the name of $O$. dolichura is a species that is much less common than $O$. tipulae. It is doubtful that all early works were based on the isolation of the same rather infrequent species. It is likely that several selfing Oscheius species were lumped into "O. dolichura" (Notes by M.-A. Félix).

6) Among species presently in culture, this mode of reproduction is found in Mesorhabditis sp. PS1179 (M. Delattre and M.-A. Félix, unpublished).

\section{Appendix 2. The C. elegans Bergerac strain.}

Bergerac is a town in the SouthWest of France, known to some for its wine or Cyrano de Bergerac, but to $C$. elegans scientists of a certain generation for a $C$. elegans strain with active transposons (but see below).

The present Bergerac strain was isolated in 1944. While living in Bergerac in 1941-44, Nigon isolated $C$. elegans on two occasions from the garden of the house he lived in (see Biography, Section 18.4). The first strain was collected in October 1941; it was used in the work published in 1943 (Nigon, 1943) and discontinued at the end of 1942. A second strain was isolated in March 1944 in the same garden and is that known today as Bergerac. This second strain was sent to several laboratories that gave it various strain names, some of which indicate the culture location (Be for Berkeley, $\mathrm{BO}$ for Boulder), others are according to the later standardized strain nomenclature (CB4851, DR1344, RW7000, etc.). This sharing occurred before the freezing of C. elegans strains was implemented. Therefore, as for the Bristol strain (McGrath et al., 2009; McGrath et al., 2011; Sterken et al., 2015), the Bergerac subclones diverged from the ancestor and from each other through the acquisition of new mutations (Figure 23). The present appendix aims at reconstituting this history, particularly pointing out that: 1) the temperature-sensitivity of Bergerac is not primarily due to transposition but to a temperature-sensitive point mutation in the zyg-12 gene, common to all Bergerac subclones; and 2) the high level of Tc1 transposition activity that many C. elegans scientists associate with Bergerac only applies to the BO/RW7000 sublineage. 
History of research on C. elegans and other free-living nematodes as model organisms

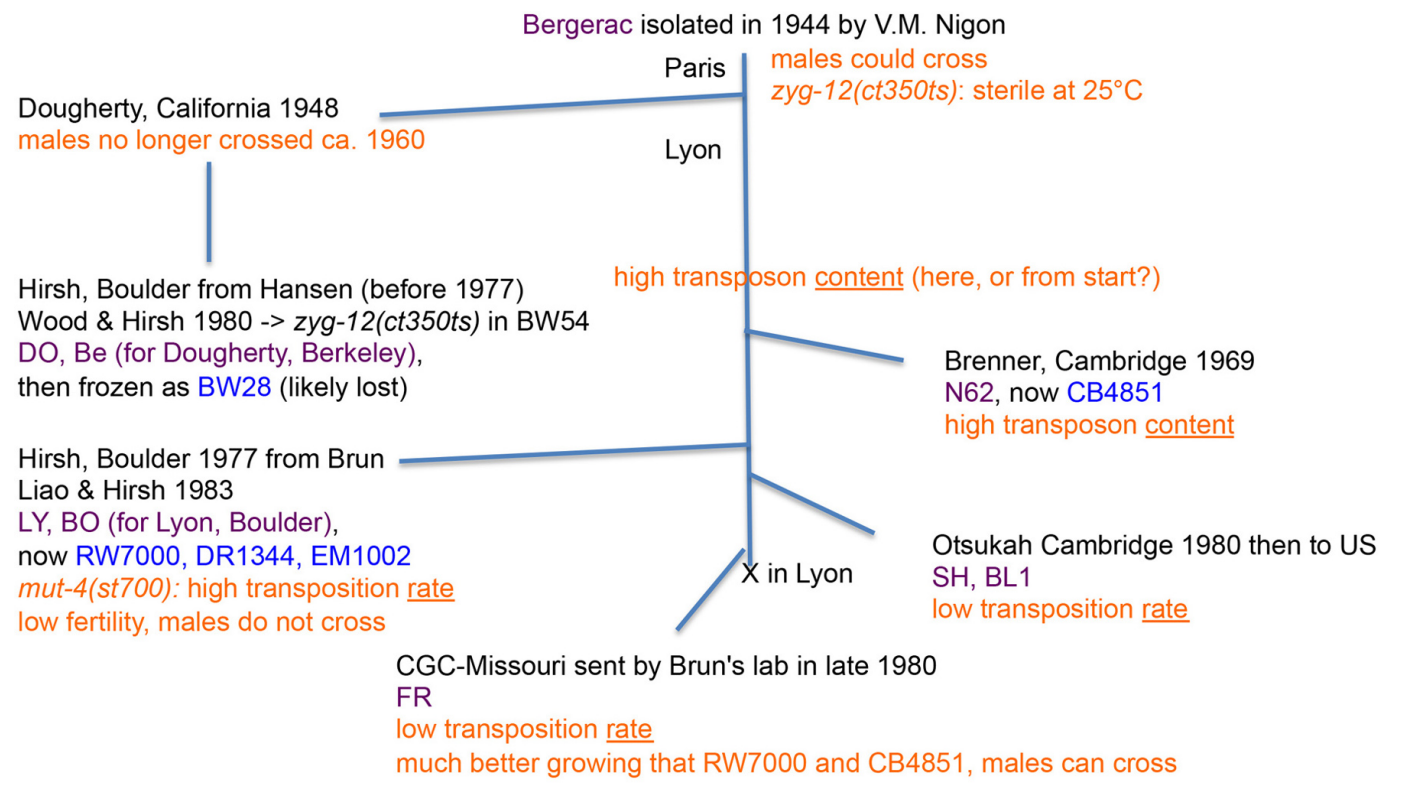

Figure 23. Tentative history of the Bergerac strain. Early strain names are in purple, recent strain names with lab codes are in blue. The line kept in Lyon by Nigon, then Brun, is indicated as the vertical central blue line, with branching sublineages. Strain names used before standard strain nomenclature are in violet, those with standard strain nomenclature are in blue.

\subsection{France FR sublineage}

The original strain and its descendant lineage in Lyon were temperature-sensitive, displaying abnormalities from 23-24 ${ }^{\circ} \mathrm{C}$. Males mated well and were used for crosses (Nigon, 1949a; Dion and Brun, 1971). The strain was maintained in Lyon at low temperatures and remained healthy. It was sent to the CGC in 1980, and frozen under the name of Bergerac FR. Liao et al., (1983) state that "The Bergerac FR strain has male fertility levels and brood sizes comparable with Bristol N2, while the Bergerac LY strain has low male fertility and brood sizes." The strain is no longer available at the CGC and was kindly provided to M.-A. Félix by Mark Edgley and Donald Moerman. It is indeed healthy, compared to the other sublineages mentioned below (observations by M.-A. Félix.).

\subsection{Berkeley sublineage}

Nigon shared the Bergerac strain with Dougherty in 1948. After several years of culture (perhaps in part in liquid), males of the California strain could no longer mate well (Fatt and Dougherty, 1963). After Dougherty's death, Eder Hansen gave a subclone to David Hirsh (University of Colorado, Boulder) who used it for further studies of heat sensitivity (Wood et al., 1980). William (Bill) Wood built a strain (BW54) where the heat-sensitive locus from Bergerac was introduced by repeated crossing into the N2 background; this strain was later used for the identification of the zyg-12(ct350ts)II mutation by Malone et al. (2003). The zyg-12 mutation is also present in FR, RW7000 and CB4851 (genotyping by A. Richaud and M.-A. Félix), indicating that the nucleotide substitution was likely already present in 1944 (or appeared shortly thereafter). This temperature-sensitive allele of zyg-12 explains the temperature sensitivity of the Bergerac strain (see Section 9.3). The Bergerac Be strain in Johnson and Hutchinson (1993) and the Bergerac DO mentioned in Levitt and Emmons (1989) seem to correspond to this sublineage via Dougherty in Berkeley.

\subsection{Cambridge sublineage}

The Bergerac strain was sent by Nigon to Brenner a first time in 1966 and again in 1969 when the first one was lost (Nigon, 1966/1970). Brenner called it N62. Once the strain nomenclature was standardized (Horvitz et al., 1979), this strain was renamed CB4851 (Hodgkin and Doniach, 1997). CB4851 is phenotypically "sick" (more so than FR) and has a high spontaneous male production (Hodgkin and Doniach, 1997). CB4851 also has a high Tc1 transposon content (Hodgkin and Doniach, 1997). 


\subsection{Boulder BO sublineage}

The BO/RW7000 Bergerac subclone is the best known to $C$. elegans scientists of the generation that was active in the 1980-90s, when it was used for genetic mapping using transposons and for mutagenesis using transposase activity (see Section 12.2). This BO sublineage originated from a gift of a Bergerac culture by Jean Brun to David Hirsh in Boulder, CO in 1977 (Moerman and Waterston, 1984). This sublineage is a very sick derivative, which bears various names as it moved among laboratories and as strain nomenclature evolved: LY ("Lyon"), BO ("Boulder"), RW7000, DR1344, EM1002, and DP13 (Liao et al., 1983; Moerman and Waterston, 1984; Emmons and Yesner, 1984; Williams et al., 1992). While Hirsh's laboratory used the Dougherty Bergerac strain for zyg-12(ts) isolation, they used that originating from Brun in 1977 (BO) as a source of transposons. The BO subclone was found to contain $\sim 200-500$ copies of the Tc1 transposon, compared to $\sim 30$ for N2, and these differences between BO and N2 were used as polymorphisms for genetic mapping (Emmons et al., 1983; Egilmez et al., 1995; Korswagen et al., 1996). In addition, the BO sublineage evolved an increased Tc1 transposition activity in the germ line, at least 100-fold over that of C. elegans N2 and Bergerac FR (Moerman and Waterston, 1984), resulting in the appearance of phenotypic mutants in the culture (Mutator phenotype). This transposon activity was used for mutagenesis and tagging (see Section 12.2). This increased transposon activity likely occurred through the activation of a transposon copy, forming the mut-4(st700) mutator allele on chromosome $I$, which then hopped to other chromosomes, giving rise to mut-5(st701)II and mut-6(st702)IV (Mori et al., 1988). These mutator alleles have not been molecularly identified. The Tc2 transposon also has an increased copy number compared to Bristol in Bergerac subclones, including FR (Levitt and Emmons, 1989), while Tc3, Tc4, and Tc5 do not (Collins, 1989; Yuan et al., 1991; Collins and Anderson, 1994). Since CB4851 also has a high Tc1 transposon content (Hodgkin and Doniach, 1997) and $\mathrm{BL} 1 / \mathrm{SH}$, a later derivative from the Lyon lineage, has some detectable transposition activity (Moerman and Waterston, 1984), it appears likely that the ancestor of CB4851, BO/RW7000, and BL1 and maybe the original isolate already had a somewhat elevated level of Tc1 transposon and transposition activity, which further increased in a dramatic fashion in the BO/RW7000 sublineage. In sum, the high transposition activity and extreme strain sickness only apply to the BO/RW7000/etc. derivatives, not to the FR strain that was kept in Lyon.

Note that the high-Tc1 content strains DH424, TR403, and CB4555/DR1349 that were considered to be wild isolates of $C$. elegans turned out to be recombinants between N2 and a Bergerac subclone (apparently the same recombinant, likely a lab contamination from the start; McGrath et al., 2009).

This account leaves several pending questions:

1) How was the Tc1 transposase activity activated in the BO sublineage?

2) Is there a relationship between the zyg-12 mutation and the transposon activity?

3) Whether wild strains of $C$. elegans with elevated transposon activity exist remains to be studied.

\section{Appendix 3. Biographies}

Below are brief biographies (in chronological order by birth date) of Emile Maupas (Section 18.1), Paula Hertwig (Section 18.2), Karl Belar (Section 18.3), Ellsworth Dougherty (Section 18.5), and Jean-Louis Brun (Section 18.6)-and an autobiographical essay by Victor Marc Nigon (Section 18.4)-focusing on the circumstances of their work with nematodes. We were unable to find information about Eva Krüger (University of Freiburg, Germany) and Hikokura Honda (University of Chicago, then University of Pennsylvania, USA) and hope that someone may do so in the future. 


\subsection{Emile Maupas (1842-1916)}

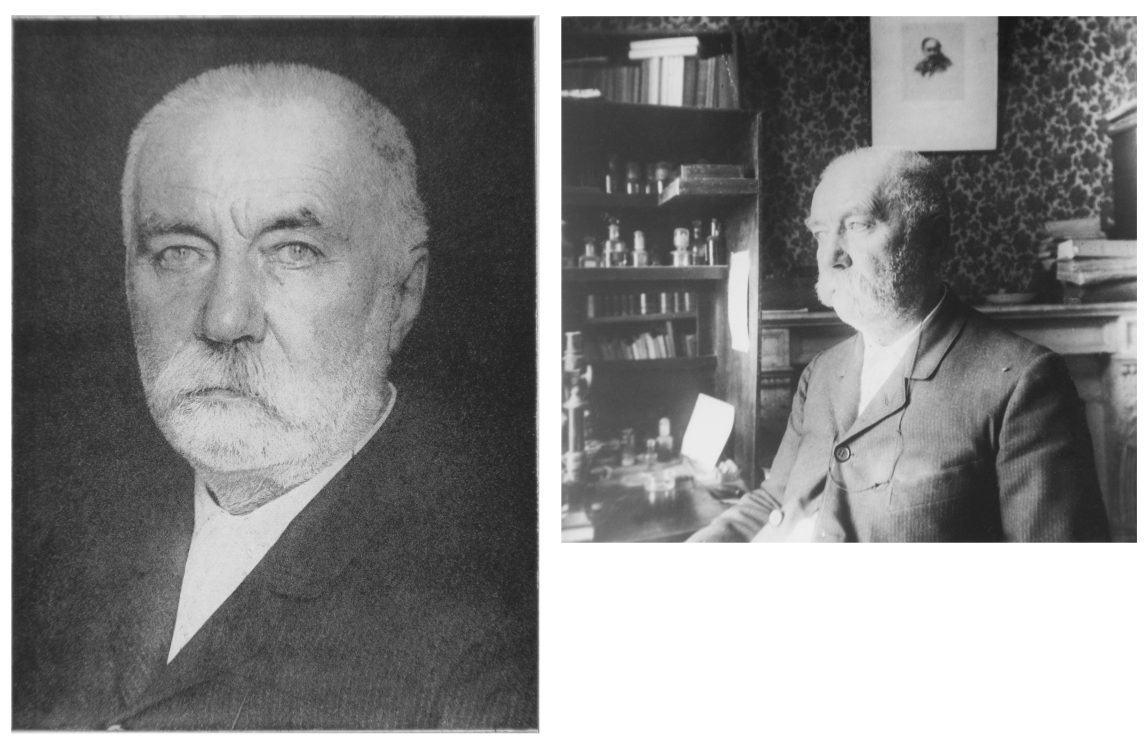

Figure 24. Emile Maupas. Pictures from 1912, on the right at home in front of his microscope and cultures. Archives of the Academy of Sciences (France), with permission.

Emile Maupas studied at the Ecole des Chartes in Paris to become an archivist, thus quite far from biology; his thesis concerned legislation during the wars of the Middle Ages. He first worked as an archivist in Cantal in the center of France, and then moved to Algiers (a French territory at the time) in North Africa, where he stayed from 1871 until his death in 1916. He became the Head of the National Library of Algiers. His afternoons were devoted to the Library, and the rest of his time to his activities as an "amateur" biologist. For the latter, he worked at home, a modest 3-room dwelling in the neighborhood of Bab el Oued, overlooking the Mediterranean Sea. The microscope was in front of the window; the rest of the "laboratory" (a few kitchen plates with his cultures) could fit on the fireplace shelf (right picture; see Caullery, 1937).

Maupas' curiosity focused on the biology of small organisms such as ciliates, nematodes, rotifers, and oligochaetes, which he isolated from soil or freshwater. He developed culture methods to observe their development, reproduction, and sexuality-a quite unusual endeavor at a time when the study of morphology predominated. He thus can be considered as one of the first biologists who, with rudimentary equipment, applied the experimental method to basic biological questions, similar to that developed in physiology by Claude Bernard.

Maupas is perhaps best known for his studies of lineage degeneration in ciliates, where he showed that a given mitotically reproducing clone had a finite lifetime, unless sexual reproduction occurred. He carefully observed their sexual reproduction and correctly interpreted the exchange of micronuclei. In the context of the 1880s, observing sexuality in a single-cell organism, and the role of the nucleus therein, was of major significance in attributing to the nucleus a role in heredity. Maupas also studied sexuality in rotifers, and turned, in the last 20 years of his life, to using nematodes as model organisms. His interest for nematodes concerned their lifecycles and the evolution of their diverse modes of reproduction (see main text). As an aside, he described and named the several new species that he isolated and observed, including C. elegans (then Rhabditis elegans), clearly his favorite species in the 1900 opus.

Despite Maupas' institutional and geographical isolation, the meticulousness of his experiments and the relevance of his interpretations attracted the attention of great biologists, such as Otto Bütschli (another "protistologist" with an inclination for nematodes), then Professor in Heidelberg, who obtained for Maupas the title of Doctor Honoris Causa of the University of Heidelberg in 1903. The French Academy of Sciences elected Maupas as a Correspondent in 1901 and awarded him the Grand Prize for the Physical Sciences for his work on development and reproduction of nematodes. In 1932, the French zoologist Maurice Caullery erected a plaque in his honor on his house in Algiers. He then gave a laudatory speech (Caullery, 1937), mentioning that he had never met Maupas, but that Bütschli had expressed to him in 1891 his admiration for Maupas. Caullery was a key figure of French zoology at the time, and the founder and head of the "Laboratoire d'Evolution des Êtres Organisés" (Laboratory of Evolution of Organized Beings) at the University of Paris. He was succeeded in this role by Pierre-Paul Grassé, who hosted 
Victor Nigon in his first years in Paris in this same (still standing) building on Boulevard Raspail (Caullery was then retired but still around).

A high school bears Maupas' name in Vire, Normandy (his birthplace) and in Skikda (formerly Philippeville, in Algeria). A Maupas medal is still being awarded for great achievements in the ciliate scientific community.

\subsubsection{References by Maupas on nematodes}

Maupas, E. (1899). La mue et l'enkystement chez les nématodes. Arch. Zool. Exp. Gen. 7, 563-628. Article

Maupas, E. (1900). Modes et formes de reproduction des nématodes. Arch. Zool. Exp. 8, 463-624.

Maupas, E. (1915a). Sur un Champignon parasite des Rhabditis. Bull. Soc. Hist. Nat. Afr. Nord. 7, 34-49. Abstract

Maupas, E. (1915b). Un nouveau Rhabditis. Bull. Soc. Hist. Nat. Afr. Nord. 7, 51-52. Abstract

Maupas, E. (1916a). Nouveaux Rhabditis d'Algérie. CR Soc. Bio.1 79, 607-613.

Maupas, E. (1916b). Sur le mécanisme de l'accouplement chez les Nématodes. CR Soc. Biol. 79, 614-618.

Maupas, E. (1919). Essais d'hybridation chez des nématodes. Bull. Biol. Fr. Belg. 52, 466-498.

\subsubsection{References on Maupas}

Jordan, C. (1916). Notice nécrologique sur M. Maupas. C R Acad. Sc. Paris 163, 55-56.

Caullery, M. (1937). Inauguration d'une plaque commémorative apposée sur la maison habitée par Emile Maupas à Alger, le mercredi 6 avril 1932. In Notices et discours, A.d. Sciences, ed., pp. 380-389.

Loison, L. http://biosex.univ-paris1.fr/dossiers-thematiques/maupas/

Nanney, D.L. http://www.life.illinois.edu/nanney/maupas/maupas.html

\subsection{Paula Hertwig (1889-1983)}

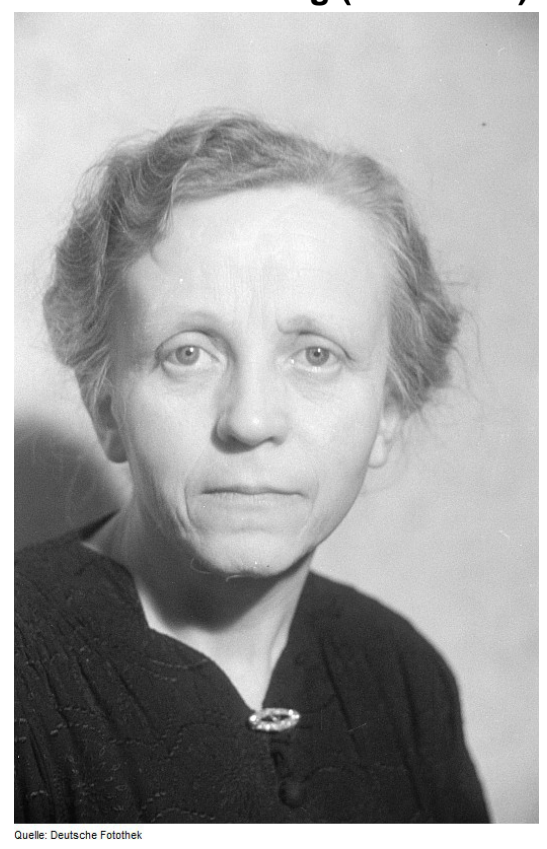

Figure 25. Paula Hertwig. from https://www.hu-berlin.de/de/ueberblick/geschichte/wissenschaftlerinnen/hertwig 
Paula Hertwig grew up in a family of famous biologists: Oscar Hertwig was her father and Richard Hertwig her uncle. Throughout her life, she was also close to her brother Günther Hertwig, with whom she shared some scientific projects. Paula Hertwig studied in Berlin and obtained her doctorate in 1916. Her first publication in 1911 reported on the abnormal divisions of Ascaris eggs upon exposure to radium. She studied many organisms. Among them, she isolated free-living nematodes to study the cellular basis of meiosis and fertilization and variations in modes of reproduction. In particular, she studied a spontaneous mutant observed in Rhabditis pellio (see main text). Most remarkably, based on this work, in 1919 she was the first woman in Germany to obtain the Habilitation. She became Privat-Dozent at the University of Berlin, despite a law barring women from obtaining faculty positions. Another law also excluded married women from state employment, and she did not marry.

In 1921, she became Assistant at the Institute of Genetics of the Agricultural College, which was directed by the plant geneticist Erwin Baur. In particular, she studied the genetics of a hermaphroditic variant of Melandrium (Silene dioica). She also had a long-standing interest in the hybridization of animal species.

From 1927 to 1945, she was Extraordinary (Adjunct) Professor of Genetics at the Medical School of the University of Berlin. She was politically active, elected in 1932 as representative of the liberal German Democratic Party in the Prussian Parliament. After the war, she and her brother chose to live in the German Democratic Republic (GDR = East Germany). In 1948, she obtained a Chair in Genetics at the Medical School of the University of Halle (GDR), where she also became the Dean. She headed a new Institute of Genetics in this university. When Lysenko obtained political support in his rejection of genetics, which resulted in the persecution of geneticists in the Soviet Union, she expressed herself against him. She was elected a member of the Leopoldina, the German Academy of Sciences (then in East Germany), in 1953. After the death of her brother, she retired in 1972 to Villingen in the Black Forest in West Germany and, in 1973, became Doctor Honoris Causa of the University of Heidelberg.

\subsubsection{Articles on nematodes by P. Hertwig}

Hertwig, P. (1911). Durch Radiumbestrahlung hervorgerufene Veränderungen in den Kernteilungsfiguren der Eier von Ascaris megalocephala. Arch. Mikrosk. Anat. 77, 301-312. Article

Hertwig, P. (1920). Abweichende Form der Parthenogenese bei einer Mutation von Rhabditis pellio. Arch. Mikrosk. Anat. 94, 303-337. Article

Hertwig, P. (1922). Beobachtungen über die Fortpflanzungsweise und die systematische Einteilung der Regenwurmnematoden. Z. wiss. Zool. 119, 539-558.

\subsubsection{Articles on P. Hertwig}

Gerstengarbe, S. (2003). "[...] kenntnisreich, überlegt, kritisch gut veranlagt und von guter Darstellungsgabe”- die Genetikerin Paula Hertwig. Biospektrum 4/2003, 378-380. http://www.biospektrum.de/blatt/d_bs_pdf\&_id=933900

Gerstengabe, S. (2012). Paula Hertwig - Genetikerin im 20. Jahrhundert. Eine Spurensuche. Acta Historica Leopoldina Nr. 58.

http://www.epigenesys.eu/en/science-and-you/women-in-science/795-paula-hertwig 
18.3. Karl Franz Joseph Belar (1895-1931)

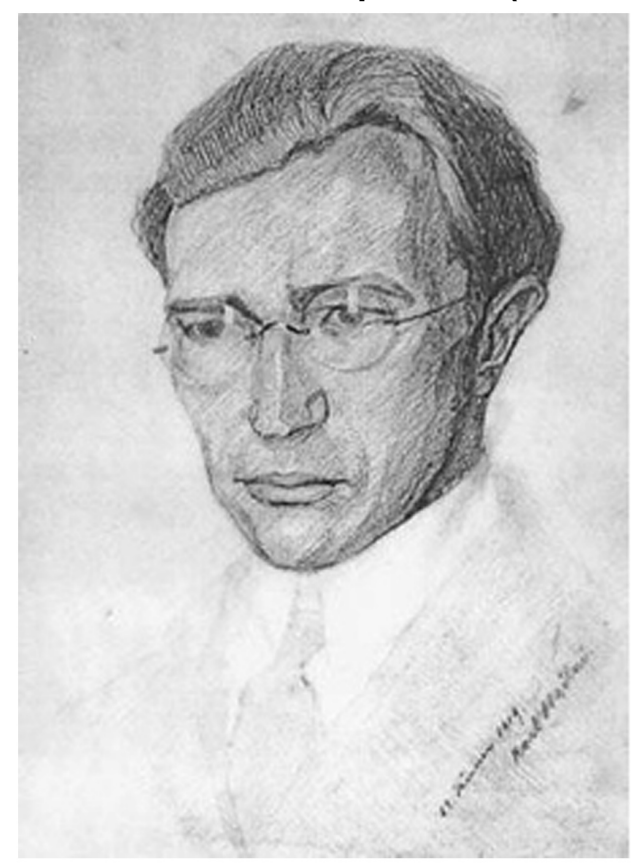

Figure 26. Self-portrait, Karl Belar. From http://pl.wikipedia.org/wiki/Plik:Belar.JPG

Belar (pronounced Bielarch) grew up in Vienna, in an intellectual environment. He studied zoology, had access to his brother-in-law's private laboratory for his research, and defended his doctorate in protistology in 1919. From 1915 to 1918, he served in the Austrian army. After his doctorate, he was invited to Berlin by Max Hartmann, director of a section at the Kaiser Wilhelm Institute and protistologist, and became his assistant. In 1925, he obtained the title of Privatdozent of the University of Berlin. His main work remained centered on protists, although he became interested in the use of nematodes. In 1929, he took an invited professorship at The California Institute of Technology (Caltech) in the new Division of Biology headed by Thomas H. Morgan, and spent nearly two years in Pasadena, CA, USA. His main material there was the embryo of Urechis, a marine annelid. Just when he was due to return to Germany, he died in a car accident on a trip to the Mojave desert, where he painted, one of his favorite hobbies.

Like Bütschli and Maupas, Belar mostly worked with protists, studying nematodes and other animals on the side. Although being best known for his work in protistology, he had more general interests and was able to link his work in protistology to general cytology matters. In protists, such as Actinophrys sol, he studied cell division and could draw parallels with the events of mitosis observed in animals. His later work concerned the mechanistic aspects of cell division, including spindle mechanics. He was well-known for the quality of his drawings, and was described as a rigorous and critical mind with wide-ranging intellectual interests, and especially as a gifted amateur artist.

His interest in consistent and varying features of cell division, particularly meiotic divisions and first mitotic divisions, seems to have drawn him to Rhabditis as a model. Following Eva Krüger and Paula Hertwig, he studied several Rhabditis species where the sperm was required for fertilization yet sperm chromosomes degenerated upon entry into the egg (pseudogamy; see Main text).

He wrote several general works concerning cytological questions in genetics, including "Cytologische Grundlagen in Vererbungswissenschaft" (1928; Cytological bases in the sciences of heredity). This book was a reference for V.M. Nigon; in the 1940s, it was the only synthetic work concerning cytological approaches to heredity.

When V.M. Nigon asked Professor Vandel for a subject for his Diplôme d'Etudes Supérieures (Master), which he could accomplish while living $200 \mathrm{~km}$ from the university, Vandel referred to Belar's work. He mentioned a visit to Belar's laboratory, where Belar had shown him his nematode cultures and had told him about his nematode work. 


\subsubsection{References by Belar on nematodes}

Belar, K. (1923). Über den Chromosomenzyklus von parthenogenetischen Erdnematoden. Biol. Zbl. 43, 513-518.

Belar, K. (1924). Die Cytologie der Merospermie bei freilebenden Rhabditis-Arten. Z. f. Zellen und Gewebelehre 1, $1-21$.

\subsubsection{References on Belar}

Stern, C. (1931). Karl Belar zum Gedächtnis. Die Naturwissenschaften 46, 921-923.

Jaenicke, L. (2004). Karl Franz Josef Belar, October 14, 1895-May 24, 1931. A sharp eyed and pencilled early genius of caryogenetics, unifying general caryology with chromosomal inheritance. Protist 155, 459-469. Abstract Article

\subsection{Victor Marc Nigon (11 October 1920 - 5 July 2015)}

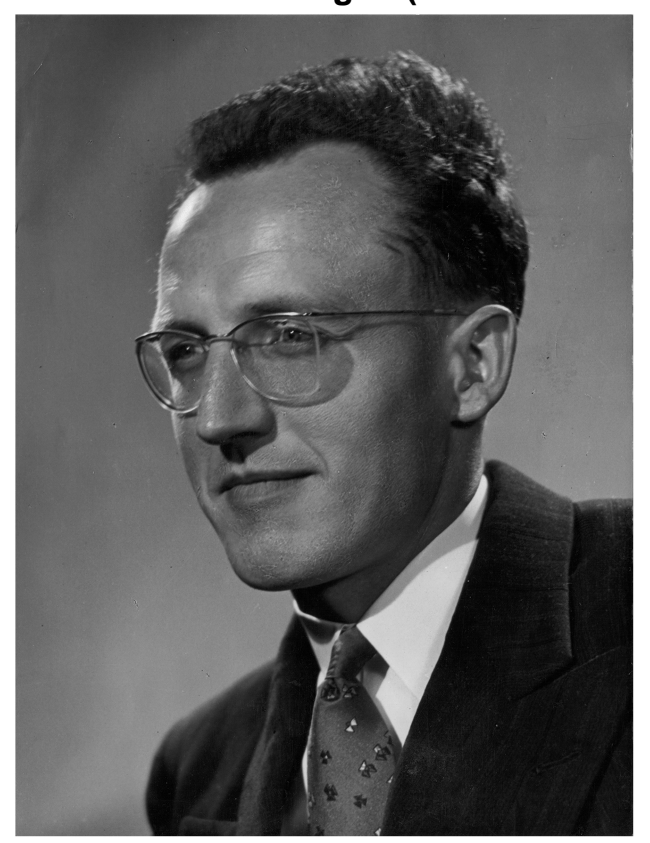

Figure 27. V.M. Nigon in 1952, personal collection.

Short professional CV: Starting in October 1944, researcher at the Centre National de la Recherche Scientifique (CNRS), working at the Laboratory of Evolution in Paris, directed by Professor Grassé. From October 1947 to September 1949, "Chef de Travaux" (Lecturer) at the Sorbonne. In October 1949, "Maître de Conférences" (Reader) in Lyon, then Professor in 1953. In Lyon, he set up courses in Genetics and Embryology, the Section of General and Applied Biology in 1962 (associated with the CNRS), then a Laboratory associated with the Institut National de la Recherche Agronomique (INRA). This latter was devoted to avian leukemia viruses. These viruses were used as vectors by Nigon et al. to study hemoglobin synthesis in cultures of avian blood precursor cells. From 1970 to 1980, Director of a French-Algerian Programme on thalassemia, financed by the Foreign Affairs Ministery. Emeritus from 1986 to 1989. During retirement, overseas projects from the Foreign Affairs and INRA: Japan in 1991 and 1992, Bolivia from 1994 to 1999. Co-director of the Bolivian Institute for High-Altitude Biology 1995-1999. Honorary Professor of the University of La Paz in 1998.

How did you start working with Rhabditis? This question was often asked of me. It was an exceptional combination of circumstances. 
I was born in 1920 in France, in the department of Moselle. Local history played a large influence in my personal life. Europe had been largely united in the Roman Empire, but after Charlemagne's reign, it fell into pieces, each one under the power of local kings or princes. The territory of Moselle was a part of the Dukedom of Lorraine, where the official language was French, but at its Northern border in Moselle a small part of the territory traditionally used a German dialect and was thus known as "bailliage d'Allemagne". My family had lived in this territory since the 16th century. While speaking at home a German dialect, some family members participated in the French-speaking administration of Lorraine. It took over ten centuries to rebuild large countries like France and Germany. Lorraine was incorporated into France in the 17th and 18th centuries. The unification of Germany occurred later, in the second half of the 19th century, and strove to include all German-speaking regions. After France's defeat in 1871, together with Alsace, Germany thus annexed the Moselle department. However, during years of association with French-speaking Lorraine, the German-speaking Lorraine had adopted French traditions, such as the practice of the Roman Catholic religion (while most regions of Germany were following the Lutheran Reform). For such reasons, the annexation of a part of Lorraine by Germany was not universally accepted and Moselle returned to France after the German defeat of 1918. In 1940, the German Nazi power decided unilaterally to re-annex Moselle. From past experience they knew that annexing a French-speaking part would result in local opposition. They thus decided to expel to France 200,000 French-speaking people in September and October, 1940. My parents, over 60 years of age and partly German-speaking from childhood, could stay. I easily spoke German and had visited a large part of Germany in 1937 and 1938. I appreciated German literature, philosophy, and science. But I gained there a profound aversion to Hitler's politics. Thus, my decision in 1940 was to avoid a German status. My parents approved of my choice. When asked by the German administration they declared that I had been transferred overseas by the French army. They advised me to avoid any contact with the German police, who continued to inquire about me. If I fell into their hands, I would be obliged to choose: either my return to German Lorraine, or the departure of my parents. I thus had to live in the highest discretion, avoiding any contact with Germans. This obliged me to live in the non-occupied zone of France (Note: roughly the South half of France, but the demarcation line run diagonally in the SouthWest, leaving Bordeaux in the occupied zone while Bergerac and Toulouse were in the non-occupied one). This history was a key background, to which I will return below, for why I ended up working on C. elegans, while stranded in Bergerac as a consequence of the war and my Lorraine origins.

From the age of 12, I had decided to become a scientist. At $16 \mathrm{I}$ had entered the University of Paris as a medical student (1936). My plan was to prepare to enter the Pasteur Institute in Paris. I had developed a passion for tropical diseases. Their study would conciliate my passion for scientific research, my social inclinations towards poor people, and my taste for travel. Yet I got rapidly disappointed by the archaic character of medical studies at French Universities. During my second year I met a Laboratory head of the Pasteur Institute, who explained to me that only half of the personnel had a medical degree, while the other half had a science degree. My decision was immediate-the next year, I abandoned the medical curriculum and registered at the Faculty of Sciences, which conformed better to my expectations.

In 1940, my parents, devoid of French money, could no longer finance my studies. I thus had to earn my living, while continuing my studies. At the University of Lyon (in the non-occupied zone), I obtained a low-paid position in Geology, a discipline that did not interest me. I got married, very early. The mother of my spouse died in March 1941. Her father asked my wife, who was the oldest of his children, to settle close to him to help raising his younger children. He offered us a house, a support through his relationships with surrounding farmers and, for me, help in finding private lessons to teach. He lived in Bergerac, a small town that was very appropriate for my need to be discreet. The only difficulty was the distance to the University of Toulouse, the closest one in the non-occupied zone-practically I could not attend the courses. I went to Toulouse to consult Professor Vandel who headed the sector of Animal Biology. He received me with a great understanding and the willingness to help me. I am happy to express here a tribute of gratitude to his memory. Professor Vandel gave me a list of works to study and introduced me to his best students, who gave me their notes on the classes and practicals. I complemented these documents with popular books about genetics and embryology. I passed the exam in October 1941, being the top-ranked student, with the best possible mark.

I could no longer hope to work in the Pasteur Institute, located in German-controlled France. With my wife, we prepared a project for me to teach in French schools in a foreign country, possibly the United States. After the war, I might have been able to obtain such a teaching position and take part in a research lab in the United States. Before this, my next academic step was a year dedicated to work in a laboratory, on a subject that usually implied reproducing data from the literature, with a written dissertation and an oral examination at the end. Unable to pursue work at the University, I had to perform this experimental study at home with rudimentary instrumentation. I proposed this to Professor Vandel, who immediately accepted. He told me about Maupas, of whom he had helped 
publishing a posthumous article, and of Belar, whom he had met. He was attracted by the simple requirements of the Rhabditis culture, which I could likely manage. Apparently, he had not grasped the further potential of this material. He likely hoped that, by repeating these experiments, I would confirm, and maybe elaborate on the ambiguous results of Maupas on crosses. I immediately accepted.

The first hermaphroditic nematode that I isolated turned out to be the famous Caenorhabditis elegans described by Maupas and I did not search further. I placed some garden soil in a kitchen plate and maintained its humidity by covering it. On the plate, I added diverse items as traps. I remember using carrot slices and also earthworm pieces from the same garden, cut with scissors! Two or three days later I transferred the trap with some surrounding soil onto a Petri dish with Knop agar. I waited until the Petri dish was swarming with larvae and tried to transport to a new plate the smallest possible number of animals. One day later, they were adults and visible with the naked eye, which allowed me to catch one with the tip of a scalpel and seed a new plate with a single individual. All this without a dissecting microscope at hand: I only had an old Stiassnie microscope offered by my parents at the beginning of my medical studies. By proceeding in this manner, I hoped to select a hermaphroditic species, which I could monitor by observing with my microscope that there were only females. I then needed to hunt for the rare males that would allow me to determine the species. I do not remember having had to proceed through numerous identifications to isolate the C. elegans that I was looking for, based on the initial data by Maupas.

My readings of the prior studies struck me by the rudimentary character of the culture methods that were used. They contrasted with what I knew of the rigorous methods used by Morgan for Drosophila. I thus decided to develop for Rhabditis a more rigorous culture method. Within two months, I invented a method of breeding isolated animals on a drop of agar, bringing nutrients to the culture, with daily transfers of the parents to fresh medium, which, in a few weeks, gave males in great quantity. These results impressed Vandel and I wrote a short article relating them (Nigon, 1943).

After my diploma, I had to participate in "Chantiers de Jeunesse", which corresponded to practical work that replaced military training in the "free" zone of France. But I soon had to face a new danger: at the beginning of 1943, the French government decided to send those of my age to Germany as compulsory workers. The German police would easily then identify my origin from the German-speaking part of Lorraine and I immediately decided to prevent this. With the help of local medical doctors, I simulated an accident with a broken foot. This resulted in six weeks in the military hospital with my foot in a cast. When I came back to the Chantier, all of those of my age had been transferred to Germany. I had prepared a possible fallback in the maquis in case of danger.

Some time later, I asked Vandel about future possibilities. Naively, I told him of my project to emigrate to the United States. He then informed me about the projects that, with other Professors, doubtless in Resistance networks, he planned for France once liberated. At that time, Germany had lost Stalingrad and was starting to retreat. Many French people were beginning to think that a German defeat was conceivable. Vandel told me that two months after the installation in Paris of a Free French Government, the CNRS would reopen, creating jobs for young scientists. So I should be in Paris at the time of the liberation. He had already talked about me to Parisian colleagues who were ready to host me. After my studies, I had obtained a well-paid job as a chemist in a company in Bergerac, which allowed us to spare the necessary sum for me to spend six months in Paris, while my wife and children would stay in Bergerac, with her father's family. I thus moved to Paris, alone, in April 1944.

I was received by Professor Grassé, who installed me in a laboratory, with dream equipment compared to what I had at home in Bergerac. Paris was liberated in August. On $1^{\text {st }}$ October, my project on Rhabditis was accepted by the CNRS. I was recruited as a research trainee with a monthly salary of 1000 Francs, a fourth of my salary in Bergerac. It was difficult to support a family with this amount. My wife managed to do so, to the amazement of my friends. In sum, so far my path towards Rhabditis resulted from an encounter with Vandel, with, for me, difficult conditions of living. For Vandel, the lineage was Maupas and Belar, yet apparently without a deep conviction concerning its interest. It was only through my work in Paris that the richness of the Rhabditis project struck me.

In 1948, I hosted a visit from Ellsworth Dougherty, who spent six months with me, learning to use the experimental method as scientific approach. Our discussions helped us to enlarge the scope of our projects. The misfortunes of the war, the extreme poverty, the cobbled-together equipment, the life with rudimentary comfort, the political climate, etc.: all this deeply marked scientific research in France at that time. Today's scientists cannot imagine the influence of these work conditions and the approaches of researchers in this period. When Ellsworth came to France, he told me of his surprise at this work atmosphere, which he had never experienced. These ascetic conditions, according to him, played an important role in stimulating the creativity of scientists. He had never worked with such intensity and concentration. 
I defended my thesis (Ph.D.) in the spring of 1948. On 1st October 1949, I was appointed Lecturer at the University of Lyon. Access to an academic position entailed establishing a research program, with the participation of young researchers. I had reached, at this point, a quite clear view of the potential of Rhabditis. I had some experience with cytology and saw a future with genetics. Yet, in line with a French tradition, the genetics of the time, including the remarkable results of Morgan, appeared to me to suffer from major problems, resulting especially from the abstract character of the gene. It allowed the development of unverifiable theories, such as the theory of polygenes. A way to avoid this abstract theory was through the birth of what has become Molecular Biology; the genetic role of DNA was just proven at this time by Avery's experiments on bacteria. I was thus wondering how to access molecular biology starting from Rhabditis. The only answer I could give was to associate, in one program, several experienced researchers-at least a geneticist and a biochemist. Around me, I searched for candidates and only found young students, without experience. Most experienced researchers considered molecular biology as an unreachable utopia. Nevertheless, I searched for the support of influential Professors. This again was a failure. Evidently, this project could not succeed in France. The choice was limited to continuing on nematodes, forgetting, at least for a while, molecular biology. Or, follow my quest towards molecular biology by testing other material, on which more advanced knowledge could shorten the way towards molecular biology. In 1953, I chose the second path.

At that time, I had already started to train some students in research. Two of them wished to continue on the nematodes: Jean Brun, who worked on this subject until his death, and Pierre Guerrier, tied to his video camera. Later, Guerrier continued to study the first cleavage divisions, this time in marine Spiralia.

In the 1953-1965 period, I had defined an aim-to find transmissible characters in a eukaryotic species, with which one should be able to study the path from the gene to the results of its expression. Following this aim, I tried several species, including Drosophila, the silkworm, and the green alga Euglena. I performed this research with young students. If, after a few years, I could not obtain the desired results on one species, I moved to another. Several of my young collaborators then chose to continue on the material that I had left behind. The sought-after result came with the work on Euglena bleached by culturing them in the dark and brought back to light. One could observe the formation of chloroplasts and perhaps determine the mechanism. We examined ground extracts of greening cells after ultracentrifugation on a sucrose gradient. I immediately recognized the signal of polysomes, already described in bacteria, sea urchin eggs, and erythropoietic cells. This was one of the results I was looking for, as it could result from the expression of the mysterious chloroplastic DNA.

It took us over 13 years to reach this result. During this time, molecular biology had taken shape and established the central molecular mechanisms from DNA, ribosomal, messenger, and transfer RNAs, to proteins. We only reached the result in Euglena through biochemical exploration. This was pursued in parallel by scientists who were qualified in genetics and in biochemistry. Had we known this mechanism earlier, we could likely have reached the sought-after result on all species we studied, including the nematodes! Said otherwise, abandoning the nematodes had been a mistake. During this time, it would have been better to acquire the missing experience in genetics and apply it to Rhabditis... If Dougherty and myself had taken up this direction, the scene would have been changed, for both of us. It was the great merit of Brenner: wiser than us, he attacked the nematode only after sufficient experience in Genetics and Molecular Biology!

For those who may be interested in my later works, they are well documented on PubMed, under my name. Only missing are the studies I conducted from 1995 to 1999, on adaptation to high altitude, in La Paz (Bolivia), at 3700 meters. This work was interrupted by the death of my wife and health problems for me, despite some first results possibly oriented towards the discovery of epigenetic processes (still unpublished). 
Text by VM Nigon (translated by by M.-A. Félix).

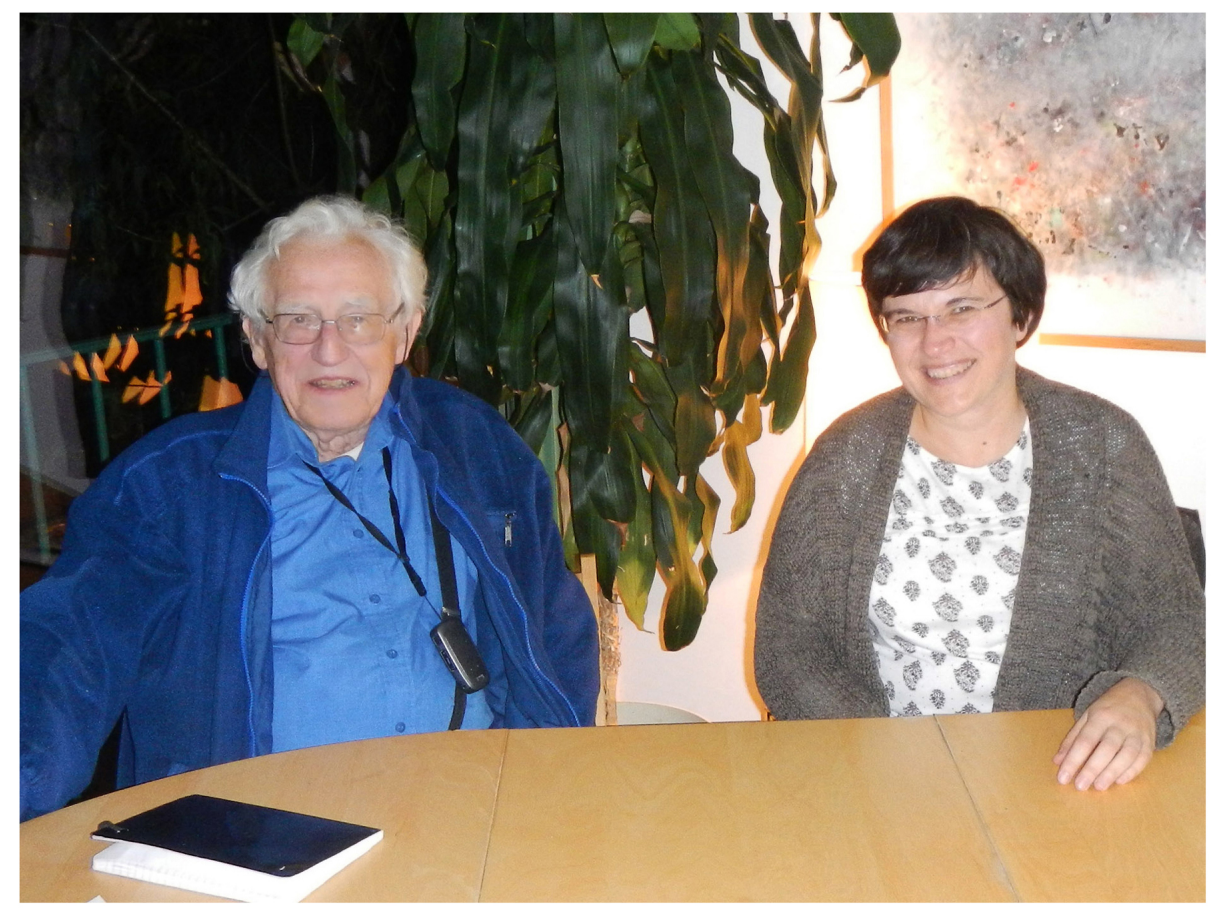

Figure 28. The authors: V.M. Nigon and M.-A. Félix, November 2013.

\subsubsection{Nigon's articles on free-living nematodes}

Delavault, R., and Nigon, V. (1952). L'évolution des acides nucléiques dans les cellules reproductives d'un nématode pseudogame. Arch. Biol. 63, 393-410. Abstract

Dougherty, E.C., and Nigon, V. (1949). A new species of the free-living nematode genus Rhabditis of interest in comparative physiology and genetics. J. Parasitol. 35, 11.

Dougherty, E.C., and Nigon, V. (1953). The effect of acriflavine a mixture of 2,8-diaminoacridine and 2,8-diamino-10-methyl-acridinium-chloride on the growth of the nematode Caenorhabditis elegans. International Congress of Zoology. Copenhagen 14, 247.

Nigon, V. (1943). Le déterminisme du sexe chez un nématode libre hermaphrodite (Rhabditis elegans Maupas). C. R. Soc. Biol. 137, 40-41.

Nigon, V. (1946). Le determinisme du sexe chez un Nematode libre hermaphrodite: Rhabditis dolichura Schneider. Bull. Soc. Zool. France 71, 78-86.

Nigon, V. (1947). Le déterminisme du sexe et la pseudogamie chez un Nématode parthénogénétique, Rhabditis monohystera Bütschli. Bull. Biol. Fr. Belg. 81, 33-37.

Nigon, V. (1949a). Modalités de la reproduction et déterminisme du sexe chez quelques nématodes libres. Ann. des Sc. Nat., Zool. 11, 1-132.

Nigon, V. (1949b). Effets de la polyploïdie chez un nématode libre. C. R. Acad. Sc. Paris D 228, 1161-1162.

Nigon, V. (1951a). Polyploïdie expérimentale chez un nématode libre, Rhabditis elegans Maupas. Bull. Biol. Fr. Belg. 85, 187-225.

Nigon, V. (1951b). Effets d'une élévation de température sur la prophase méiotique d'un Nématode libre. C. R. Acad. Sc. Paris D 232, 1032-1034. 
Nigon, V. (1952a). Modifications expérimentales de la proportion des sexes chez un Nématode pseudogame. C. R. Acad. Sc. Paris D 234, 2568-2570.

Nigon, V. (1952b). L'évolution des acides nucléiques dans les cellules reproductives d'un Nématode pseudogame. Arch. Biol. 63, 393-410.

Nigon, V. (1954). Contributions à la critique expérimentale des théories de la continuité germinale et de la lignée pure. Acta Biol. Acad. Sci. Hungar. 5, 96-117.

Nigon, V. (1965). Développement et reproduction des nématodes. In Traité de Zoologie, P.-P. Grassé, ed. (Paris: Masson).

Nigon, V., and Brun, J. (1955). L'évolution des structures nucléaires dans l'ovogenèse de Caenorhabditis elegans Maupas 1900. Chromosoma 7, 129-169. Abstract

Nigon, V., and Brun, J. (1967). Génétique et évolution des Nématodes libres. Perspectives tirées de l'étude de Caenorhabditis elegans. Experientia 23, 161-240. Abstract

Nigon, V., and Dougherty, E.C. (1949). Reproductive patterns and attempts at reciprocal crossing of Rhabditis elegans Maupas, 1900, and Rhabditis briggsae Dougherty and Nigon, 1949 (Nematoda: Rhabditidae). J. Exp. Zool. 112, 485-503. Abstract

Nigon, V., and Dougherty, E.C. (1950). A dwarf mutation in a nematode; a morphological mutant of Rhabditis briggsae, a free-living soil nematode. J. Hered. 41, 103-109. Abstract Article

Nigon, V., and Dougherty, E.C. (1953). Variations dans la vitesse de croissance chez un Nématode Caenorhabditis elegans (Maupas). Caryologia, Suppl.: Atti Del IX Congresso Internazionale Di Genetica 6, 796-797.

Nigon, V., Guerrier, P., and Monin, H. (1960). L'architecture polaire de l'œuf et les mouvements des constituants cellulaires au cours des premières étapes du développement chez quelques Nématodes. Bull. biol. Fr. Belg. 93, 131-202.

Nigon, V., and Roman, E. (1952). Le déterminisme du sexe et le développement cyclique de Strongyloides ratti. Bull. Biol. Fr. Belg. 86, 405-448. 


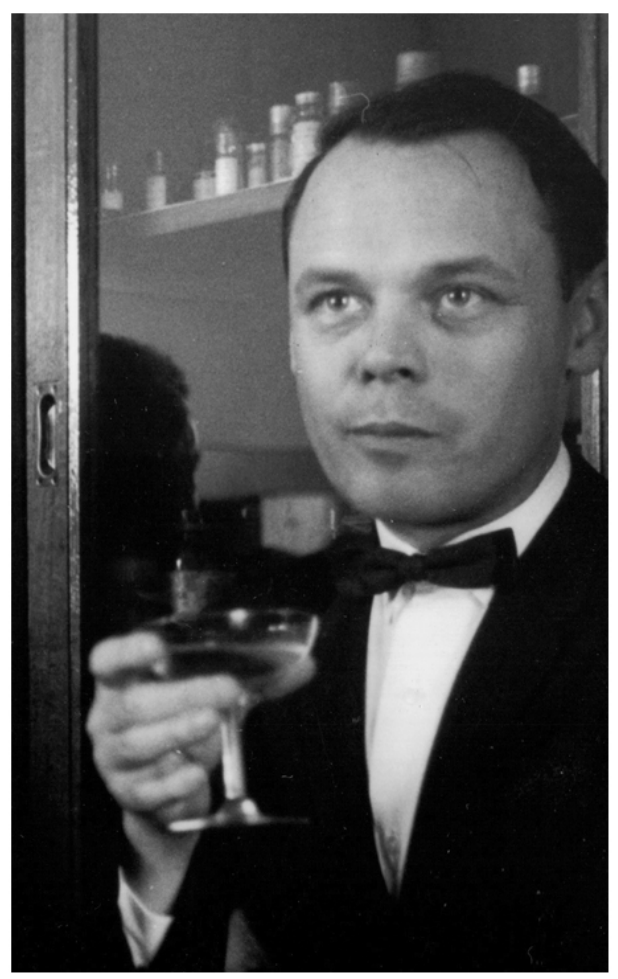

Figure 29. E. C. Dougherty in Lyon, 1961. V.M. Nigon collection.

\subsection{Ellsworth C. Dougherty (1921-1965)}

Ellsworth Dougherty graduated in Zoology at a young age in 1940, and obtained his PhD in 1944 at the University of California in Berkeley. He served in 1944 in the health corps of the US Marines. He then completed his medical studies as a veteran (MD, 1946). As a recipient of a Guggenheim Memorial Fellowship (1947-49), he worked at Caltech and visited France where he stayed in the laboratory of Victor Nigon. After this fellowship, he practiced as a physician in a Kaiser Permanente Foundation hospital in Richmond, CA, and also taught at the University of California in Berkeley.

His first studies concerned systematics of parasitic nematodes. His first work on a free-living nematode, Rhabditis (pellio), took place in Pasadena. He contacted Victor M. Nigon and spent about six months in 1948 working with him in Paris, also attending the International Congress of Zoology that took place in Paris that year. In 1951-52, he came for a second visit to Nigon's laboratory in Lyon for nearly a year. In 1957, he established a laboratory of "Comparative Physiology and Morphology" in the research section of the Kaiser Foundation in Richmond, California. In 1960, he moved to the Department of Nutritional Sciences at the University of California in Berkeley. He joined several scientific trips to Antarctica. He visited Lyon again several times. A last short visit took place in 1961. He had previously suffered from depression that had led him to a first suicide attempt. One of his friends in Lyon, a Professor at the Medical Faculty, organized in his honor an impressive reception. He committed suicide in December 1965.

"We had contracted a deep friendship. Ellsworth had a very imaginative mind and was not naturally an experimenter, yet, by being in contact with me, he got trained and enjoyed the experimental method. In the early 1960s, he hosted in his laboratory Jacqueline Nonnenmacher-Godet, a student from my laboratory (who has served as the head of CNRS Life Sciences in 1999-2002 and is currently President of the French League against Cancer)."-Victor M. Nigon.

E. Dougherty was deeply influenced by George Beadle's results on the biochemical genetics of Neurospora and developed the project to study nutritional mutants, in an animal where the morphology allowed little morphological variation by mutation. He greatly contributed to the nomenclature and development of culture methods of small animals. 
He was a strong advocate of using Rhabditis as model organisms (Dougherty and Calhoun, 1948; Dougherty, 1949). Some of his obvious contributions to present-day C. elegans research are: description of $R$. briggsae with NIgon (Dougherty and Nigon, 1949); axenic cultivation of $R$. briggsae (Dougherty, 1950); raising Caenorhabditis from a subgenus to a genus (Dougherty, 1953); performing the first genetic analysis of $C$. briggsae with Nigon (Nigon and Dougherty, 1950) and of C. elegans Bergerac x N2 with Helene Fatt (Fatt and Dougherty, 1963); and sending N2 to Sydney Brenner (Dougherty, 1963).

\subsubsection{References by Dougherty on nematodes}

Dougherty, E.C. (1949). The rôle of free-living nematodes in genetic research. In Proceedings of the Eighth International Congress of Genetics, 7-14 July 1948, G. Bonnier, R. Larsson, eds. (Lund; Berlingska Boktryckeriet) [Hereditas (suppl)], pp. 520-529. Abstract

Dougherty, E.C. (1950). Sterile pieces of chick embryo as a medium for the indefinite axenic cultivation of Rhabditis briggsae Dougherty and Nigon, 1949 (Nematoda: Rhabditidae). Science 111, 258. Abstract Article

Dougherty, E.C. (1953). The genera of the subfamily Rhabditinae Micoletzky, 1922 (Nematoda). In Thapar Commemoration Volume: A Collection of Articles Presented to Prof. G. S. Thapar on His 60th BIRTHDAY, 1953, J. Dayal, K.S. Singh, eds. (Lucknow, India: University of Lucknow, Department of Zoology), pp. 69-76.

Dougherty, E.C. (1963). Dougherty, Ellsworth C. (Brenner requests C. elegans) C.A. Repository, ed., pp. Reference SB/1/1/162. http://libgallery.cshl.edu/items/show/60761.

Dougherty, E.C., and Calhoun, H.G. (1948a). Possible significance of free-living nematodes in genetic research. Nature 161, 29. Abstract Article

Dougherty, E.C., and Nigon, V. (1949). A new species of the free-living nematode genus Rhabditis of interest in comparative physiology and genetics. J. Parasitol. 35, 11.

Fatt, H.V., and Dougherty, E.C. (1963). Genetic control of differential heat tolerance in two strains of the nematode Caenorhabditis elegans. Science 141, 266-267. Abstract Article

Nigon, V., and Dougherty, E.C. (1950). A dwarf mutation in a nematode; a morphological mutant of Rhabditis briggsae, a free-living soil nematode. J. Hered. 41, 103-109. Abstract Article

See also, Brenner, S. (1974). The genetics of Caenorhabditis elegans. Genetics 77, 71-94. Article

\subsubsection{References on E.C. Dougherty}

Corliss, J.O. (1966). In Memoriam. Trans. Amer. Microscop. Soc. 85, 174. Article

Hansen, T. (1966). Obituary - Ellsworth Charles Dougherty, July 21, 1921 - December 21, 1965. Nematology 12, 470.

Jackson, G.J. (1966). A tribute to Ellsworth Charles Dougherty. Thursday 21, July 1921-Tuesday 21, December 1965. Ann. NY Acad. Sci. 139, 5. Abstract

Ferris, H., and Hieb, W.F. (2015). Ellsworth C. Dougherty: a pioneer in the selection of Caenorhabditis elegans as a model organism. Genetics 200, 991-1002. Abstract Article

\subsubsection{Additional information}

Ellsworth Charles Dougherty, Curriculum Vitae, Department of Nutritional Sciences at the University of California in Berkeley, 25 May 1964. 
18.6. Jean-Louis Brun (1927-1981)

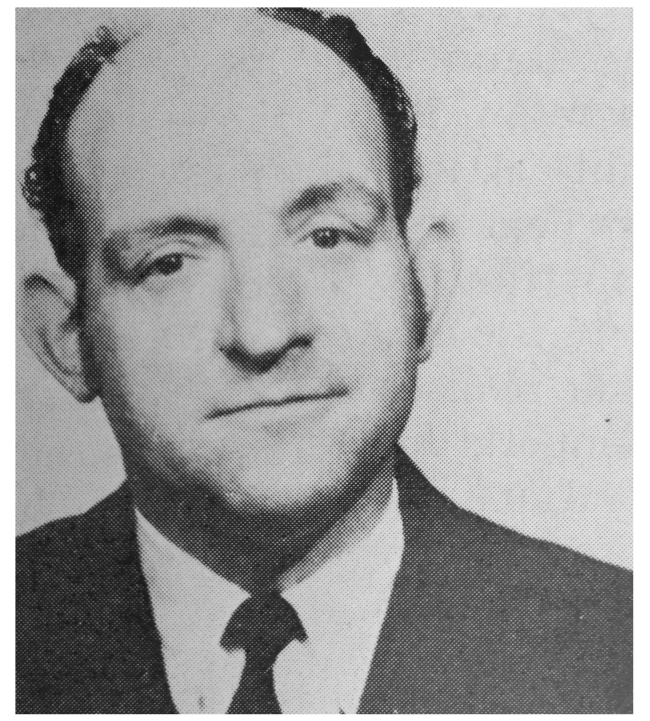

Figure 30. Jean-Louis Brun. From Rev. Nematol. 1982.

Jean-Louis Brun (1927-1981)

Jean Brun's early youth was spent in La Chapelle-de-Mardore, a small village in the Beaujolais region in France. He came from a modest family. His father, a farmer, died from an old World War I wound when Jean Brun and his older brother were young boys. His mother remarried and moved to Lyon, where Jean Brun went to school and was found to excel. Pushed by his teachers, and against his mother's wish that he would work, he continued until the high school degree. Then, needing to work, he combined being a school supervisor in Roanne with studying at the University in Lyon, which he reached through a long bike ride. He met his future wife Geneviève at the university and both joined VM Nigon's laboratory in 1949. While she and others worked for their diplomas on Ascaris, Jean Brun started a project on C. elegans, the species he was to continue with all his life. He obtained an assistant position at the University of Lyon in 1949.

Jean Brun first studied gametogenesis of $C$. elegans, especially chromosome pairing in meiotic prophase. He then described gametogenesis defects at high temperature, including chromosomal abnormalities and cell fusions leading to polyploidy. He experimented with the effects of high temperature on fertility, which led him to the discovery of the progressive decline in fertility over several generations at intermediate temperatures. From there, he patiently adapted the Bergerac strain to $24.5^{\circ} \mathrm{C}$, with $0.5^{\circ} \mathrm{C}$ temperature steps and after hundreds of generations. Through many thorough experiments of temperature reversions, he was led to the conclusion that this adaptation may not be of conventional genetic nature (see main text). Jean Brun was very involved and dedicated to his work. He obtained his "Doctorat d'Etat" in March 1966 with his work on C. elegans temperature adaptation.

Jean Brun and Ellsworth Dougherty were then both interested in developing C. elegans genetics. They had planned for the former to come to Berkeley with his family for the 1966-1967 academic year-a project sadly interrupted by Dougherty's untimely death in December 1965.

From 1967, Jean Brun developed in Lyon an independent research team focused on C. elegans biology and genetics. The team isolated the first mutants of C. elegans (see main text). Jean Brun became an Assistant Professor and became involved in the direction of the academic part of the Biology Department of the University.

The rise of C. elegans through Sydney Brenner, with his scientific aura, was obviously of great importance for the Brun team. They participated in the budding C. elegans scientific community through Worm Breeder Gazettes and the first 1977 Worm Meeting in Woods Hole, MA. Jean Brun and his wife visited David Hirsh in Boulder, CO (in 1976 or 1977). In the later years, Jean Brun developed cancer, yet kept working and following the progress of his team until his death in 1981. Members of the team finished their ongoing studies and published until 1985, then dispersed to other laboratories. 


\subsubsection{A selection on Brun's articles on C. elegans}

Abdul Kader, N., and Brun, J. (1976). Isolation of sterile or lethal temperature-sensitive mutants in Caenorhabditis elegans var. Bergerac. Nematologica 22, 222-226. Article

Abirached, J., and Brun, J.-L. (1978). Ultrastructural changes in the nudear and perinuclear regions of the oogonia and primary oocytes of Caenorhabditis elegans, Bergerac strain. Rev. Nematol. 1, 63-72. Article

Béguet, B., and Brun, J.-L. (1972). Influence of parental aging on the reproduction of the F1 generation in a hermaphrodite nematode Caenorhabditis elegans. Exp. Gerontol. 7, 195-206. Abstract Article

Brun, J. (1955). Evolution de la prophase méiotique chez Caenorhabditis elegans Maupas 1900 sous l'influence des températures élevées. Bull. Biol. Fr. Belg. 89, 326-346.

Brun, J. (1965). Genetic adaptation of Caenorhabditis elegans (Nematoda) to high temperatures. Science 150 , 1467. Abstract

Brun, J.-L. (1966a). L'adaptation aux températures élevées chez un Nématode : Caenorhabditis elegans Maupas 1900. L'adaptation et son évolution. Ann. Biol. Anim. Bioch. Bioph. 6, 127-158. Article

Brun, J.-L. (1966b). L'adaptation aux températures élevées chez un Nématode Caenorhabditis elegans Maupas 1900. II.- Stabilité et physiologie de l'adaptation. Ann. Biol. Anim. Bioch. Bioph. 6, 267-300. Article

Brun, J.-L. (1966c). L'adaptation aux températures élevées chez un Nématode : Caenorhabditis elegans Maupas 1900. III.- Rôle des facteurs autres que la température dans le processus d'adaptation. Ann. Biol. Anim. Bioch. Bioph. 6, 439-466. Article

Dion, M., and Brun, J.L. (1971). Cartographie génique du Nématode libre Caenorhabditis elegans, variété Bergerac. Mol. Gen. Genet. 112, 133-151. Abstract Article

Mounier, N., and Brun, J.-L. (1980). A cytogenetical analysis of sterile mutants in Caenorhabditis elegans. Can. J. Genet. Cytol. 22, 391-403. Abstract

Nigon, V., and Brun, J. (1955). L'évolution des structures nucléaires dans l'ovogenèse de Caenorhabditis elegans Maupas 1900. Chromosoma 7, 129-169. Article

Nigon, V., and Brun, J. (1967). Génétique et évolution des Nématodes libres. Perspectives tirées de l'étude de Caenorhabditis elegans. Experientia 23, 161-240. Article

Ouazana, R., and Brun, J.-L. (1975). Recombinaison intracistronique au niveau d'un locus du nanisme chez le Nématode hermaphrodite Caenorhabditis elegans. C. R. Acad. Sc. Paris D 280, 1895-1898.

Starck, J., Gibert, M.-A., Brun, J., and Bosch, C. (1983). Ribosomal RNA synthesis and processing during oogenesis of the free living nematode Caenorhabditis elegans. Comp. Biochem. Physiol. 75, 575-580. Article

\subsubsection{References on Jean Brun}

Nigon, V.M., and Laviolette, P. (1982). Pr. Jean-Louis Brun 1927-1981. Revue Nematol. 5, 3-5.

All WormBook content, except where otherwise noted, is licensed under a Creative Commons Attribution License. 